\title{
Robust Tests for Deterministic Seasonality and Seasonal Mean Shifts ${ }^{1}$
}

\author{
S. Astill And A.M.R. TAYlor \\ Essex Business School, University of Essex, Wivenhoe Park, Colchester, CO4 3SQ, U.K. \\ E-mail: sastill@essex.ac.uk, rtaylor@essex.ac.uk
}

January 2018

\begin{abstract}
Summary We develop tests for the presence of deterministic seasonal behaviour and seasonal mean shifts in a seasonally observed univariate time series. These tests are designed to be asymptotically robust to the order of integration of the series at both the zero and seasonal frequencies. Motivated by the approach of Hylleberg, Engle, Granger and Yoo [1990, Journal of Econometrics vol. 44, pp. 215-238], we base our approach on linear filters of the data which remove any potential unit roots at the frequencies not associated with the deterministic component(s) under test. Test statistics are constructed using the filtered data such that they have well defined limiting null distributions regardless of whether the data are either integrated or stationary at the frequency associated with the deterministic component(s) under test. In the same manner as Vogelsang [1998, Econometrica vol. 66, pp. 123-148], Bunzel and Vogelsang [2005, Journal of Business and Economic Statistics vol. 23, pp. 381-394] and Sayginsoy and Vogelsang [2011, Econometric Theory vol. 27, pp. 992-1025], we scale these statistics by a function of an auxiliary seasonal unit root statistic. This allows us to construct tests which are asymptotically robust to the order of integration of the data at both the zero and seasonal frequencies. Monte Carlo evidence suggests that our proposed tests have good finite sample size and power properties. An empirical application to U.K. GDP indicates the presence of seasonal mean shifts in the data.
\end{abstract}

Keywords: Seasonality, Seasonal Level Breaks, Seasonal Unit Roots, Robust Tests

\section{INTRODUCTION}

The ability to correctly specify the deterministic component in the econometric analysis of time series processes is crucial for delivering reliable policy modelling, prediction and forecasting. It is also important in the context of unit root testing; in particular, omitting deterministic components present in the underlying data generating process [DGP] can lead to non-similar and inconsistent unit root tests, while the inclusion of irrelevant deterministic components can effect significant efficiency losses, even in large samples.

Perron (1989) showed that an unmodelled broken trend in the DGP can bias standard (zero frequency) unit root tests towards non-rejection of the unit root null, while allowing for an unnecessary broken trend leads to a loss of power to reject the unit root null when the data are stochastically stationary [denoted, following standard convention, $I(0)$ ]. One could therefore envisage pre-testing for the presence of deterministic components prior

\footnotetext{
${ }^{1}$ We thank the Co-Editor, Dennis Kristensen, two anonymous referees, David Harvey, Denise Osborn and Patrick Marsh for helpful and constructive comments. Astill gratefully acknowledges financial support provided by the Economic and Social Research Council of the United Kingdom under research grant ES/I021094/1. Address correspondence to: Robert Taylor, Essex Business School, University of Essex, Wivenhoe Park, Colchester, CO4 3SQ, UK.
} 
to performing a unit root test. This is not straightforward, however. As discussed in Harvey, Leybourne and Taylor (2007), if the data are $I(0)$ then an optimal test for the presence of a linear time trend can be performed on the levels data, whereas if the data admit a zero frequency autoregressive unit root [denoted $I(1)$ ] an optimal test involves testing for a non-zero mean in the first difference of the series. However, tests based on the first differences of the data exhibit poor power properties if the data are, in fact, $I(0)$, and the form of the limiting null distributions of tests based on levels data depend on whether the series is $I(0)$ or $I(1)$. A circular testing problem therefore exists. There has accordingly been a number of papers that look to break this circularity by deriving tests for the presence of deterministic linear and broken linear trend components that are robust to whether the series contains a zero frequency unit root or not; see inter alia Vogelsang (1998), Bunzel and Vogelsang (2005), Harvey, Leybourne and Taylor (2007), Perron and Yabu (2009) and Sayginsoy and Vogelsang (2011).

The assumption that a time series can admit a unit root and deterministic components at only the zero frequency is likely to be too restrictive when we are dealing with seasonally observed data. Here it is natural to allow the deterministic component to vary across the seasons and also to allow autoregressive unit roots to potentially occur at both the zero and seasonal frequencies. Testing for the presence of deterministic components in seasonally observed data is considerably complicated by the fact that the performance of any test using the levels data will depend on the order of integration of the data at all of the zero and seasonal frequencies. Moreover, there is currently no test procedure available to practitioners that allows them to test for the presence of deterministic components in seasonally unadjusted data in such a way as to yield inference which is robust to whether the series contains unit roots at the zero and seasonal frequencies or not. In the absence of such tests, practitioners wishing to perform (zero and/or seasonal frequency) unit root tests on seasonally observed data need to make an ad hoc decision on what form of deterministic seasonality to allow for in their testing procedures, and where an incorrect choice is made qualitatively similar problems to those seen in the non-seasonal case occur; see, for example, Franses and Vogelsang (1998) and Harvey et al. (2002).

One of the most common adjustments made to seasonally observed macroeconomic data is seasonal adjustment. Seasonal adjustment tends to be motivated by the desire to give a clearer picture of the underlying growth rate in the data. The quality and reliability of the resulting seasonally adjusted data are, therefore, heavily dependent on whether the underlying deterministic seasonal component is well specified, or at least well approximated. In relation to this point, the Office for National Statistics [ONS] one of the leading providers of seasonally adjusted data, specifically notes that the quality of its seasonally adjusted data can be negatively impacted by abrupt changes in the seasonal patterns ${ }^{1}$. Even when working with data that is not seasonally adjusted, the presence of deterministic seasonality is of interest in its own right, with deterministic seasonality and any shifts in deterministic seasonality important for both the identification of seasonal effects and the ability to correctly forecast a seasonally observed series; see, in particular, the extensive discussion on these issues in Miron (1996). Clearly, if a time series is subject to structural change in deterministic seasonality, then any forecasts produced from a model that does not account for such a break will be unreliable.

In the non-seasonal context, Vogelsang (1998) and Bunzel and Vogelsang (2005) show that an appropriately constructed test statistic for the presence of zero frequency de-

\footnotetext{
${ }^{1}$ See https://www.ons.gov.uk/methodology/methodologytopicsandstatisticalconcepts/seasonaladjustment
} 
terministic trend components has a limiting distribution which depends on the order of integration of the data at the zero frequency. Based on this result, they apply a scaling factor that is a function of an auxiliary zero frequency unit root test statistic that ensures that, for a given significance level, the asymptotic size of the test procedure is controlled when the data is either integrated or stationary at the zero frequency. Likewise, Sayginsoy and Vogelsang (2011) employ a similar approach to test for breaks in the deterministic component at the zero frequency. We extend the approach of Vogelsang (1998), Bunzel and Vogelsang (2005) and Sayginsoy and Vogelsang (2011) to the seasonal context. Specifically, we propose tests based on data which have been filtered to remove potential unit roots at all but the frequency of the deterministic component(s) under test. We show that appropriate test statistics can be constructed from the filtered data such that they have well defined limiting distributions whose form depends only on the order of integration at the frequency corresponding to the deterministic component(s) under test. Consequently, and paralleling the approach taken in the non-seasonal case, we suggest modifying these statistics by the use of a scaling factor that is a function of an appropriate auxiliary seasonal unit root test statistic. This allows the asymptotic size of the modified tests to be controlled for a given significance level, irrespective of the order of integration of the data at each of the zero and seasonal frequencies.

The remainder of the paper is organised as follows. Section 2 outlines the seasonal model and the underlying assumptions we will work under. Our proposed test statistics for deterministic seasonality and for seasonal mean shifts are outlined in section 3. Section 4 provides asymptotic critical values and scaling constants for implementing the proposed tests and discusses issues relating to optimal bandwidth choice when using kernel based variance estimates. Section 5 discusses issues relating to the practical implementation of the proposed tests. Section 6 reports the results of an empirical application of the proposed tests to seasonally unadjusted quarterly U.K. GDP data. Section 7 concludes. A supplementary paper details the results of a Monte Carlo study into local asymptotic power and finite sample properties of our proposed tests, and provides representations for their limiting distributions under both the null and local alternatives.

\section{THE MODEL AND ASSUMPTIONS}

Consider the univariate process $\left\{x_{t}\right\}$, observed with constant seasonal periodicity, $S$, formed as the sum of a purely deterministic component, $Z_{t}$, and a purely stochastic process, $u_{t}$; viz.,

$$
\begin{aligned}
x_{t} & =Z_{t}+u_{t}, t=1, \ldots, T \\
\alpha(L) u_{t} & =v_{t}, \quad v_{t}=\psi(L) e_{t}
\end{aligned}
$$

where $\alpha(L):=1-\sum_{i=1}^{S} \alpha_{i} L^{i}$ is an $S$ th order autoregressive polynomial in the usual lag operator, $L$. For the purposes of this paper we will concentrate on the quarterly case, $S=4$; generalisations to an arbitrary $S$ are entirely straightforward and only introduce additional notational complexity. Again to simplify notation, but with no loss of generality, we assume that $T=S N$, where $N$ is the number of complete seasonal cycles within the data span. The initial conditions, $u_{-3}, \ldots, u_{0}$, are taken to be of $o_{p}\left(T^{1 / 2}\right)$.

The shocks $v_{t}$ in (2.2) are taken to follow a zero-mean linear process driven by the innovations $e_{t}$. Precise conditions are now detailed in Assumption 2.1.

Assumption 2.1. Let $\left(e_{t}, \mathcal{F}_{t}\right)$ be a martingale difference sequence, with filtration $\mathcal{F}_{t}$, 
where $\mathcal{F}_{t} \subset \mathcal{F}_{t+1}$, for all $t$ and such that: (a) $E\left[e_{t}^{2}\right]=\sigma^{2}$; (b) $1 / T \sum_{t=1}^{T} e_{t}^{2} \stackrel{p}{\rightarrow} \sigma^{2}$, and (c) $E\left|e_{t}\right|^{r}<K$ with $r \geq 4$, where $K$ is some constant depending only upon $r$. The polynomial $\psi(z):=1+\sum_{j=1}^{\infty} \psi_{z} z^{j}$ is such that: (d) $\psi(z) \neq 0$ for all $|z| \leq 1$, and (e) $\sum_{j=1}^{\infty}|j|^{\nu}\left|\psi_{j}\right| \leq \infty$ for some $\nu \geq 1$.

REMARK 2.1. Under Assumption 2.1 the spectral density (and, hence, long run variance) of $v_{t}$ in (2.2) is bounded at both the zero frequency, $\omega_{0}:=0$, and the seasonal spectral frequencies, $\omega_{k}:=2 \pi k / 4, k=1,2$, and is everywhere non-zero. A leading special case of Assumption 2.1 is where $v_{t}$ is a stationary and invertible $A R M A(p, q)$ process.

The polynomial $\alpha(L)$ in $(2.1)$ can be factorised as

$$
\alpha(L)=\prod_{k=0}^{2} \omega_{k}(L)=\left(1-\alpha_{0} L\right)\left(1+2 \beta_{1} L+\left(\alpha_{1}^{2}+\beta_{1}^{2}\right) L^{2}\right)\left(1+\alpha_{2} L\right),
$$

where $\omega_{0}(L):=\left(1-\alpha_{0} L\right)$ associates the parameter $\alpha_{0}$ with the zero frequency $\omega_{0}$, $\omega_{1}(L):=\left(1+2 \beta_{1} L+\left(\alpha_{1}^{2}+\beta_{1}^{2}\right) L^{2}\right)$ corresponds to the harmonic (annual) seasonal frequency and $\omega_{2}(L):=\left(1+\alpha_{2} L\right)$ associates the parameter $\alpha_{2}$ with the Nyquist (biannual) seasonal frequency $\omega_{2}$. We can therefore permit $x_{t}$ to be either (stochastically) stationary or (near-) integrated at the zero and seasonal frequencies through the parameters $\alpha_{k}$, $k=0,1,2$, and $\beta_{1}$. When $\left|\alpha_{i}\right|<1, i=0,2, x_{t}$ is stationary at the zero frequency and the Nyquist frequency, respectively, and when $\alpha_{1}^{2}+\beta_{1}^{2}<1, x_{t}$ is stationary at the annual frequency. Setting $\alpha_{i}=1-\bar{\alpha}_{i} / T, i=0,2$, with $\bar{\alpha}_{i}$ finite constants, $x_{t}$ is (near-) integrated at the zero and Nyquist frequencies, respectively, with $\bar{\alpha}_{i}=0, i=0,2$, yielding an exact unit root at the zero and Nyquist frequencies, respectively. When $\alpha_{1}=1-\bar{\alpha}_{1} / T$, with $\alpha_{1}$ a finite constant, and $\beta_{1}=0$ the process is (near-) integrated at the annual frequency. Here $\bar{\alpha}_{1}=0$ yields a pair of complex conjugate exact unit roots at the annual frequency. As shorthand notation, in what follows we will denote a process which is stationary at frequency $\omega_{k}$ as $I_{\omega_{k}}(0)$, and one which is (near-) integrated at frequency $\omega_{k}$ as $I_{\omega_{k}}(1)$, $k=0,1,2$. In what follows, and where no confusion arises, the terms 'near-integrated' and 'integrated', the latter denoting the exact unit root case, will be used synonymously.

For the purposes of this paper, we will specify the deterministic component $Z_{t}$ in $(2.1)$ using the frequency-based representation,

$$
Z_{t}=z_{t}^{\prime} \gamma+D U_{t, b 0} z_{t}^{\prime} \gamma_{b}
$$

for $z_{t}:=\left(z_{0, t}, z_{1, t}^{\prime}, z_{2, t}\right)^{\prime}$ where $z_{0, t}:=1$, is a (standard) zero frequency intercept, $z_{1, t}:=$ $(\cos [2 \pi t / 4], \sin [2 \pi t / 4])^{\prime}$ is a pair of annual frequency spectral intercepts, and $z_{2, t}:=$ $(-1)^{t}$ is a Nyquist frequency intercept. Moreover, $D U_{t, b 0}$ in (2.4) is a level break dummy variable which takes the value 1 after some (deterministic) break date, $T_{b 0}$. The vectors of associated spectral intercept and spectral level break coefficients are given by $\gamma:=$ $\left(\gamma_{0}, \gamma_{1}^{\prime}, \gamma_{2}\right)^{\prime}$ where $\gamma_{1}=\left(\gamma_{1,1}, \gamma_{1,2}\right)^{\prime}$ and $\gamma_{b}:=\left(\gamma_{0, b}, \gamma_{1, b}^{\prime}, \gamma_{2, b}\right)^{\prime}$ where $\gamma_{1, b}=\left(\gamma_{1,1 b}, \gamma_{1,2 b}\right)^{\prime}$, respectively. The break fraction associated with the latter will be denoted $\lambda_{0}:=T_{b 0} / T$. We assume throughout this paper that the putative break date, $T_{b 0}$, is unknown to the practitioner but that it lies within the set $\Lambda^{*}:=\left\{T_{b}^{*}, T_{b}^{*}+1, \ldots, T-T_{b}^{*}\right\}$, with the convention that $\lambda_{0}$ and $\lambda^{*}:=T_{b}^{*} / T$ remain fixed constants as the sample size increases.

The deterministic component $Z_{t}$ in (2.4) can be equivalently written in terms of standard seasonal indicator variables as,

$$
Z_{t}=d_{t}^{\prime} a+D U_{t, b 0} d_{t}^{\prime} a_{b}
$$


where $d_{t}:=\left(d_{1, t}, d_{2, t}, d_{3, t}, d_{4, t}\right)^{\prime}$, with $d_{i, t}$ a conventional seasonal indicator variable which takes the value 1 if $t$ lies in season $i$ and zero otherwise, $i=1, \ldots, 4$. Defining $a:=\left(a_{1}, \ldots, a_{4}\right)^{\prime}$, the intercept (prior to any seasonal level breaks occurring) in season $i$ is therefore given by $a_{i}, i=1, \ldots, 4$. The vector of break magnitudes, $a_{b}:=\left(a_{b, 1}, \ldots, a_{b, 4}\right)^{\prime}$, entails that the intercept in season $i$ switches from $a_{i}$ to $a_{i}+a_{b, i}$ at time $T_{b 0}$, a break occurring in the level for season $i$ if $a_{b, i} \neq 0, i=1, \ldots, 4$. The representations in (2.5) and (2.4) are mathematically equivalent with $\gamma:=Z_{1}^{*-1} \alpha$ and $\gamma_{b}:=Z_{1}^{*-1} \alpha_{b}$, where the $4 \times 4$ matrix $Z_{1}^{*}:=\left(z_{0}^{* \prime}, z_{1}^{* \prime}, z_{2}^{* \prime}\right)$ and where $z_{i}^{*}:=\left(z_{i, 1}, z_{i, 2}, z_{i, 3}, z_{i, 4}\right), i=0,1,2$. Notice that the columns of the matrix $Z_{1}^{*}$ are mutually orthogonal.

REMARK 2.2. To simplify the outline of the test statistics which follow we have not included either a zero frequency linear trend or a broken zero frequency linear trend in the deterministic component, $Z_{t}$, in (2.4). However, we will discuss in section 5.2 how such components could be dealt with when applying the tests in practice.

REMARK 2.3. Although the seasonal intercepts and seasonal level breaks, $\left\{a_{i}\right\}_{i=1}^{4}$ and $\left\{a_{b, i}\right\}_{i=1}^{4}$ respectively, in (2.5) may be non-zero this does not necessarily mean that there are non-zero spectral means or spectral level shifts at a particular spectral frequency in (2.4). As a simple example, if the magnitude of the seasonal mean shifts average out to zero over a calendar year, such that $\sum_{j=1}^{4} a_{b, j}=0$, then so $\gamma_{0, b}=0$, regardless of the level break magnitudes in each season in (2.5), and, hence, no level break occurs at the zero frequency. More generally, a zero spectral intercept (zero spectral level break) at frequency $\omega_{i}, i=0,1,2$, occurs where $z_{i}^{*}$ and $\alpha\left(z_{i}^{*}\right.$ and $\left.\alpha_{b}\right)$ are orthogonal to each other.

REMARK 2.4. The specification we have adopted for $Z_{t}$ in (2.4) results in the restriction of a common level break date for the deterministic components across all frequencies. While this might appear restrictive, under a more general model where level breaks can occur at different dates at each frequency, the asymptotic theory provided in this paper based on the assumption of a common break date remains valid. This holds because when performing a test at, say, the Nyquist frequency, any level breaks present at other points in the sample at either the zero or annual frequency of the data will manifest in the filtered data as a finite number of impulse dummy terms. These are of asymptotically negligible magnitude and, hence, the omission of these from the deterministic component being modelled has no impact in large samples.

\section{TESTS FOR DETERMINISTIC SEASONALITY AND SEASONAL MEAN SHIFTS}

\subsection{Preliminaries}

Tests for the presence of deterministic seasonality or a shift in deterministic seasonality at a given seasonal frequency (or frequencies), cannot be based on the levels data, $x_{t}$. This is because the form of the limiting null distribution of the resulting test statistics would depend on the order of integration of the data at both the zero and all of the seasonal frequencies. As we shall see, this problem can be circumvented by following an approach first used in Hylleberg et al. (1990) [HEGY, hereafter] and applying test procedures to transformations of the levels data which reduce the order of integration by one at all but the frequency under test. This reduces the problem down to the need to 
develop tests that are robust to whether or not the data are integrated at the particular seasonal frequency (or frequencies) one is testing at which can be readily solved by using a scale factor approach, in the manner of Vogelsang (1998).

To that end, consider the following linear transformations of the levels data:

$$
\begin{aligned}
& x_{0, t}=f_{\omega_{0}}(L) x_{t}:=(1+L)\left(1+L^{2}\right) x_{t}, t=f_{0}+1, . ., T \\
& x_{1, t}=f_{\omega_{1}}(L) x_{t}:=(1-L)(1+L) x_{t}, t=f_{1}+1, . ., T \\
& x_{2, t}=f_{\omega_{2}}(L) x_{t}:=(1-L)\left(1+L^{2}\right) x_{t}, t=f_{2}+1, . ., T .
\end{aligned}
$$

where $f_{i}$ denotes the order of $f_{\omega_{i}}, i=0,1,2$. We will show that the filtered data $x_{2, t}$ can be used for testing hypotheses concerning the Nyquist frequency deterministic component of $Z_{t}$, while $x_{1, t}$ is the relevant filtered data to use for testing hypotheses concerning the annual frequency deterministic component. One could also use the filtered data $x_{0, t}$ to construct tests relating to the deterministic component at the zero frequency. We will not pursue this further here because the limiting distributions of test statistics constructed from $x_{0, t}$ would only depend on the order of integration of $x_{t}$ at the zero frequency so one could, for example, simply apply test procedures of the form proposed in Vogelsang (1998) and Bunzel and Vogelsang (2005) to test for a zero frequency mean, and Sayginsoy and Vogelsang (2011) to test for the presence of a zero frequency mean shift.

We will be able to base our tests on these filtered data because the transformations $f_{\omega_{i}}(L) x_{t}, i=1,2$, reduce the order of integration by one at each frequency $\omega_{k}, k \neq i$. From (2.1)-(2.2), for $i=1,2$, we have that $f_{\omega_{i}}(L) x_{t}=f_{\omega_{i}}(L) Z_{t}+u_{i, t}, t=f_{i}+1, \ldots, T$, where $u_{i, t}:=f_{\omega_{i}}(L) u_{t}$. Consequently, $\omega_{i}(L) u_{i, t}=v_{i, t}$, where $v_{i, t}:=f_{\omega_{i}}(L) \prod_{k \neq i} \omega_{k}(L)^{-1} v_{t}$, $i=1,2$. The filtered error process $v_{i, t}, i=1,2$, might therefore contain moving average unit roots (equivalently spectral zeroes) but only at the frequencies at which the order of integration has been reduced. For example, the filtered data appropriate for testing hypotheses concerning the Nyquist frequency deterministic component, $x_{2, t}$, would contain moving average unit roots in $v_{2, t}$ at the zero $\left(\omega_{0}=0\right)$ frequency if $x_{t}$ was $I_{0}(0)$, and at the annual $\left(\omega_{1}=\pi / 2\right)$ frequency if $x_{t}$ was $I_{\pi / 2}(0)$, but would not contain moving average unit roots in $v_{2, t}$ at the Nyquist $\left(\omega_{2}=\pi\right)$ frequency.

It is also important to notice that the filtered series $x_{i, t}, i=1,2$, will only contain deterministic components associated with frequency $\omega_{i}$. However, where a level break occurs the filtered series $x_{i, t}, i=1,2$, will also contain $f_{i}$ additional impulse dummy terms occurring at the dates $t=T_{b 0}+1, \ldots, T_{b 0}+f_{i}$ immediately following the level shift. Specifically, the filtered series $x_{i, t}, i=1,2$, satisfy

$$
x_{i, t}=F_{i, m}(t)^{\prime} \delta_{\omega_{i}, m}+F_{i, m s}\left(t, \lambda_{0}\right)^{\prime} \delta_{\omega_{i}, m s}+\sum_{j=1}^{f_{i}} \eta_{j} I_{T_{b 0}+j}+u_{i, t}
$$

where $I_{T_{b 0}+j}$, is an impulse dummy variable that takes the value 1 if $t=T_{b 0}+j$ and 0 otherwise, and where $F_{i, m}(t):=z_{i, t}$ is the vector formed of those regressors which do not depend on the break date and $F_{i, m s}\left(t, \lambda_{0}\right)=z_{i, t} D U_{t, b 0}$ is the vector formed of those regressors (other than the impulse dummies) whose form depends on the break date. In (3.4), $\delta_{\omega_{i}, m}:=k \gamma_{i}$ and $\delta_{\omega_{i}, m s}:=k \gamma_{i, b}$, where $k=2$ when $i=1$ and $k=4$ when $i=2$; that is, the filter applied to the data magnifies the coefficient on any deterministic components at the frequency under test. The coefficients on the impulse dummies, are functions of the break magnitudes on any level breaks present at the zero and all of the seasonal frequencies. They are therefore uninformative in practice about the break magnitude(s) at any proper subset of the zero and seasonal frequencies. 
In what follows we will consider two different classes of tests. The first, outlined in section 3.2 focuses on testing whether deterministic seasonality is present at frequency $\omega_{i}, i=1,2$. We do so by considering the filtered data $x_{i, t}$ and testing for the presence of the deterministic components contained in $F_{i, m}(t)$ in (3.4). These tests will therefore be based on estimating the following regression by OLS

$$
x_{i, t}=\sum_{\substack{j=0 \\ j \neq i}}^{2} z_{j, t}^{\prime} \tilde{\gamma}_{j}^{*}+F_{i, m}(t)^{\prime} \tilde{\delta}_{\omega_{i}, m}+\tilde{u}_{i, t}
$$

setting $i=1$ and $i=2$ for the annual $\left(\omega_{1}\right)$ and Nyquist $\left(\omega_{2}\right)$ frequencies, respectively.

The second class of tests we consider, outlined in section 3.3, test whether a level break is present at frequency $\omega_{i}, i=1,2$, by testing for the presence of the deterministic components contained in $F_{i, m s}\left(t, \lambda_{0}\right)$ in (3.4). For a generic putative break date, $T_{b}$, and associated break fraction, $\lambda:=T_{b} / T$, these would based on the estimated OLS regression

$$
x_{i, t}=\sum_{\substack{j=0 \\ j \neq i}}^{2} z_{j, t}^{\prime} \hat{\gamma}_{j}^{*}+F_{i, m}(t)^{\prime} \hat{\delta}_{\omega_{i}, m}+F_{i, m s}(t, \lambda)^{\prime} \hat{\delta}_{\omega_{i}, m s}+\sum_{j=1}^{f_{i}} \hat{\eta}_{j} I_{T_{b}+j}+\hat{u}_{i, t} .
$$

again setting $i=1$ for tests at the annual frequency and $i=2$ for tests at the Nyquist frequency. However, because the true break date $T_{b 0}$ is assumed unknown, we will construct estimates of the form in (3.6) over all possible break dates $T_{b}$ within the set $\Lambda^{*}{ }^{2}$

\subsection{Tests for Seasonal Spectral Means}

We first consider tests of the separate null hypotheses $H_{0}^{\omega_{1}, m}: \delta_{\omega_{1}, m}=0$ and $H_{0}^{\omega_{2}, m}$ : $\delta_{\omega_{2}, m}=0$ in (3.4) (equivalently, $\gamma_{1}=0$ and $\gamma_{2}=0$, respectively, in (2.4)). In each case we will work under the maintained hypothesis that no seasonal level break is present in $Z_{t}$; that is, $\delta_{\omega_{1}, m s}=\delta_{\omega_{2}, m s}=0$ (equivalently, that $\gamma_{1, b}=\gamma_{2, b}=0$ in (2.4)). However, it should be clear that such tests would also be consistent against series which display level breaks at the seasonal frequency under test, and indeed many other more general deterministic seasonal patterns at that frequency. The first of these null hypotheses involves two linear restrictions (other than those imposed by the maintained hypothesis) that together entail there being no deterministic seasonal component present at the annual frequency. The second involves one linear restriction which entails there being no deterministic seasonal component present at the Nyquist frequency. Tests of the joint null hypothesis of no deterministic seasonal component, such that $\gamma_{1}=\gamma_{2}=0$ in (2.4), will be subsequently discussed in section 3.5.

Following the approach taken for the non-seasonal case in Bunzel and Vogelsang (2005), we will test the null hypotheses $H_{0}^{\omega_{i}, m}, i=1,2$, using the heteroskedasticity autocorrelation (HAC) robust Wald-type statistics

$$
W_{\omega_{i}, m, B V}:=\tilde{\delta}_{\omega_{i}, m}^{\prime}\left[\tilde{\sigma}_{B V, \omega_{i}}^{2}\left[\sum_{t=f_{i}+1}^{T} F_{i, m}(t) F_{i, m}(t)^{\prime}\right]^{-1}\right]^{-1} \tilde{\delta}_{\omega_{i}, m}
$$

\footnotetext{
${ }^{2}$ We include the regressors $z_{j, t}, j \neq i$, in (3.5) and (3.6) to ensure that the statistics we develop for testing hypotheses on $\gamma_{i}, i \in\{1,2\}$, are exact invariant to the $\gamma_{j}, j=0,1,2, j \neq i$, parameters in (2.4).
} 
where $\tilde{u}_{i, t}$ and $\tilde{\delta}_{\omega_{i}, m}$ are the OLS residuals and OLS estimator of $\delta_{\omega_{i}, m}$, respectively, from (3.5), and where

$$
\tilde{\sigma}_{B V, \omega_{i}}^{2}:=\tilde{\gamma}_{0, \omega_{i}}+2 \sum_{j=1}^{T-f_{i}-1} k(j / M) \tilde{\gamma}_{j, \omega_{i}}
$$

is a spectral long run variance estimator where $\tilde{\gamma}_{j, \omega_{i}}:=T^{-1} \sum_{t=j+f_{i}+1}^{T}\left(z_{i, t} \tilde{u}_{i, t}\right)\left(z_{i, t} \tilde{u}_{i, t-j}\right)^{\prime}$ are the sample autocovariances. We make the following assumptions on the kernel, $k(x)$ and bandwidth, $M$.

Assumption 3.1. The kernel function $k(x)$ is continuous at $x=0$ and satisfies $k(x)=$ $k(-x), k(0)=1,|k(x)| \leq 1$ and $\int_{0}^{1} k(x)^{2} d x<\infty . k(x)$ is also twice continuously differentiable everywhere with associated second derivative $k^{\prime \prime}(x)$. We also define $k^{*}(x)=k(x / b)$ where the bandwidth $M$ is such that $M:=\lfloor b T\rfloor,\lfloor\cdot\rfloor$ denoting the integer part of its argument, with bandwidth fraction $b \in(0,1]$.

REMARK 3.1. The kernel function $k(x)$ is therefore assumed to satisfy the conditions given for a type 1 kernel in Sayginsoy and Vogelsang (2011). Like Sayginsoy and Vogelsang (2011), we found that amongst a range of popularly applied kernel functions, tests based on the Daniell kernel delivered the best finite sample performance. As a result, all of the numerical results reported in this paper pertain to the use of the Daniell kernel.

For each of $i=1,2$, an alternative and closely related test statistic can be formed by replacing the HAC estimator $\tilde{\sigma}_{B V, \omega_{i}}^{2}$ in (3.7) with $\tilde{\sigma}_{V, \omega_{i}}^{2}$, where $\tilde{\sigma}_{V, \omega_{i}}^{2}$ is a seasonal variance estimator, corresponding to frequency $\omega_{i}$, based on the spectrally (at frequency $\omega_{i}$ ) cumulated OLS residuals $\tilde{u}_{i, t}$ from (3.5),

$$
\tilde{\sigma}_{V, \omega_{i}}^{2}:=T^{-2} \sum_{t=f_{i}+1}^{T}\left(\sum_{s=f_{i}+1}^{t}\left(z_{i, s} \tilde{u}_{i, s}\right) \sum_{s=f_{i}+1}^{t}\left(z_{i, s} \tilde{u}_{i, s}\right)^{\prime}\right) .
$$

The resulting statistics, which we denote by $W_{\omega_{i}, m, V}, i=1,2$, can be viewed as the seasonal frequency analogues (aside from the scale factor which we will subsequently discuss in section 3.4 below) of the PSW test statistic of Vogelsang (1998) for the present testing problem. ${ }^{3}$ Notice that in Vogelsang (1998) because the hypotheses being tested relate to a zero frequency deterministic component and robustness is required to the order of integration of the data at the zero frequency, the equivalent of $\tilde{\sigma}_{V, \omega_{i}}^{2}$ would be calculated from standard (rather than spectrally) cumulated residuals; that is, standard partial sums of the residuals would taken, not the seasonal partial sums required here.

\subsection{Tests for Seasonal Spectral Mean Shifts}

We next develop tests of the null hypotheses $H_{0}^{\omega_{1}, m s}: \delta_{\omega_{1}, m s}=0$ and $H_{0}^{\omega_{2}, m s}: \delta_{\omega_{2}, m s}=0$ in (3.4). The former involves two linear restrictions, and entails that there is no seasonal

\footnotetext{
${ }^{3}$ Notice that we spectrally partially sum the residuals from regression (3.5) rather than use residuals from a spectral partial sum counterpart of regression (3.5) which would be the exact analogue of the tests of Vogelsang (1998). It can be shown, however, that both methods of calculating the variance estimate lead to tests with identical asymptotic size and local asymptotic power. We choose the former method as it involves estimating one fewer regression, thereby saving computational time.
} 
level break present at the annual frequency, $\omega_{1}$. The latter imposes one linear restriction that entails there being no level break in the seasonal component at the Nyquist frequency, $\omega_{2} \cdot{ }^{4}$ Joint frequency tests will again be discussed in section 3.5.

If the true break date, $T_{b 0}$, were known then $H_{0}^{\omega_{i}, m s}, i=1,2$, could be tested along the same lines considered for the tests for deterministic seasonality in section 3.2 by using the HAC robust Wald statistics:

$$
W_{\omega_{i}, m s, B V}\left(T_{b 0}\right):=\hat{\delta}_{\omega_{i}, m s}^{\prime}\left[\hat{\sigma}_{B V, \omega_{i}}^{2} R_{i}\left[\sum_{t=f_{i}+1}^{T} X_{i, t, \lambda_{0}}^{\prime} X_{i, t, \lambda_{0}}\right]^{-1} R_{i}^{\prime}\right]^{-1} \hat{\delta}_{\omega_{i}, m s}
$$

where $R_{1}:=\left[\begin{array}{ccccccc}0 & 0 & 1 & 0 & 0 & \cdots & 0 \\ 0 & 0 & 0 & 1 & 0 & \cdots & 0\end{array}\right], R_{2}:=(0,1,0, \ldots, 0)$, and, in each case for $i=1,2, \hat{\delta}_{\omega_{i}, m s}$ is the OLS estimate of $\delta_{\omega_{i}, m s}$ from $(3.6)$ and $X_{i, t, \lambda_{0}}:=\left[F_{i, m}(t)^{\prime}, F_{i, m s}\left(t, \lambda_{0}\right)^{\prime}, I_{T_{b 0}+1}, \ldots, I_{T_{b 0}+f_{i}}\right]$. The HAC variance estimator $\hat{\sigma}_{B V, \omega_{i}}^{2}$ is constructed exactly as for the $W_{\omega_{i}, m, B V}$ statistic in section 3.2 but using the OLS residuals, $\hat{u}_{i, t}$, from estimating (3.6) rather than $\tilde{u}_{i, t}$. Again, an alternative statistic can be formed using the OLS-type variance estimator of the form given in (3.9) but again with $\hat{u}_{i, t}$ replacing $\tilde{u}_{i, t}$; we will denote this estimator by $\hat{\sigma}_{V, \omega_{i}}^{2}$ and the resulting test statistic by $W_{\omega_{i}, m s, V}$.

In the case we are concerned with in this paper where the true break date, $T_{b 0}$, is taken to be unknown to the practitioner, we follow the approach of Sayginsoy and Vogelsang (2011) and base our tests on the supremum of the sequences of Wald statistics of the form given in (3.10) evaluated across all possible break dates $T_{b}$ in the search set $\Lambda^{*}=$ $\left\{T_{b}^{*}, T_{b}^{*}+1, \ldots, T-T_{b}^{*}\right\}$. Our proposed test statistics for testing for a seasonal mean shift at frequency $\omega_{i}, i=1,2$ (where we again recall that $i=1$ corresponds to the annual frequency, $\omega_{1}$, and $i=2$ to the Nyquist frequency, $\left.\omega_{2}\right)$, are therefore given by

$$
S u p W_{\omega_{i}, m s, B V}:=\sup _{T_{b} \in \Lambda^{*}} W_{\omega_{i}, m s, B V}\left(T_{b}\right) .
$$

REMARK 3.2. In addition to the supremum-based statistics in (3.11), we also considered the corresponding statistics based on taking the average across the sequence of Wald statistics, $\left\{W_{\omega_{i}, m s, B V}\left(T_{b}\right)\right\}_{T_{b} \in \Lambda^{*}}$. However, and in accordance with the findings in Sayginsoy and Vogelsang (2011) for the problem of testing for a zero frequency mean shift, we found that tests based on supremum-based statistic delivered superior power properties to the corresponding average-based tests. We therefore only report results for tests based on the supremum statistic in what follows. Corresponding results for tests based on the average statistic can be obtained from the authors on request.

\subsection{Scaled Statistics}

Although, as the results presented in section 4 will show, the Wald-type statistics proposed in sections 3.2 and 3.3 have well defined limiting null distributions, both when the data are stationary and when the data are integrated at the frequency under test, crucially these limiting distributions do not coincide. In particular, for a given statistic,

\footnotetext{
${ }^{4}$ It is important to note here that, in the light of Remarks 2.3 and 2.4 and the discussion following (3.4), it would not be appropriate to also include zero restrictions on the $\eta_{j}, j=1, \ldots, f_{i}$, coefficients in (3.6) when testing for level shifts because these coefficients could be non-zero in cases where a level break is not present at frequency $\omega_{i}$. but a level break is present at frequency $w_{j} j \neq i$.
} 
the limiting null distribution in the integrated case is right-skewed relative to the corresponding limiting null distribution in the stationary case. Therefore, and in the same manner as is done in, inter alia Vogelsang (1998) and Bunzel and Vogelsang (2005), and Sayginsoy and Vogelsang (2011), we propose scaling the aforementioned Wald-type statistics for seasonal means and seasonal mean shifts by an exponential function of an auxiliary seasonal unit root test statistic such that, for a chosen significance level, the tests have asymptotically controlled size regardless of whether the data are stationary or integrated at the seasonal frequency under test (as well as to the order of integration of the data at the other spectral frequencies).

We use $W_{\omega_{i}, m}$ to generically denote either $W_{\omega_{i}, m, B V}$ or $W_{\omega_{i}, m, V}$ and $S u p W_{\omega_{i}, m s}$ to generically denote either $S u p W_{\omega_{i}, m s, B V}$ or $S u p W_{\omega_{i}, m s, V}$, in each case for $i=1,2$ (recall that $i=1$ relates to the annual frequency, $\omega_{1}=\pi / 2$, and $i=2$ to the Nyquist frequency, $\omega_{2}=\pi$ ). We will also use the notation $U R$ to generically denote the unit root statistic used in the scale factor approach. The scaled statistics can, thus, be written in corresponding generic form as:

$$
\begin{aligned}
W_{\omega_{i}, m}(U R) & :=W_{\omega_{i}, m} \exp \left(-c_{\omega_{i}, m}^{U R} U R_{\omega_{i}, m}\right) \\
S u p W_{\omega_{i}, m s}(U R) & :=\operatorname{Sup} W_{\omega_{i}, m s} \exp \left(-c_{\omega_{i}, m s}^{U R} U R_{\omega_{i}, m s}\right) .
\end{aligned}
$$

In the context of (3.12) and (3.13), $c_{\omega_{i}, m}^{U R}$ and $c_{\omega_{i}, m s}^{U R}$ are scaling constants while $U R_{\omega_{i}, m}$ and $U R_{\omega_{i}, m s}$ are seasonal unit root test statistics that respectively allow for either spectral means or spectral means and spectral mean shifts in their modelled deterministic component. These unit root statistics will need to possess the properties that where $x_{t}$ is $I_{\omega_{i}}(0)$ (i.e. stationary at the frequency under test) they converge to zero, and where $x_{t}$ is $I_{\omega_{i}}(1)$ (i.e. integrated at the frequency under test) they have a well-defined limiting distribution that does not depend on any unknown nuisance parameters, other than $\bar{\alpha}_{i}$, under the null hypothesis being tested. As a result, where $x_{t}$ is $I_{\omega_{i}}(0)$ the exponential scaling factors in (3.12) and (3.13) will converge to unity as the sample size diverges leaving the asymptotic distribution of the statistics unaffected. In the case where $x_{t}$ is $I_{\omega_{i}}(1)$, and selecting the $\xi$ level critical value from the asymptotic null distribution of $W_{\omega_{i}, m}$ or $\operatorname{Sup} W_{\omega_{i}, m s}$ appropriate for the stationary case, we can, therefore, for any candidate seasonal unit root test statistic, choose values of the scaling constants $c_{\omega_{i}, m}^{U R}$ or $c_{\omega_{i}, m s}^{U R}$ such that the asymptotic sizes of the tests based on the resulting scaled statistics and this critical value do not exceed $\xi \%$ across a range of values of $\bar{\alpha}_{i}$.

We consider three possible sets of candidate seasonal unit root statistics to use in the context of the scaling factors in (3.12) and (3.13). Consider first the spectral mean testing case in (3.12). The first set is motivated by the choice of statistic made in Vogelsang (1998) and is therefore based on a generalisation of the zero frequency unit root statistics of Park (1990) and Park and Choi (1988) to the seasonal unit root testing context. To that end, we first estimate the following regression by OLS

$$
x_{i, t}=\gamma_{0} z_{0, t}+\gamma_{1}^{\prime} z_{1, t}+\gamma_{2} z_{2, t}+\sum_{j=1}^{m} \zeta_{j}\left(z_{i, t} t^{j}\right)+e_{t} .
$$

We then construct the statistics $J_{\omega_{i}}:=\left(S S R_{i, 1}-S S R_{i, 2}\right) /\left(S S R_{i, 2}\right)$, where $S S R_{i, 1}$ denotes the sum of squared residuals from estimating (3.14) and $S S R_{i, 2}$ denotes the sum of squared residuals from the OLS estimation of $x_{i, t}$ onto $z_{0, t}, z_{1, t}$ and $z_{2, t}$. We set $m=9$ for the Nyquist frequency unit root test statistic, $J_{\omega_{2}}$, and $m=8$ for the annual frequency unit root test statistic, $J_{\omega_{1}}$, as we found these choices of $m$ gave the best asymptotic 
power performance for the resulting tests for seasonal means at the Nyquist and annual frequencies, respectively. The scaled statistics in (3.12) based on $J_{\omega_{i}}$ will be denoted $W_{\omega_{i}, m}(J)$ with the associated scaling constant denoted as $c_{\omega_{i}, m}^{J}, i=1,2$ in what follows.

The second set of unit root statistics to use in (3.12) are the seasonal variance ratio unit root test statistics proposed in Taylor (2005). For the Nyquist frequency, this is given by

$$
V R_{\omega_{2}, m}:=T^{-3} \tilde{\Gamma}_{2}^{-1} \sum_{t=4}^{T}\left(\sum_{j=4}^{t} z_{2, j} \tilde{u}_{2, j}\right)^{2}
$$

while the variance ratio statistic for the annual frequency is given by

$$
V R_{\omega_{1}, m}:=T^{-3} \operatorname{trace}\left[\tilde{\Gamma}_{1}^{-1} \sum_{t=3}^{T}\left(\sum_{j=3}^{t} z_{1, j} \tilde{u}_{1, j}\right) \sum_{t=3}^{T}\left(\sum_{j=3}^{t} z_{1, j} \tilde{u}_{1, j}\right)^{\prime}\right]
$$

$\tilde{u}_{i, t}, i=1,2$ the OLS residuals from (3.5) and where $\tilde{\Gamma}_{i}:=T^{-1} \sum_{t=f_{i}+1}^{T}\left(\tilde{u}_{i, t} z_{i, t}\right)\left(\tilde{u}_{i, t} z_{i, t}\right)^{\prime}$. The scaled statistics in (3.12) based on $V R_{\omega_{i}, m}$ will be denoted $W_{\omega_{i}, m}(V R)$ with the associated scaling constant denoted as $c_{\omega_{i}, m}^{V R}, i=1,2$ in what follows.

Harvey, Leybourne and Taylor (2006) modify the zero frequency linear trend test of Vogelsang (1998) by constructing the scaling factor using the reciprocal of the absolute value of a standard augmented Dickey-Fuller statistic. They show that this can improve the finite sample properties of the resulting trend test relative to the use of the Park and Choi (1988) statistic. In the light of these findings, the final set of candidate seasonal unit root statistics we will consider for use in (3.12) are based on the HEGY regression-based seasonal unit root statistics. These are obtained from OLS estimation of the regression

$$
\begin{aligned}
\Delta_{4} x_{t}= & d_{t}^{\prime} \alpha+\pi_{0} x_{0, t-1}+\pi_{2}\left(-x_{2, t-1}\right)+\pi_{3}\left(-x_{1, t-2}\right)+\pi_{4}\left(-x_{1, t-1}\right) \\
& +\sum_{j=1}^{q} \phi_{j} \Delta_{4} x_{t-j}+u_{t}, \quad t=q+5, \ldots, T,
\end{aligned}
$$

where $\Delta_{4}:=\left(1-L^{4}\right)$, and the lag length, $q$, satisfies the usual rate condition that $1 / q+q^{3} / T \rightarrow 0$ as $T \rightarrow \infty$. The HEGY test statistic for a Nyquist frequency unit root is given by the standard regression $t$-ratio for $\pi_{2}=0$, say $t_{2}$, in (3.17) and that for the (complex pair of) annual frequency unit roots is given by the regression $F$-statistic for $\pi_{3}=\pi_{4}=0$ in $(3.17)$, say $F_{34}$. In each case our candidate unit root statistic will be based on the reciprocal of the absolute value of the statistic. The scaled statistics in (3.12) using the appropriate frequency HEGY statistic will be denoted $W_{\omega_{i}, m}(H)$ with the associated scaling constant denoted as $c_{\omega_{i}, m}^{H}, i=1,2$ in what follows.

Consider next the scaling factor used in connection with the seasonal spectral level shift tests in (3.13). The seasonal unit root statistics outlined above can be straightforwardly adapted to the case where a level break may occur at a known break date $T_{b 0}$. For the $J_{\omega_{2}}$ and $J_{\omega_{2}}$ statistics one simply augments the test regression in (3.14) with the additional regressors, $z_{k, t} D U_{t, b 0}, k=0,1,2$ and $I_{T_{b 0}+j} j=1, \ldots, f_{i}$. For tests based on HEGY statistics, we may use the reciprocals of HEGY-type test statistics obtained from the two-step procedure embodied in equations (4)-(6) of Franses and Vogelsang (1998), whereby the broken deterministic components of $x_{t}$ are first estimated, and a HEGY regression, using appropriate dummy variables, is applied to the residuals from this firststep regression. For tests based on the variance ratio statistics, these would be computed 
as in (3.15) and (3.16) but now using the OLS residuals $\hat{u}_{2, t}$ and $\hat{u}_{1, t}$, respectively, from (3.6); we denote the resulting statistics as $V R_{\omega_{i}, m s}\left(T_{b 0}\right), i=1,2$.

In practice the putative trend break date is unknown, and here we follow the approach of Sayginsoy and Vogelsang (2011). To that end, for each frequency we will calculate a unit root test statistic that allows for spectral mean shifts described above for a generic possible break date, $T_{b}$, and do so across all values of $T_{b} \in \Lambda^{*}$. As we treat the true break date as unknown we then select the infimum of unit root statistics in the resulting sequence following Sayginsoy and Vogelsang (2011). Specifically, denoting a generic unit root test statistic at frequency $\omega_{i}, i=1,2$, which allows for a level break at time $T_{b}$ as $U R_{\omega_{i}, m s}\left(T_{b}\right)$, we would calculate $U R_{\omega_{i}, m s}^{*}:=i n f_{T_{b} \in \Lambda^{*}} U R_{\omega_{i}, m s}\left(T_{b}\right)$, for $i=1,2$. In what follows we will consider level break tests based on the infimum of the sequence of $V R_{\omega_{i}, m s}\left(T_{b}\right)$ statistics when testing for a seasonal mean shift at frequency $\omega_{i}$; that is,

$$
V R_{\omega_{i}, m s}^{*}:=\inf _{T_{b} \in \Lambda^{*}} V R_{\omega_{i}, m s}\left(T_{b}\right), \quad i=1,2 .
$$

The scaled statistics in (3.13) based on $V R_{\omega_{i}, m s}^{*}$ will be denoted $S u p W_{\omega_{i}, m s}(V R)$ with the associated scaling constant denoted as $c_{\omega_{i}, m s}^{V R}, i=1,2$ in what follows. Tests based on the Park and Choi (1989)-type statistics and the HEGY-type statistics were found to deliver very poor finite sample behaviour and so will not be discussed further here.

\subsection{Joint Frequency Tests}

The test procedures outlined thus far are designed to test for a seasonal spectral mean or a shift in seasonal spectral mean at either the annual frequency, $\omega_{1}=\pi / 2$, or the Nyquist frequency, $\omega_{2}=\pi$. In practice, one might wish to also consider joint tests for the presence of seasonal spectral means or level shifts in seasonal spectral means occurring at either or both frequencies, thereby yielding size controlled tests for deterministic seasonality in the former case, and for a shift in deterministic seasonality in the latter case.

Joint tests are straightforward to develop because the limiting distributions of the test statistics constructed at frequency $\omega_{i}$ will turn out to be independent of the limit distributions of the statistics constructed at frequency $\omega_{j} i, j=1,2, j \neq i$. Simulation results in the supplementary paper show that the best overall performance when testing for individual frequency spectral means and spectral mean shifts are obtained using the $W_{\omega_{i}, m, B V}(V R)$ and the $S u p W_{\omega_{i}, m s, B V}(V R)$ statistics, respectively, and so we will focus attention in what follows on how joint tests for spectral means and spectral mean shifts can be constructed from these individual frequency statistics; however, the same principles could be applied to any of the individual frequency statistics discussed previously.

A natural basis to use for developing a joint test for the presence of seasonal means is the simple average ${ }^{5}$ of modified versions of the individual annual and Nyquist frequency statistics, $W_{\omega_{1}, m, B V}(V R)$ and $W_{\omega_{2}, m, B V}(V R)$ respectively; that is,

$$
J S_{\delta_{m}}(V R):=\frac{1}{2} \sum_{i=1}^{2} W_{\omega_{i}, m} \exp \left(-\tau_{\delta_{m}} c_{\omega_{i}, m, B V}^{V R} V R_{\omega_{i}, m}\right)
$$

where $c_{\omega_{i}, m, B V}^{V R} i=1,2$ are the scaling constants appropriate for the individual frequency $W_{\omega_{1}, m, B V}(V R)$ and $W_{\omega_{2}, m, B V}(V R)$ tests $i=1,2$ that will be detailed in Section 4.1.

5 This gives equal weight to each of the individual frequency statistics. Tests based on a weighted average might also be considered but we will not do so here. 
Note that if we took a simple average of the two individual frequency $W_{\omega_{1}, m, B V}(V R)$ and $W_{\omega_{2}, m, B V}(V R)$ test statistics there would be many possible combinations of $c_{\omega_{i}, m, B V}^{V R}$ $i=1,2$ that would yield a robust joint test. As such, the appropriate scaling constants for the individual frequency tests are both multiplied by a further scaling constant $\tau_{\delta_{m}}$, where $\tau_{\delta_{m}}$ can be chosen such that the asymptotic size of the resulting test does not exceed $\xi \%$ when the data is $I_{\omega_{i}}(0)$ or $I_{\omega_{i}}(1), i=1,2$. This has the practical advantage of reducing the problem from choosing between multiple pairs of $c_{\omega_{i}, m, B V}^{V R}, i=1,2$, to selecting a single value of $\tau_{\delta_{m}}$.

When testing for the presence of a shift in deterministic seasonality we can form a joint test across both frequencies in the same manner. Specifically, a test for a break in deterministic seasonality at either or both of the annual and Nyquist frequencies can be constructed as

$$
J S_{\delta_{m s}}(V R):=\frac{1}{2} \sum_{i=1}^{2} S u p W_{\omega_{i}, m s} \exp \left(-\tau_{\delta_{m s}} c_{\omega_{i}, m s, B V}^{V R} V R_{\omega_{i}, m s}^{*}\right)
$$

where $c_{\omega_{i}, m s, B V}^{V R}, i=1,2$ are the scaling constants appropriate for the individual frequency $\operatorname{Sup} W_{\omega_{i}, m s, B V}(V R)$ statistics $i=1,2$.

Relevant values of $\tau_{\delta_{m}}$ and $\tau_{\delta_{m s}}$ that yield robust tests will be given in section S.1.2 in the supplement to this paper.

\section{LARGE SAMPLE RESULTS}

The supplement provides representations for the limiting distributions of the $W_{\omega_{i}, m}(U R)$ and $\operatorname{Sup} W_{\omega_{i}, m s}(V R), i=1,2$, statistics proposed in section 3, together with the corresponding joint frequency test statistics from section 3.5, under both the null and local alternative hypotheses. The form of the latter depend on whether the data are stationary or integrated at the frequency (or frequencies in the case of the joint frequency tests) of interest. We first define these local alternatives formally. The relevant local alternatives when testing for seasonal spectral means or for seasonal spectral mean shifts are now given in Definitions 4.1 and 4.2, respectively.

Definition 4.1. Let the filtered data $x_{i, t}, i=1,2$, be generated according to (3.4). Then: (a) if the data are $I_{\omega_{i}}(0), \delta_{\omega_{i}, m}:=\delta_{\omega_{i}, m, T}=T^{-1 / 2} \kappa_{\omega_{i}, m}$ and $\delta_{\omega_{i}, m s}=0$; (b) if the data are $I_{\omega_{i}}(1), \delta_{\omega_{i}, m}:=\delta_{\omega_{i}, m, T}=T^{1 / 2} \kappa_{\omega_{i}, m}$ and $\delta_{\omega_{i}, m s}=0$. In each case $\kappa_{\omega_{i}, m}$ is a finite constant.

REMARK 4.1. The scaling of the $\delta_{\omega_{i}, m}$ coefficients in (3.4) by powers of $T^{-1 / 2}$ in (a) and by $T^{1 / 2}$ in (b) provide the appropriate Pitman localisation rates when the data are $I_{\omega_{i}}(0)$ and $I_{\omega_{i}}(1)$, respectively, with the Pitman drift parameters in each case given by $\kappa_{\omega_{i}, m}$. Notice that we have set $\delta_{\omega_{i}, m s}=0$ in Definition 4.1 because the seasonal spectral mean tests outlined in this paper are constructed under the maintained assumption that no level break is present in the seasonal deterministic component.

Definition 4.2. Let the filtered data $x_{i, t}, i=1,2$, be generated according to (3.4). Then: (a) if the data are $I_{\omega_{i}}(0), \delta_{\omega_{i}, m s}:=\delta_{\omega_{i}, m s, T}=T^{-1 / 2} \kappa_{\omega_{i}, m s}$; (b) if the data are $I_{\omega_{i}}(1), \delta_{\omega_{i}, m s}:=\delta_{\omega_{i}, m s, T}=T^{1 / 2} \kappa_{\omega_{i}, m s}$. In each case $\kappa_{\omega_{i}, m s}$ is a finite constant.

REMARK 4.2. Notice that no restrictions are placed on the parameters $\delta_{\omega_{i}, m}$ in (3.4) by 
Definition 4.2 because the inclusion of $z_{i, t}$ in regression (3.6) renders the seasonal spectral mean shift tests exact invariant to these parameters.

Remark 4.3. Notice that the local alternatives given for $\delta_{\omega_{1}, m}$ and $\delta_{\omega_{2}, m}$ in Definition 4.1 reduce, respectively, to the null hypothesis $H_{0}^{\omega_{1}, m}$ when $\kappa_{\omega_{1}, m}=0$ and to $H_{0}^{\omega_{2}, m}$ when $\kappa_{\omega_{2}, m}=0$. Similarly, the local alternatives for $\delta_{\omega_{1}, m s}$ and $\delta_{\omega_{2}, m s}$ in Definition 4.2 reduce to $H_{0}^{\omega_{1}, m s}$ when $\kappa_{\omega_{1}, m s}=0$ and $H_{0}^{\omega_{2}, m s}$ when $\kappa_{\omega_{2}, m s}=0$, respectively.

Representations for the limiting distributions under the relevant local alternative for the annual frequency spectral mean statistics, $W_{\omega_{1}, m, V}(U R)$ and $W_{\omega_{1}, m, B V}(U R)$, are given in Theorems S.2 and S.3 for the case where the data are $I_{\omega_{1}}(0)$ and $I_{\omega_{1}}(1)$, respectively. Corresponding results for the Nyquist frequency statistics, $W_{\omega_{2}, m, V}(U R)$ and $W_{\omega_{2}, m, B V}(U R)$, are given in Theorems S.4 and S.5 for the case where the data are $I_{\omega_{2}}(0)$ and $I_{\omega_{2}}(1)$, respectively. The corresponding limiting distributions for the annual frequency mean shift statistics, $\operatorname{Sup}_{\omega_{1}, m s, V}(V R)$ and $\operatorname{Sup}_{\omega_{1}, m s, B V}(V R)$ are given in Theorems S.7 and S.8 for the case where the data are $I_{\omega_{1}}(0)$ and $I_{\omega_{1}}(1)$, respectively. Finally, corresponding results for the Nyquist frequency mean shift statistics, $S u p W_{\omega_{2}, m s, V}(V R)$ and $S u p W_{\omega_{2}, m s, B V}(V R)$ are given in Theorems S.9 and S.10 for the case where the data are $I_{\omega_{2}}(0)$ and $I_{\omega_{2}}(1)$, respectively.

The results in Theorems S.2-S.5 and S.7-S.10 show that for all of the statistics, where the data are stationary at the frequency under test, then so the limiting distributions of the statistics are unaffected by the choice of unit root statistic employed in the scale factor used in their construction. This is because, the unit root statistics converge in probability to zero in large samples, such that the multiplicative exponential functions used in the scale factors converges in probability to unity. Where the data are integrated at the frequency of interest the limiting distributions of the statistics now also involve the limiting distribution of the seasonal unit root statistic used in their construction. In section 4.1 we will discuss how appropriate values of the scaling factor constants can be chosen in order to yield tests that are robust to whether the data are stationary or integrated at the frequency under test.

The results in Theorems S.2-S.5 and S.7-S.10 also show that the limiting distributions of each of the annual frequency test statistics, either for spectral means or for spectral mean shifts, are independent of the limiting distributions of each of the corresponding Nyquist frequency test statistics. This is because the terms which feature in the limiting distributions of the statistics at the annual and Nyquist frequencies are formed from independent Brownian motions. An implication of this is that the limiting distributions of the joint frequency statistics in section 3.5 are simply the averages of the limiting distributions of their two constituent statistics.

\subsection{Asymptotic Null Distributions}

The limiting null distributions of the $W_{\omega_{i}, m}(U R)$ and $S u p W_{\omega_{i}, m s}(V R), i=1,2$, statistics under their respective null hypotheses, $H_{0}^{\omega_{i}, m}$ and $H_{0}^{\omega_{i}, m s}, i=1,2$, are obtained from the limiting distributions given in Theorems S.2-S.5 and Theorems S.7-S.10 by setting the relevant Pitman drift parameter from Definitions 4.1 and 4.2, respectively, to zero.

The limiting null distributions of all of the test statistics conducted at frequency $\omega_{i}$, $i=1,2$, are seen to be asymptotically free of nuisance parameters when the data are 
$I_{\omega_{i}}(0)$. For the $W_{\omega_{i}, m, V}(U R)$ and $\operatorname{Sup} W_{\omega_{i}, m s, V}(V R)$ test statistics, these distributions depend on $\bar{\alpha}_{i}$ and $c_{\omega_{i}, m, V}^{U R}$ and $c_{\omega_{i}, m s, V}^{V R}$, respectively, when the data are $I_{\omega_{i}}(1)$. Denoting the $\xi \%$ critical value from the limiting null distribution of any individual frequency $\omega_{i}$ statistic when the data are $I_{\omega_{i}}(0)$ as $c v_{0, \xi}$, a robust test can be performed by selecting a value of the scaling constant $c_{\omega_{i}, m, V}^{U R}$ or $c_{\omega_{i}, m s, V}^{V R}$ such that when the test statistic is compared to $c v_{0, \xi}$, the asymptotic size of the test procedure does not exceed $\xi \%$ when the data are $I_{\omega_{i}}(1)$ for a range of values of $\bar{\alpha}_{i}$. The asymptotic null distributions of the $W_{\omega_{i}, m, B V}(U R)$ and $S u p W_{\omega_{i}, m s, B V}(V R)$ statistics additionally depend on the bandwidth fraction, $b$, and kernel, $k(\cdot)$, associated with the long run variance estimator. Thus, for given $b$ and $k(x)$, if we denote the $\xi \%$ critical value from the limiting null distribution of an individual frequency $\omega_{i}$ statistic when the data are $I_{\omega_{i}}(0)$ as $c v_{0, b, k(\cdot), \xi}$, we can again choose a value of the scaling constant $c_{\omega_{i}, m, B V}^{U R}$ or $c_{\omega_{i}, m s, B V}^{V R}$ such that, when compared to $c v_{0, b, k(\cdot), \xi}$, the test has size that does not exceed $\xi \%$ when the data are $I_{\omega_{i}}(1)$ for a range of values of $\bar{\alpha}_{i}$ when using a bandwidth $b$ and kernel $k(\cdot)$.

In order to determine the critical values and scaling constant for the tests, the asymptotic null distributions of the test statistics outlined in Theorems S.2-S.5 and Theorems S.7-S.10 were simulated using Monte Carlo methods. The Brownian motion processes appearing in the asymptotic distributions were approximated using i.i.d. standard normal random variates discretised over 1000 steps. All simulations were carried out using 10,000 replications and with the trimming parameter set to $\lambda^{*}=0.1$. The range of values of $\bar{\alpha}_{i}$ considered when determining the scaling constants was set to $\bar{\alpha}_{i} \in\left[0, \bar{\alpha}_{i, \max }\right]$, where $\bar{\alpha}_{i, \max }$ was chosen such that the (asymptotic) size of all of the tests considered was maximised at $5 \%$ for some $\bar{\alpha}_{i} \in\left[0, \bar{\alpha}_{i, \max }\right]$. The asymptotic $5 \%$ critical values and appropriate scaling constants for the tests when using the Daniell kernel are reported in Tables S.1-S.4 in Section S.1.2 in the supplement to this paper. ${ }^{6}$

For the joint frequency tests discussed in section 3.5, we require values of the joint scaling constants $\tau_{\delta_{m}}$ and $\tau_{\delta_{m s}}$, such that when using the scaling constants $c_{\omega_{i}, m, B V}^{V R}$ or $c_{\omega_{i}, m s, B V}^{V R} i=1,2$, appropriate for the individual statistics, the asymptotic sizes of the joint $J S_{\delta_{m}}(V R)$ and $J S_{\delta_{m s}}(V R)$ tests do not exceed $\xi \%$ for for a large range of $\bar{\alpha}_{i}, i=1,2$ when the data are, potentially, $I_{\omega_{i}}(1) i=1,2$. Asymptotic critical values and appropriate values of $\tau_{\delta_{m}}$ and $\tau_{\delta_{m s}}$ for nominal $5 \%$ tests when constructing the $J S_{\delta_{m}}(V R)$ and $J S_{\delta_{m s}}(V R)$ tests are reported in Tables S.5 and S.6 respectively in section S.1.2 in the supplement to this paper. Notice that these are functions of the bandwidth fractions $b_{W_{\omega_{i}, m}}$ and $b_{S u p W_{\omega_{i}, m s}}, i=1,2$, used to construct the individual $W_{\omega_{i}, m}(V R)$ and $\operatorname{Sup} W_{\omega_{i}, m s}(V R)$ statistics.

\subsection{Asymptotic Local Power}

We next explore how the asymptotic local power of the test statistics that use the $\sigma_{B V, \omega_{i}}^{2}$ variance estimate can be used to determine the optimal bandwidth to use for a given value of $\bar{\alpha}_{i}$. The asymptotic distributions of the test statistics were simulated using the same Monte Carlo methods used for simulating the limiting null distributions and power for various local alternatives was computed. In all simulations we set $\lambda^{*}=0.1$

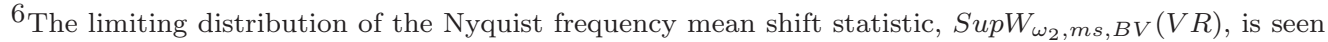
to be identical to that of the zero frequency mean shift test based on Model (1) of Sayginsoy and Vogelsang (2011) under both the null and local alternative hypotheses. As such, critical values and the appropriate scaling constant used to construct the $S u p W_{\omega_{2}, m s, B V}(V R)$ test statistic, again for the case of the Daniell kernel, can be taken directly from Table 1.1.1 of Sayginsoy and Vogelsang (2008).
} 
and $\lambda_{0}=0.5$. We restricted our attention to this one parameter setting because of the intensive computational requirements when simulating the limiting distributions under local alternatives. ${ }^{7}$ Using the $5 \%$ asymptotic critical values and scaling factors reported in Section S.1.2, asymptotic local power was computed for the case where the data were $I_{\omega_{i}}(0)$ and $I_{\omega_{i}}(1)$ for a range of values of $\bar{\alpha}_{i}$.

For individual frequency $\omega_{i}, i=1,2$, tests using the $\sigma_{B V, \omega_{i}}^{2}$ variance estimator, local power depends on the bandwidth, $b$ and kernel, $k(\cdot)$, used to construct this variance estimator when the series is $I_{\omega_{i}}(0)$ or $I_{\omega_{i}}(1)$, and additionally depends on $\bar{\alpha}_{i}$ when the series is $I_{\omega_{i}}(1)$. It is also the case that no single bandwidth maximises asymptotic local power uniformly for all local alternatives for any individual test; in particular, the asymptotic local power curves for tests constructed using different values of the bandwidth fraction $b$ for a given kernel $k(\cdot)$ cross one another. Consequently, we proceed in the same manner as Sayginsoy and Vogelsang (2011) to determine the optimal bandwidth fraction, $b$ to use both when the series are $I_{\omega_{i}}(0)$ or $I_{\omega_{i}}(1)$ by evaluating integrated asymptotic power.

To that end, let $\zeta_{\delta}\left(\bar{\alpha}_{i}, b, k(\cdot), \delta_{\omega_{i}, T}, \xi\right)$ denote the limiting distribution of an individual frequency $\omega_{i}$ statistic constructed using the appropriate scaling constant for a $\xi$ level test under the local alternative $\delta_{\omega_{i}, T}$ and where the kernel function and bandwidth fraction in (3.8) are given by $k(\cdot)$ and $b$, respectively. Asymptotic local power is given by $P\left[\zeta_{\delta}\left(\bar{\alpha}_{i}, b, k(\cdot), \delta_{\omega_{i}, T}, \xi\right)>c v_{0, b, k(\cdot), \xi}\right]$ where $c v_{0, b, k(\cdot), \xi}$ is the $\xi \%$ asymptotic critical value of the test statistic when the data are $I_{\omega_{i}}(0)$. Consequently, the integrated asymptotic local power of the test is given by

$$
\int_{0}^{\infty}\left[P\left[\zeta_{\delta}\left(\bar{\alpha}_{i}, b, k(\cdot), \delta_{\omega_{i}, T}, \xi\right)>c v_{0, b, k(\cdot), \xi}\right]\right] d\left(\delta_{\omega_{i}, T}\right)
$$

which can be computed using numerical integration methods. For a given $\bar{\alpha}_{i}$, the maximum value of $\delta_{\omega_{i}, T}$ is chosen such that the asymptotic local power of any given test is equal to at least 0.99 for at least one bandwidth fraction $b$. Using this method we find values of $b$, when using the Daniell kernel, such that integrated power is maximised for a given $\bar{\alpha}_{i}$ over a grid of values of $\bar{\alpha}_{i}$ selected such that the optimal bandwidth fraction for the largest value of $\bar{\alpha}_{i}$ is equal to the lowest bandwidth fraction considered of $b=0.02$. The optimal bandwidth fraction for each test is larger for smaller values of $\bar{\alpha}_{i}$, declining to 0.02 as $\bar{\alpha}_{i}$ increases. A similar analysis when the process is $I_{\omega_{i}}(0)$ confirmed that $b=0.02$ is also optimal in this instance. Optimal bandwidth fractions for all test statistics using the $\sigma_{B V, \omega_{i}}^{2}$ variance estimate are given in section S.1.1 in the supplement. ${ }^{8}$

\section{ADDITIONAL PRACTICAL IMPLEMENTATION ISSUES}

\subsection{Bandwidth Selection}

In the previous section we derived optimal bandwidth functions for tests based on the $\sigma_{B V, \omega_{i}}^{2}$ variance estimator using the Daniell kernel. These involve the non-centrality parameters $\bar{\alpha}_{i} i=1,2$, which are unknown in practice and cannot be consistently estimated. We can, however, use a feasible data-dependent bandwidth rule to select the bandwidth.

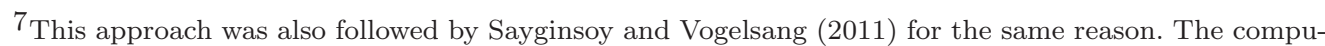
tational requirements for the simulations performed here are more onerous than those in Sayginsoy and Vogelsang (2011) because the variance estimates used in our tests are matrices rather than scalars.

${ }^{8}$ The optimal bandwidth fraction, $b$, when constructing the Nyquist frequency mean shift test $S u p W_{\omega_{2}, m s, B V}(V R)$ using the Daniell kernel, is again identical to the optimal bandwidth rule for Model (1) of Sayginsoy and Vogelsang (2011) reported in Figure 1.1 of Sayginsoy and Vogelsang (2008). 
Concentrating initially on tests at the Nyquist frequency, because $\bar{\alpha}_{2}=T\left(1-\alpha_{2}\right)$ we can propose a similar method to that used by Bunzel and Vogelsang (2005) and Sayginsoy and Vogelsang (2011) to obtain an estimate of $\bar{\alpha}_{2}$. Specifically, estimate by OLS

$$
\breve{u}_{2, t}=-\hat{\alpha}_{2} \breve{u}_{2, t-1}+\hat{e}_{t}, t=4, \ldots, T,
$$

where $\breve{u}_{2, t}$ are the residuals from the OLS regression in (3.5) when testing for a Nyquist frequency mean, or in the case of the tests for a level break at the Nyquist frequency the residuals from regression (3.6) evaluated at the break date $T_{b, N} \in \Lambda^{*}$, where $T_{b, N}$ is chosen such that it minimises the sum of squared residuals $\sum_{t=f_{2}}^{T} \breve{u}_{2, t}^{2}$. We then compute $\hat{\bar{\alpha}}_{2}:=T\left(1-\hat{\alpha}_{2}\right)$. The test can then be performed using the optimal bandwidth corresponding to the estimated value of $\bar{\alpha}_{2}$.

The same approach can be used for selecting a bandwidth in connection with the annual frequency tests. Specifically, we first estimate the OLS regression,

$$
\breve{u}_{1, t}=-\hat{\alpha}_{1}^{2} \breve{u}_{1, t-2}+\hat{v}_{t}, t=4, \ldots, T,
$$

where $\breve{u}_{1, t}$ are the residuals from the OLS regression in (3.5) when testing for an annual frequency mean, or for the annual frequency level break test the residuals from regression (3.6) evaluated at the break date $T_{b, A} \in \Lambda^{*}$, where $T_{b, A}$ is chosen such that it minimises the sum of squared residuals $\sum_{t=f_{1}}^{T} \breve{u}_{1, t}^{2}$. If $\hat{\alpha}_{1}^{2} \leq 0$ we perform the test using the optimal bandwidth for when the data is stationary at the annual frequency, otherwise we compute $\hat{\bar{\alpha}}_{1}:=T\left(1-\hat{\alpha}_{1}\right)$. The test can then be performed using the optimal bandwidth for the estimated value of $\bar{\alpha}_{1} .{ }^{9}$

\subsection{Allowing for Zero Frequency Linear and Broken Linear Trends}

Although the specification of $Z_{t}$ in (2.4) does not include a zero frequency linear trend, the filters applied to the data in (3.2) and (3.3) would reduce a zero frequency linear trend, should one be present in $Z_{t}$, to a zero frequency intercept in the filtered data, $x_{i, t}, i=1,2$. Consequently, because (3.5) and (3.6) both include a zero frequency intercept, a linear trend in $Z_{t}$ is already allowed for in our proposed seasonal frequency test procedures.

If one was concerned about the possibility of a single break in the zero frequency trend function, two choices could be made. First, the zero frequency trend break test of Sayginsoy and Vogelsang (2011) could be performed on $x_{0, t}$ of (3.1) as a pre-test, and the data de-trended accordingly if a broken trend was signalled. Alternatively, the tests outlined in this paper could be constructed using the filtered data $\tilde{x}_{i, t}:=(1-L) x_{i, t}$, $i=1,2$. This transforms the zero frequency trend break to a single outlier that would have no impact on the large sample properties of the seasonal frequency tests. Notice that one does not need to know or estimate the location of any zero frequency trend break in this latter approach, which is also asymptotically valid for a more general segmented zero frequency trend function, provided the number of breaks is fixed.

\subsection{Practical Recommendations}

In the supplement to this paper we report results from a Monte Carlo study examining the finite sample size and power properties of the tests proposed in this paper. These results

\footnotetext{
${ }^{9}$ Notice that we do not include $\breve{u}_{1, t-1}$ as a regressor in (5.2), consistent with the definition of nearintegration at the annual frequency which sets $\beta_{1}=0$ in $(2.3)$.
} 
suggest that when testing for seasonal spectral means the $W_{\omega_{i}, m, B V}(V R), i=1,2$, tests display the best size and power performance across the tests for spectral means at the annual and Nyquist frequencies, respectively. Likewise, when testing for seasonal spectral mean shifts the results suggest that the $\operatorname{Sup} W_{\omega_{i}, m s, B V}(V R), i=1,2$, tests display the best size and power performance when testing for spectral mean shifts at the annual and Nyquist frequencies, respectively. We therefore recommend the use of the $W_{\omega_{i}, m, B V}(V R)$, $i=1,2$, and the associated joint seasonal frequency test for deterministic seasonality, $J S_{\delta_{m}}(V R)$ when testing for seasonal means, and when testing for seasonal mean shifts we recommend the use of the $\operatorname{Sup}_{\omega_{i}, m s, B V}(V R) i=1,2$, tests and the associated joint seasonal frequency test, $J S_{\delta_{m s}}(V R)$. In each case these tests should be constructed using the Daniell kernel and using the bandwidth selection rules outlined in Section 5.1.

\section{AN EMPIRICAL APPLICATION TO U.K. GDP}

We now provide an empirical example using quarterly unadjusted real GDP data from 1997Q1-2015Q4 taken from the ONS website. Our aim is to investigate whether or not the deterministic seasonal pattern in these data is constant across the sample period. Visual inspection of the data in Figure 1 is suggestive of the presence of a segmented zero frequency linear trend in the data, characterised by a negative growth rate in real GDP during the financial crisis of 2007-2009 in a series otherwise exhibiting positive long run growth. A segmented zero frequency trend of this form would lead to zero frequency level shifts in the filtered data used to construct the seasonal frequency tests outlined in this paper. We therefore first de-trend the data allowing for two trend breaks, with the break dates chosen so as to minimise the residual sum of squares from the de-trending regression. The fitted zero frequency segmented trend is also graphed in Figure 1.

Table 1: Test Results for Empirical Application

\begin{tabular}{rccc}
\hline Test & Test Statistic & Critical Value & Break Date Identified \\
\hline Sup $W_{\omega_{2}, m s, B V}(V R)$ & 16.63 & 14.45 & $2006 \mathrm{Q} 4$ \\
Sup $W_{\omega_{1}, m s, B V}(V R)$ & 30.76 & 28.77 & $2009 \mathrm{Q} 2$ \\
$J S_{\delta_{m s}}(V R)$ & 27.93 & 21.61 & NA \\
\hline
\end{tabular}

In Table 1 we report the results of applying the tests for the presence of a seasonal mean shift at the annual and Nyquist frequencies, $S u p W_{\omega_{1}, m s, B V}(V R)$ and $S u p W_{\omega_{2}, m s, B V}(V R)$, respectively, together with the joint frequency seasonal mean shift test, $J S_{\delta_{m s}}(V R)$, to the de-trended data. All tests are run at the nominal asymptotic $5 \%$ level in each case using the Daniell kernel and the bandwidth selection rules outlined in Section 5.1. All of the tests reject the null hypothesis of no level break. The timing of the break, identified by the estimated break date that led to the largest Wald statistic in the construction of the $S u p W_{\omega_{i}, m s, B V}(V R)$ test statistic, is estimated to be 2006Q4 for the Nyquist frequency deterministic component and 2009Q2 for the annual frequency deterministic component. Although these estimated break dates are at different points in the sample they do, however, straddle the onset of the financial crisis in 2007-08. ${ }^{10}$

${ }^{10}$ We also considered the approach outlined in Section 5.2 wherein the test statistics are applied to the filtered data $\tilde{x}_{i, t}, i=1,2$. This yielded similar results to those reported above for the de-trended data, although the $\operatorname{Sup} W_{\omega_{1}, m s, B V}(V R)$ test failed to reject the null of no shift in mean at the annual frequency. The break dates identified using this approach only differed from those found using the de- 


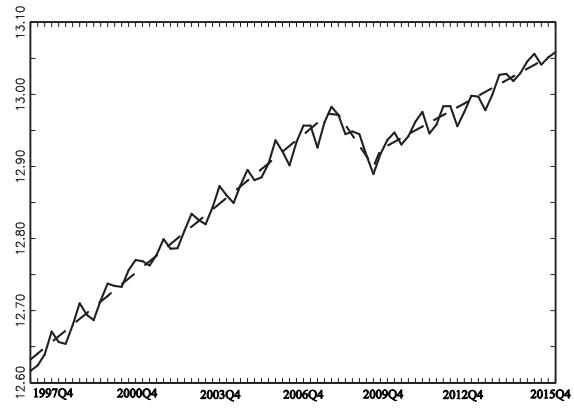

Figure 1. Fitted Segmented Trend Series: — Fitted Segmented Trend: . . . . .

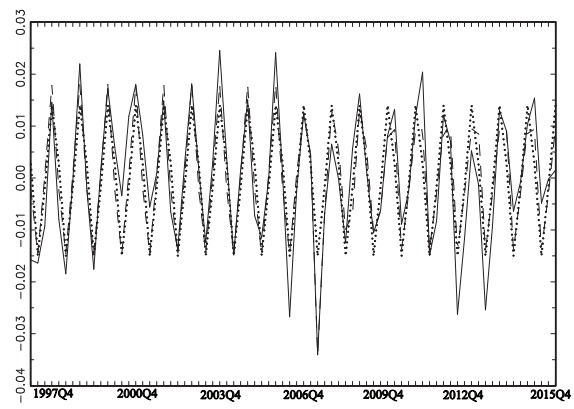

Figure 2. Fitted Deterministic Component

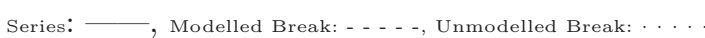

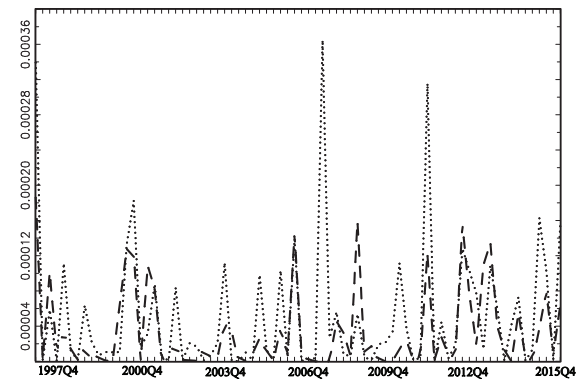

Figure 3. Squared Estimation Errors in Detrended Data Series: — Modelled Break: - . . . - Unmodelled Break: . . . .

We next estimate the deterministic component in the de-trended GDP series both for the case where we allow for the broken deterministic seasonal components identified by our test procedures, and where it is assumed that there are no seasonal mean shifts. In the former case we include the $f_{i}$ impulse dummies after the break identified at frequency $\omega_{i}$ to eliminate the effect of any outliers in the filtered data caused by the break; cf. (3.4). Figure 2 plots the detrended GDP series against the fitted seasonal deterministic components for both cases. This graph suggests that both fitted series appear to track the detrended data reasonably well for the majority of the sample. However, the impact of failing to model the identified seasonal level breaks can be more clearly seen in Figure 3 which graphs the squared deviations of each fitted deterministic component from the detrended series. The model which does not allow for a break in deterministic seasonality at the time of the financial crisis is prone to making very large errors in the post crisis period. For the period 2010Q1-2015Q4 (chosen such that the sample contains no indicator dummies to ensure a fair comparison) the mean square error [MSE] for the model where the seasonal level breaks are accounted for is 0.0000401 , which is around $40 \%$ lower than the MSE of 0.0000658 for the model which does not allow for seasonal level breaks. It therefore seems likely that not allowing for the seasonal mean shifts identified by our proposed tests would have had an adverse affect on the quality of the seasonally adjusted data and any forecasts made in the post-crisis period.

trended data by one quarter at each frequency, with a mean shift at the Nyquist frequency detected in 2007Q1 and a mean shift at the annual frequency most likely to have occurred in 2009Q3. 


\section{CONCLUSIONS}

We have proposed tests for the presence of deterministic seasonality and breaks in deterministic seasonality in seasonally observed time series which are designed to be asymptotically robust to the order of integration of the data at both the zero and seasonal frequencies. Simulation results in the supplementary paper show that our proposed tests have good size control in finite samples. Recommendations have been provided for how to perform these tests in practice. In an empirical example we have shown that quarterly seasonally unadjusted U.K. GDP appears to have been subject to a significant shift in its deterministic seasonal pattern around the time of the financial crisis.

\section{REFERENCES}

Bunzel, H. and Vogelsang, T.J. (2005). Powerful trend function tests that are robust to strong serial correlation, with an application to the Prebisch-Singer hypothesis. Journal of Business and Economic Statistics 23, 381-394.

Franses, P.H. and Vogelsang, T.J. (1998). On seasonal cycles, unit roots, and mean shifts. The Review of Economics and Statistics 80, 231-240.

Harvey, D., Leybourne, S., and Taylor, R. (2006). On robust trend function hypothesis testing. Studies in Non-Linear Dynamics and Econometrics 10:1, Article 1.

Harvey, D.I., Leybourne, S.J., and Taylor, A.M.R. (2007). A simple, robust and powerful test of the trend hypothesis. Journal of Econometrics 141, 1302-1330.

Hylleberg, S., Engle, R.F., Granger, C.W.J., and Yoo, B.S. (1990). Seasonal integration and cointegration. Journal of Econometrics 44, 215-238.

Miron, J. (1996). The Economics of Seasonal Cycles, Cambridge, MA: M.I.T. Press.

Park, J.Y. (1990). Testing for unit roots and cointegration by variable addition. In: Fomby, T., Rhodes, F. (Eds.), Advances in Econometrics: Cointegration, Spurious Regression and Unit Roots. Jai Press, Greenwich.

Park, J.Y. and Choi, B. (1988). A new approach to testing for a unit root. CAE Working Paper 88-23. Cornell University.

Perron, P. (1989) The great crash, the oil price shock, and the unit root hypothesis. Econometrica 57, 13611401.

Perron, P. and Yabu, T. (2009). Estimating deterministic trends with an integrated or stationary noise component. Journal of Econometrics 151, 56-69.

Sayginsoy, O. and Vogelsang, T.J. (2011). Testing for a shift in trend at an unknown date: A fixed-b analysis of heteroskedasticity autocorrelation robust OLS-based tests. Econometric Theory 27, 992-1025.

Sayginsoy, O. and Vogelsang, T.J. (2008). Testing for a Shift in Trend at an Unknown Date: A Fixed-b Analysis of Heteroskedasticity Autocorrelation Robust OLS Based Tests. Working paper, Department of Economics, Michigan State University.

Taylor A.M.R. (2005). Variance ratio tests of the seasonal unit root hypothesis. Journal of Econometrics 124, 33-54.

Vogelsang, T.J. (1998). Trend function hypothesis testing in the presence of serial correlation. Econometrica 66, 123-148. 


\title{
Robust Tests for Deterministic Seasonality and Seasonal Mean Shifts
}

\author{
S. Astill And A.M.R. TAYlor \\ Essex Business School, University of Essex, Wivenhoe Park, Colchester, Essex, CO4 3SQ, UK \\ E-mail: sastill@essex.ac.uk, rtaylor@essex.ac.uk
}

January 2018

\begin{abstract}
Summary The outline of this supplementary paper is as follows. Section S.1 provides numerical information required for practical implementation of the tests outlined in the main paper including asymptotic critical values and scaling constants and optimal bandwidth fractions. Section S.2 outlines the limiting distributions of the test statistics outlined in the paper. Section S.3 reports results of a Monte Carlo simulation exercise examining the asymptotic local power functions of the proposed tests. Results from a Monte Carlo simulation exercise examining the finite sample size and power properties of the tests are reported in Section S.4. Finally, Sections S.5 and S.6 provide proofs for the main theoretical results stated in the paper.
\end{abstract}

Keywords: Seasonality, Seasonal Unit Roots, Robust Tests approximation

\section{S.1. NUMERICAL INFORMATION FOR PRACTICAL IMPLEMENTATION}

S.1.1. Optimal Bandwidth Fractions for $W_{\omega_{i}, m, B V}(U R)$ and $S \operatorname{Sup}_{\omega_{i}, m s, B V}(V R)$, $i=1,2$.

Let $I(x<a)$ denote the indicator function that takes the value 1 if $x<a$ and 0 otherwise. We then have the following rules:

$$
\begin{aligned}
b_{\mathrm{opt}}\left(W_{\omega_{2}, m, B V}(V R)\right) & =0.02+\left(0.04 \times I\left(\bar{\alpha}_{2}<280\right)\right)+\left(0.02 \times I\left(\bar{\alpha}_{2}<150\right)\right)+\left(0.02 \times I\left(\bar{\alpha}_{2}<90\right)\right) \\
& +\left(0.04 \times I\left(\bar{\alpha}_{2}<85\right)\right)+\left(0.02 \times I\left(\bar{\alpha}_{2}<55\right)\right)+\left(0.04 \times I\left(\bar{\alpha}_{2}<50\right)\right) \\
& +\left(0.02 \times I\left(\bar{\alpha}_{2}<23\right)\right)+\left(0.02 \times I\left(\bar{\alpha}_{2}<15\right)\right)+\left(0.02 \times I\left(\bar{\alpha}_{2}<11\right)\right) \\
& +\left(0.04 \times I\left(\bar{\alpha}_{2}<8\right)\right) \\
b_{\text {opt }}\left(W_{\omega_{2}, m, B V}(H)\right)= & 0.06+\left(0.02 \times I\left(\bar{\alpha}_{2}<525\right)\right)+\left(0.06 \times I\left(\bar{\alpha}_{2}<235\right)\right)+\left(0.06 \times I\left(\bar{\alpha}_{2}<120\right)\right) \\
+ & \left(0.02 \times I\left(\bar{\alpha}_{2}<70\right)\right)+\left(0.04 \times I\left(\bar{\alpha}_{2}<39\right)\right)+\left(0.02 \times I\left(\bar{\alpha}_{2}<18\right)\right) \\
+ & \left(0.14 \times I\left(\bar{\alpha}_{2}<13\right)\right)+\left(0.12 \times I\left(\bar{\alpha}_{2}<6\right)\right)+\left(0.08 \times I\left(\bar{\alpha}_{2}<3\right)\right) \\
+ & \left(0.22 \times I\left(\bar{\alpha}_{2}<2\right)\right), \\
b_{\mathrm{opt}}\left(W_{\omega_{2}, m, B V}(J)\right)= & 0.02+\left(0.02 \times I\left(\bar{\alpha}_{2}<110\right)\right)+\left(0.02 \times I\left(\bar{\alpha}_{2}<38\right)\right)+\left(0.04 \times I\left(\bar{\alpha}_{2}<25\right)\right) \\
+ & \left(0.16 \times I\left(\bar{\alpha}_{2}<18\right)\right)+\left(0.06 \times I\left(\bar{\alpha}_{2}<13\right)\right)+\left(0.2 \times I\left(\bar{\alpha}_{2}<10\right)\right) \\
+ & \left(0.06 \times I\left(\bar{\alpha}_{2}<6\right)\right)+\left(0.14 \times I\left(\bar{\alpha}_{2}<5\right)\right) .
\end{aligned}
$$




$$
\begin{aligned}
& b_{\text {opt }}\left(W_{\omega_{1}, m, B V}(V R)\right)=0.02+\left(0.02 \times I\left(\bar{\alpha}_{1}<42\right)\right)+\left(0.06 \times I\left(\bar{\alpha}_{1}<36\right)\right)+\left(0.02 \times I\left(\bar{\alpha}_{1}<27\right)\right) \\
& +\left(0.06 \times I\left(\bar{\alpha}_{1}<19\right)\right)+\left(0.06 \times I\left(\bar{\alpha}_{1}<2\right)\right), \\
& b_{\text {opt }}\left(W_{\omega_{1}, m, B V}(H)\right)=0.02+\left(0.02 \times I\left(\bar{\alpha}_{1}<20\right)\right)+\left(0.06 \times I\left(\bar{\alpha}_{1}<14\right)\right)+\left(0.08 \times I\left(\bar{\alpha}_{1}<13\right)\right) \\
& +\left(0.02 \times I\left(\bar{\alpha}_{1}<11\right)\right)+\left(0.06 \times I\left(\bar{\alpha}_{1}<8\right)\right)+\left(0.06 \times I\left(\bar{\alpha}_{1}<5\right)\right) \\
& +\left(0.06 \times I\left(\bar{\alpha}_{1}<3\right)\right)+\left(0.48 \times I\left(\bar{\alpha}_{1}<1\right)\right) \\
& b_{\text {opt }}\left(W_{\omega_{1}, m, B V}(J)\right)=0.02+\left(0.02 \times I\left(\bar{\alpha}_{1}<66\right)\right)+\left(0.02 \times I\left(\bar{\alpha}_{1}<32\right)\right)+\left(0.02 \times I\left(\bar{\alpha}_{1}<17\right)\right) \\
& +\left(0.02 \times I\left(\bar{\alpha}_{1}<14\right)\right)+\left(0.02 \times I\left(\bar{\alpha}_{1}<12\right)\right)+\left(0.06 \times I\left(\bar{\alpha}_{1}<9\right)\right) \\
& +\left(0.02 \times I\left(\bar{\alpha}_{1}<7\right)\right)+\left(0.04 \times I\left(\bar{\alpha}_{1}<4\right)\right)+\left(0.08 \times I\left(\bar{\alpha}_{1}<3\right)\right) . \\
& b_{\mathrm{opt}}\left(S u p W_{\omega_{2}, m s, B V}(V R)\right)=0.02+\left(0.02 \times I\left(\bar{\alpha}_{2}<73\right)\right)+\left(0.02 \times I\left(\bar{\alpha}_{2}<56\right)\right)+\left(0.02 \times I\left(\bar{\alpha}_{2}<16\right)\right) \\
& +\left(0.02 \times I\left(\bar{\alpha}_{2}<2\right)\right) \\
& b_{\mathrm{opt}}\left(S u p W_{\omega_{1}, m s, B V}(V R)\right)=0.02+\left(0.02 \times I\left(\bar{\alpha}_{1}<19\right)\right)+\left(0.02 \times I\left(\bar{\alpha}_{1}<10\right)\right)+\left(0.02 \times I\left(\bar{\alpha}_{1}<1\right)\right)
\end{aligned}
$$

Table S.1: Asymptotic Critical Values and Scaling Constants for $5 \%$ level $W_{\omega_{i}, m, V}(U R)$ and $S u p W_{\omega_{i}, m s, V}(U R), i=1,2$, Tests.

\begin{tabular}{ccccc} 
& $5 \%$ Critical Value & $c_{\omega_{i}, m, V}^{V R}$ & $c_{\omega_{i}, m, V}^{H}$ & $c_{\omega_{i}, m, V}^{J}$ \\
\hline$W_{\omega_{2}, m, V}(U R)$ & 44.010 & 153.85 & 4.758 & 0.748 \\
$W_{\omega_{1}, m, V}(U R)$ & 210.714 & 96.159 & 11.215 & 1.044 \\
\hline & $5 \%$ Critical Value & $c_{\omega_{i}, m s, V}^{V R}$ & \\
\hline SupW & & \\
Sup $W_{\omega_{1}, m s, V}(U R)$ & 215.088 & 402.000 & & \\
\hline
\end{tabular}


Robust Tests for Deterministic Seasonality and Seasonal Mean Shifts

Table S.2: Asymptotic Critical Values and Scaling Constants for $5 \%$ level Annual Frequency $W_{\omega_{1}, m, B V}(U R)$ Tests.

\begin{tabular}{rrrrr}
\hline & & & & \\
& $c v(b)$ & $c_{\omega_{1}, m, B V}^{V R}(b)$ & $c_{\omega_{1}, m, B V}^{H}(b)$ & $c_{\omega_{1}, m, B V}^{J}(b)$ \\
\hline$a_{0}$ & 11.5 & 335.5 & 37.6 & 5.9 \\
$a_{1}$ & 67.0 & $-5,803.3$ & -644.8 & -177.0 \\
$a_{2}$ & 424.1 & $65,544.4$ & $8,097.3$ & $2,525.8$ \\
$a_{3}$ & $-2,254.0$ & $-457,682.8$ & $-62,636.4$ & $-19,555.8$ \\
$a_{4}$ & $17,083.0$ & $2,022,737.4$ & $298,124.5$ & $90,260.9$ \\
$a_{5}$ & $-29,552.6$ & $-5,791,836.4$ & $-899,309.7$ & $-262,070.6$ \\
$a_{6}$ & $35,716.7$ & $10,866,441.0$ & $1,750,990.3$ & $490,996.3$ \\
$a_{7}$ & $-13,658.5$ & $-13,242,716.0$ & $-2,191,372.4$ & $-592,765.3$ \\
$a_{8}$ & - & $10,083,384.0$ & $1,700,522.8$ & $445,367.2$ \\
$a_{9}$ & - & $-4,353,162.4$ & $-743,966.9$ & $-189,421.0$ \\
$a_{10}$ & - & $812,797.0$ & $140,162.2$ & $34,834.3$ \\
\hline
\end{tabular}

Note: For the $W_{\omega_{1}, m, B V}(U R)$ tests, in a similar manner to Bunzel and Vogelsang (2005), we report asymptotic $5 \%$ critical values $(c v(b))$ and appropriate scaling factors $\left(c_{\omega_{1}, m, B V}^{U R}(b)\right)$ as a function of the bandwidth fraction, $b$, estimated as a polynomial in $b$ given by $\sum_{i=0}^{i_{\max }} a_{i} b^{i}$, where $i_{\max }$ was chosen in each case such that the polynomial explained $99 \%$ of the variation of $c v(b)$ or $c_{\omega_{1}, m, B V}^{U R}(b)$.

Table S.3: Asymptotic Critical Values and Scaling Constants for $5 \%$ level Nyquist Frequency $W_{\omega_{2}, m, B V}(U R)$ Tests.

\begin{tabular}{rrrrr} 
& $c v(b)$ & $c_{\omega_{2}, m, B V}^{V R}(b)$ & $c_{\omega_{2}, m, B V}^{H}(b)$ & $c_{\omega_{2}, m, B V}^{J}(b)$ \\
\hline$a_{0}$ & 4.1 & 458.4 & 14.1 & 5.9 \\
$a_{1}$ & 7.4 & $7,881.4$ & -157.7 & -168.0 \\
$a_{2}$ & 59.9 & $90,784.1$ & $1,543.8$ & $2,412.8$ \\
$a_{3}$ & 10.0 & $-632,483.3$ & $-10,085.0$ & $-18,768.0$ \\
$a_{4}$ & 100.8 & $2,755,664.4$ & $42,654.8$ & $87,031.0$ \\
$a_{5}$ & -100.2 & $-7,731,519.4$ & $-118,429.6$ & $-254,290.4$ \\
$a_{6}$ & - & $14,180,044.2$ & $217,166.2$ & $479,867.0$ \\
$a_{7}$ & - & $-16,888,666.6$ & $-259,651.8$ & $-583,475.2$ \\
$a_{8}$ & - & $12,578,503.6$ & $194,195.8$ & $441,203.0$ \\
$a_{9}$ & - & $5,319,275.6$ & $-82,336.6$ & $-188,657.4$ \\
$a_{10}$ & - & $974,474.4$ & $15,088.4$ & $34,839.8$ \\
\hline
\end{tabular}

Note: For the $W_{\omega_{2}, m, B V}(U R)$ test we report asymptotic $5 \%$ critical values $(c v(b))$ and appropriate scaling factors $\left(c_{\omega_{2}, m, B V}^{U R}(b)\right)$ as a function of the bandwidth fraction, $b$, estimated as a polynomial in $b$ given by $\sum_{i=0}^{i_{\max }} a_{i} b^{i}$, where $i_{\max }$ was chosen in each case such that the polynomial explained $99 \%$ of the variation of $c v(b)$ or $c_{\omega_{2}, m, B V}^{U R}(b)$. 
Table S.4: Asymptotic Critical Values and Scaling Constants for $5 \%$ level $S u p W_{\omega_{i}, m s, B V}(U R), i=1,2$, Tests.

\begin{tabular}{rccccc}
\hline & \multicolumn{3}{c}{$\operatorname{Sup} W_{\omega_{1}, m s, B V}(U R)$} & \multicolumn{4}{c}{$\begin{array}{c}S u p W_{\omega_{2}, m s, B V}(U R) \\
b\end{array}$} & $5 \%$ Critical Value & $c_{\omega_{1}, m s, B V}^{V R}$ & $b$ & $5 \%$ Critical Value & $c_{\omega_{2}, m s, B V}^{V R}$ \\
\hline 0.02 & 28.773 & 571.910 & 0.02 & 10.552 & 864.438 \\
0.04 & 34.462 & 382.270 & 0.04 & 12.294 & 594.524 \\
0.06 & 41.961 & 293.802 & 0.06 & 14.449 & 446.607 \\
0.08 & 47.547 & 260.816 & 0.08 & 17.055 & 365.121 \\
- & - & - & 0.10 & 20.194 & 318.715 \\
\hline
\end{tabular}

Note: Because the optimal bandwidth fractions, $b$, for the $S u p W_{\omega_{i}, m s, B V}(V R)$, $i=1,2$, tests were found to only take a small number of values we simply report the asymptotic $5 \%$ critical value and appropriate scaling factors for these tests for the necessary range of values of $b$.

Table S.5: Values of $\tau_{\delta_{m}}$ for the $5 \%$ level $J S_{\delta_{m}}(V R)$ Test.

\begin{tabular}{cc|cccccc}
\hline \hline & & \multicolumn{6}{c}{$b_{W_{\omega_{1}, m}}$} \\
& \multicolumn{1}{c}{0.02} & 0.04 & 0.10 & 0.12 & 0.18 & 0.24 \\
\hline$b_{W_{\omega_{2}, m}}$ & 0.02 & 1.083 & 1.075 & 1.083 & 1.084 & 1.076 & 1.042 \\
& 0.06 & 1.078 & 1.071 & 1.062 & 1.068 & 1.075 & 1.021 \\
& 0.08 & 1.077 & 1.070 & 1.063 & 1.055 & 1.060 & 1.011 \\
& 0.10 & 1.066 & 1.072 & 1.063 & 1.064 & 1.046 & 1.011 \\
& 0.14 & 1.064 & 1.075 & 1.079 & 1.064 & 1.069 & 1.030 \\
0.16 & 1.065 & 1.084 & 1.085 & 1.071 & 1.065 & 1.045 \\
0.20 & 1.088 & 1.099 & 1.116 & 1.100 & 1.077 & 1.058 \\
0.22 & 1.086 & 1.107 & 1.118 & 1.116 & 1.087 & 1.075 \\
0.24 & 1.094 & 1.103 & 1.124 & 1.127 & 1.112 & 1.083 \\
0.26 & 1.094 & 1.121 & 1.129 & 1.127 & 1.138 & 1.075 \\
0.30 & 1.113 & 1.132 & 1.129 & 1.124 & 1.148 & 1.115 \\
\hline
\end{tabular}

Table S.6: Values of $\tau_{\delta_{m s}}$ for the $5 \%$ level $J S_{\delta_{m s}}(V R)$ Test.

\begin{tabular}{ll|cccc}
\hline \hline & & \multicolumn{4}{c}{$b_{S u p W_{\omega_{1}, m s}}$} \\
\hline$b_{\text {Sup }_{\omega_{2}, m s}}$ & 0.02 & 0.956 & 0.04 & 0.06 & 0.08 \\
& 0.04 & 0.933 & 0.924 & 0.949 & 0.946 \\
& 0.06 & 0.882 & 0.897 & 0.903 & 0.942 \\
& 0.08 & 0.856 & 0.868 & 0.885 & 0.903 \\
& 0.10 & 0.846 & 0.852 & 0.869 & 0.869 \\
\hline
\end{tabular}




\section{S.2. ASYMPTOTIC DISTRIBUTIONS}

In this section we detail the asymptotic distributions of the test statistics outlined in the main paper. In the following $\stackrel{(p)}{\rightarrow}$, denotes convergence in probability and $\stackrel{d}{\rightarrow}$ ' denotes convergence in distribution, in each case as the sample size diverges.

\section{S.2.1. Preliminaries}

Before proceeding we require the results of the following Lemma.

Lemma S.1. Let the filtered series $x_{i, t}$ be generated according to (3.4) and let Assumption 2.1 hold. Recalling the definitions of $u_{i, t}$ and $v_{i, t}, i=1,2$, given in Section 3.1, then as $T \rightarrow \infty$,

(a) If $\alpha_{2}=1-\bar{\alpha}_{2} / T$,

$$
T^{-1 / 2} \Omega_{2,1}^{-1 / 2} u_{2,\lfloor T r\rfloor} z_{2,\lfloor T r\rfloor} \stackrel{d}{\rightarrow} V_{\bar{\alpha}_{2}, 2}(r),
$$

where $V_{\bar{\alpha}_{2}, 2}(r)$ is a standard Ornstein-Uhlenbeck process, $V_{\bar{\alpha}_{2}, 2}(r):=\int_{0}^{r} e^{-\bar{\alpha}_{2}(r-s)} d W_{2}(s)$, with $W_{2}(r)$ a standard Brownian motion and

$$
\Omega_{2,1}:=\lim _{T \rightarrow \infty} T^{-1} E\left[\left(\sum_{i=f_{2}+1}^{T} z_{2, t} v_{2, t}\right)\left(\sum_{i=f_{2}+1}^{T} z_{2, t} v_{2, t}\right)^{\prime}\right] .
$$

(b) If $\left|\alpha_{2}\right|<1$,

$$
T^{-1 / 2} \Omega_{2,0}^{-1 / 2} \sum_{t=f_{2}+1}^{\lfloor T r\rfloor} u_{2, t} z_{2, t} \stackrel{d}{\rightarrow} W_{2}(r),
$$

where

$$
\Omega_{2,0}:=\lim _{T \rightarrow \infty} T^{-1} E\left[\left(\sum_{i=f_{2}+1}^{T} z_{2, t} u_{2, t}\right)\left(\sum_{i=f_{2}+1}^{T} z_{2, t} u_{2, t}\right)^{\prime}\right] .
$$

(c) If $\alpha_{1}=1-\bar{\alpha}_{1} / T$ and $\beta_{1}=0$,

$$
T^{-1 / 2} \Omega_{1,1}^{-1 / 2} u_{1,\lfloor T r\rfloor} z_{1,\lfloor T r\rfloor} \stackrel{d}{\rightarrow}(1 / 2)\left(V_{\bar{\alpha}_{1}, 1}(r), V_{\bar{\alpha}_{3}, 3}(r)\right)^{\prime},
$$

where $V_{\bar{\alpha}_{1}, i}(r), i=1,3$ are standard Ornstein-Uhlenbeck processes, $V_{\bar{\alpha}_{1}, i}(r):=\int_{0}^{r} e^{-\bar{\alpha}_{i}(r-s)} d W_{i}(s)$, $i=1,3$ with $W_{i}(r), i=1,3$ standard Brownian motions and

$$
\Omega_{1,1}:=\lim _{T \rightarrow \infty} T^{-1} E\left[\left(\sum_{i=f_{1}+1}^{T} z_{1, t} v_{1, t}\right)\left(\sum_{i=f_{1}+1}^{T} z_{1, t} v_{1, t}\right)^{\prime}\right] .
$$

(d) If $\alpha_{1}^{2}+\beta_{1}^{2}<1$,

$$
T^{-1 / 2} \Omega_{1,0}^{-1 / 2} \sum_{t=1}^{\lfloor T r\rfloor} u_{1, t} z_{1, t} \stackrel{d}{\rightarrow}\left(W_{1}(r), W_{3}(r)\right)^{\prime}
$$


where

$$
\Omega_{1,0}:=\lim _{T \rightarrow \infty} T^{-1} E\left[\left(\sum_{i=f_{1}+1}^{T} z_{1, t} u_{1, t}\right)\left(\sum_{i=f_{1}+1}^{T} z_{1, t} u_{1, t}\right)^{\prime}\right] .
$$

Proof. The proof of this Lemma follows directly from results provided in the proof of Theorem 4.1 of Taylor (2005).

Before proceeding we will replace the local alternatives given in Definitions 4.1 and 4.2 with Definitions S.1 and S.2, respectively, below. These additionally scale out nuisance parameter terms related to the variance parameters defined in Lemma S.1 above such that, for convenience, they do not appear in the representations which follow for the local limiting distributions.

Definition S.1. Let the filtered data $x_{i, t}, i=1,2$, be generated according to (3.4). Then: (a) if the data are $I_{\omega_{i}}(0), \delta_{\omega_{i}, m}:=\delta_{\omega_{i}, m, T}=\Omega_{i, 0}^{1 / 2} T^{-1 / 2} \kappa_{\omega_{i}, m}$ and $\delta_{\omega_{i}, m s}=0$; (b) if the data are $I_{\omega_{i}}(1), \delta_{\omega_{i}, m}:=\delta_{\omega_{i}, m, T}=\Omega_{i, 1}^{1 / 2} T^{1 / 2} \kappa_{\omega_{i}, m}$ and $\delta_{\omega_{i}, m s}=0$. In each case $\kappa_{\omega_{i}, m}$ is a finite constant.

Definition S.2. Let the filtered data $x_{i, t}, i=1,2$, be generated according to (3.4). Then: (a) if the data are $I_{\omega_{i}}(0), \delta_{\omega_{i}, m s}:=\delta_{\omega_{i}, m s, T}=\Omega_{i, 0}^{1 / 2} T^{-1 / 2} \kappa_{\omega_{i}, m s}$; (b) if the data are $I_{\omega_{i}}(1), \delta_{\omega_{i}, m s}:=\delta_{\omega_{i}, m s, T}=\Omega_{i, 1}^{1 / 2} T^{1 / 2} \kappa_{\omega_{i}, m s}$. In each case $\kappa_{\omega_{i}, m s}$ is a finite constant.

The following conditions also hold for the deterministic components in the series.

Definition S.3. (a) There exists $\left(k_{1} \times k_{1}\right)$ and $\left(k_{2} \times k_{2}\right)$ normalisation matrices $\tau_{1 T}$ and $\tau_{2 T}$, respectively, and $\left(k_{1} \times 1\right)$ and $\left(k_{2} \times 1\right)$ vectors of functions $F_{i, m}(r)$ and $F_{i, m s}(r)$ on $[0,1]$, respectively, such that

$$
\begin{aligned}
& {\left[\begin{array}{cc}
\tau_{1 T} & 0_{k_{1} \times k_{2}} \\
0_{k_{2} \times k_{1}} & \tau_{2 T}
\end{array}\right]\left[\begin{array}{c}
F_{i, m}(t) \\
F_{i, m s}(t, l)
\end{array}\right]=\left[\begin{array}{c}
F_{i, m}(t / T)+o(1) \\
F_{i, m s}(t / T, l)+o(1)
\end{array}\right] } \\
\Rightarrow & {\left[\begin{array}{c}
F_{i, m}(r) \\
F_{i, m s}(r, l)
\end{array}\right]:=F_{i}(r, l) }
\end{aligned}
$$

where $l$ denotes generically $\lambda$ or $\lambda_{0}, F_{i, m}(t)$ and $F_{i, m s}(t, l)$ are as defined in (3.6) and $0_{k_{1} \times k_{2}}\left(0_{k_{2} \times k_{1}}\right)$ represents an $\left(k_{1} \times k_{2}\right)\left(\left(k_{2} \times k_{1}\right)\right)$ matrix of zeros:

(b) $\int_{0}^{1} F_{i}(r, l) d r<\infty$ and $\operatorname{det}\left[\int_{0}^{1} F_{i}(r, l) F_{i}(r, l)^{\prime} d r\right]>0$

Remark S.1. Notice that because all of the deterministic variables considered in this paper are either seasonal intercepts or broken seasonal intercepts, the normalisation matrices $\tau_{1 T}$ and $\tau_{2 T}$ are always vectors of ones so that $F_{i, m}(t)=F_{i, m}(r)$ and $F_{i, m s}(t, l)=$ $F_{i, m s}(r, l)$.

Finally, in Definition S.4 we define functions featuring in the asymptotic distributions of the $W_{\omega_{i}, m}(U R)$ and $S u p W_{\omega_{i}, m s}(V R), i=1,2$, test statistics that follow.

Definition S.4. Define $k^{*}(x):=k(x / b)$, and let $k^{* \prime}(x)$ denote the first derivative of 
$k^{*}(x)$ from the left and $k^{* \prime \prime}(x)$ denote the second derivative of $k^{*}(x)$. We then define the following:

$$
\begin{aligned}
& \breve{F}_{i, m}(r):=F_{i, m}(r) F_{i, m}(r)^{\prime} \\
& B_{1,0}^{\delta_{m}}:=\left[W_{1}(1), W_{3}(1)\right]^{\prime}, \quad B_{1,1}^{\delta_{m}}:=(1 / 2)\left[\int_{0}^{1} V_{\bar{\alpha}_{1}, 1}(s) d s, \int_{0}^{1} V_{\bar{\alpha}_{1}, 3}(s) d s\right]^{\prime} \\
& B_{2,0}^{\delta_{m}}:=W_{2}(1), \quad B_{2,1}^{\delta_{m}}:=\int_{0}^{1} V_{\bar{\alpha}_{2}}(s) d s, \quad P_{i, j}^{\delta_{m}}:=\left[\int_{0}^{1} F_{i, m}(r) F_{i, m}(r)^{\prime} d r\right]^{-1} B_{i, j}^{\delta_{m}} \\
& W^{1}(r):=\left(W_{1}(r), W_{3}(r)\right), W^{2}(r):=W_{2}(r), \\
& V^{1}(r):=\left[\int_{0}^{r} V_{\bar{\alpha}_{1}, 1}(s) d s, \int_{0}^{r} V_{\bar{\alpha}_{1}, 3}(s) d s\right] \\
& V^{2}(r):=\int_{0}^{r} V_{\bar{\alpha}_{2}}(s) d s, \quad \hat{V}^{i}(r, \lambda):=V^{i}(r)-\breve{F}_{i, m s}(r, \lambda)^{\prime} P_{i, 1}^{\delta_{m s}}(\lambda) \\
& Q_{i, 0}^{\delta_{m}}(r):=\left[W^{i}(r)-\left[\int_{0}^{r} \breve{F}_{i, m}(s) d s\right] P_{i, 0}^{\delta_{m}}\right], \quad Q_{i, 1}^{\delta_{m}}(r):=\left[V^{i}(r)-\left[\int_{0}^{r} \breve{F}_{i, m}(s) d s\right] P_{i, 1}^{\delta_{m}}\right] \\
& \Psi_{i}^{\delta_{m}}:=\left[\int_{0}^{1} F_{i, m}(r) F_{i, m}(r)^{\prime} d r\right]^{-1}\left[\int_{0}^{1} \tilde{F}_{i, m}(r) d r\right], \\
& \Phi_{i, j}\left(b, k^{*}(\cdot)\right):=\int_{0}^{1} \int_{0}^{1}-k^{* \prime \prime}(r-s) Q_{i, j}^{\delta_{m}}(r)^{\prime} Q_{i, j}^{\delta_{m}}(r) d r d s \\
& F_{i}(r):=\left(F_{i, m}(r)^{\prime}, F_{i, m s}(r)^{\prime}\right)^{\prime}, \breve{F}_{i, m s}(r)=F_{i, m s}(r) F_{i, m}(r)^{\prime} \\
& \tilde{F}_{i, m s}(r, \lambda):=F_{i, m s}(r, \lambda)-\left[\int_{0}^{1} F_{i, m s}(r, \lambda) F_{i, m}(r)^{\prime} d r\right]\left[\int_{0}^{1} F_{i, m}(r) F_{i, m}(r)^{\prime} d r\right]^{-1} F_{i, m}(r) \\
& B_{1,0}^{\delta_{m s}}:=\left[W_{1}(1), W_{3}(1), W_{1}(1)-W_{1}(\lambda), W_{3}(1)-W_{3}(\lambda)\right]^{\prime} \\
& B_{1,1}^{\delta_{m s}}:=(1 / 2)\left[\int_{0}^{1} V_{\bar{\alpha}_{1}, 1}(s) d s, \int_{0}^{1} V_{\bar{\alpha}_{1}, 3}(s) d s\right. \\
& \left.\int_{0}^{1} V_{\bar{\alpha}_{1}, 1}(s) d s-\int_{0}^{\lambda} V_{\bar{\alpha}_{1}, 1}(s) d s, \int_{0}^{1} V_{\bar{\alpha}_{1}, 3}(s) d s-\int_{0}^{\lambda} V_{\bar{\alpha}_{1}, 3}(s) d s\right]^{\prime} \\
& B_{2,0}^{\delta_{m s}}:=\left[W_{2}(1), W_{2}(1)-W_{2}(\lambda)\right]^{\prime}, \quad B_{2,1}^{\delta_{m s}}:=\left[\int_{0}^{1} V_{\bar{\alpha}_{2}}(s) d s, \int_{0}^{1} V_{\bar{\alpha}_{2}}(s) d s-\int_{0}^{\lambda} V_{\bar{\alpha}_{2}}(s) d s\right]^{\prime} \\
& B_{i, j}:=\left(B_{i, j}^{\delta_{m}^{\prime}} B_{i, 0}^{\delta_{m s}^{\prime}}\right)^{\prime}, P_{i, j}^{\delta_{m s}}(\lambda):=R_{i}\left[\int_{0}^{1} F_{i}(r, \lambda) F_{i}(r, \lambda)^{\prime} d r\right]^{-1} B_{i, j} \\
& Q_{i, 0}^{\delta_{m s}}(r, \lambda):=\left[W^{i}(r)-\left[\int_{0}^{r} \breve{F}_{i, m s}(s, \lambda) d s\right] P_{i, 0}^{\delta_{m s}}(\lambda)\right]
\end{aligned}
$$




$$
\begin{aligned}
& Q_{i, 1}^{\delta_{m s}}(r, \lambda):=\left[V^{i}(r)-\left[\int_{0}^{r} \breve{F}_{i, m s}(s, \lambda) d s\right] P_{i, 1}^{\delta_{m s}}(\lambda)\right] \\
& \Psi_{i}^{\delta_{m s}}\left(\lambda, \lambda_{0}\right):=\left[\int_{0}^{1} \tilde{F}_{i, m s}(r, \lambda) \tilde{F}_{i, m s}(r, \lambda)^{\prime} d r\right]^{-1}\left[\int_{0}^{1} \tilde{F}_{i, m s}(r, \lambda) \tilde{F}_{i, m s}\left(r, \lambda_{0}\right)^{\prime} d r\right] \\
& \Delta_{i}\left(r, \lambda, \lambda_{0}, \kappa_{\omega_{i}, m s}\right):=\left\{\left[\int_{0}^{r} \breve{F}_{i, m s}(r, \lambda)^{\prime} d s\right] \Psi_{i}^{\delta_{m s}}\left(\lambda, \lambda_{0}\right)-\left[\int_{0}^{r} \breve{F}_{i, m s}\left(r, \lambda_{0}\right)^{\prime} d s\right]\right\} \kappa_{\omega_{i}, m s} \\
& Q_{\kappa_{\omega_{i}, m s}, i, 0}^{\delta_{m s}}\left(r, \lambda, \lambda_{0}, \kappa_{\omega_{i}, m s}\right):=\left[Q_{i, 0}^{\delta_{m s}}(r, \lambda)-\Delta_{i}\left(r, \lambda, \lambda_{0}, \kappa_{\omega_{i}, m s}\right)\right] \\
& Q_{\kappa_{\omega_{i}, m s}, i, 1}^{\delta_{m s}}\left(r, \lambda, \lambda_{0}, \kappa_{\omega_{i}, m s}\right):=\left[Q_{i, 1}^{\delta_{m s}}(r, \lambda)-\Delta_{i}\left(r, \lambda, \lambda_{0}, \kappa_{\omega_{i}, m s}\right)\right] \\
& \Phi_{\kappa_{\omega_{i}, m s}, i, j}\left(b, k^{*}(\cdot), \lambda, \lambda_{0}, \kappa_{\omega_{i}, m s}\right):=\int_{0}^{1} \int_{0}^{1}-k^{* \prime \prime}(r-s) Q_{\kappa_{\omega_{i}, m s}, i, j}^{\delta_{m s}}\left(r, \lambda, \lambda_{0}, \kappa_{\omega_{i}, m s}\right) \\
& \times Q_{\kappa_{\omega_{i}, m s}, i, j}^{\delta_{m s}}\left(r, \lambda, \lambda_{0}, \kappa_{\omega_{i}, m s}\right)^{\prime} d r d s \\
& V R_{\omega_{2}, m s, \infty}^{\kappa_{\omega_{2}, m s}}\left(\lambda, \lambda_{0}\right):=\frac{\int_{0}^{1} Q_{\kappa_{\omega_{2}, m s}, 2,1}^{\delta_{m s}}\left(r, \lambda, \lambda_{0}, \kappa_{\omega_{2}, m s}\right)^{2} d r}{\int_{0}^{1}\left(\hat{V}^{2}(r, \lambda)+\check{F}_{2, m s}(r, \lambda)^{\prime}\left[I-\Psi_{2}^{\delta_{m s}}\left(\lambda, \lambda_{0}\right)\right] \kappa_{\omega_{2}, m s}\right)^{2} d r} \\
& V R_{\omega_{1}, m s, \infty}^{\kappa_{\omega_{1}, m s}}\left(\lambda, \lambda_{0}\right):= \\
& \text { trace }\left[\int_{0}^{1}\left(\hat{V}^{1}(r, \lambda)+\check{F}_{1, m s}(r, \lambda)^{\prime}\left[I-\Psi_{1}^{\delta_{m s}}\left(\lambda, \lambda_{0}\right)\right] \kappa_{\omega_{1}, m s}\right)\right. \\
& \left.\times\left(\hat{V}^{1}(r, \lambda)+\check{F}_{1, m s}(r, \lambda)^{\prime}\left[I-\Psi_{1}^{\delta_{m s}}\left(\lambda, \lambda_{0}\right)\right] \kappa_{\omega_{1}, m s}\right)^{\prime} d r\right]^{-1} \\
& \times \int_{0}^{1} Q_{\kappa_{\omega_{1}, m s}, 1,1}^{\delta_{m s}}\left(r, \lambda, \lambda_{0}, \kappa_{\omega_{1}, m s}\right) Q_{\kappa_{\omega_{1}, m s}, 1,1}^{\delta_{m s}}\left(r, \lambda, \lambda_{0}, \kappa_{\omega_{1}, m s}\right)^{\prime} d r .
\end{aligned}
$$

\section{S.2.2. Limiting Distributions of the Mean Only Seasonal Unit Root Test Statistics}

Before detailing the limiting distributions of the robust $W_{\omega_{i}, m}(U R)$ test statistics for spectral means outlined in section 3 we will first need to establish the large sample properties of the seasonal unit root test statistics detailed in section 3.4 for use in their construction. Recall from section 3.4 that these statistics need to be such that they converge in probability to zero when the data are $I_{\omega_{i}}(0)$ at the frequency under test, and have limiting null distributions which are free of nuisance parameters (other than the local-to-unity parameter) when the data are $I_{\omega_{i}}(1)$. That these properties hold has already been established for the HEGY and variance ratio statistics in, among others, del Barrio Castro, Osborn and Taylor (2012), and Taylor (2005), respectively. For the $J_{\omega_{1}}$ and $J_{\omega_{2}}$ statistics the proofs of these results are simple extensions of the results in Vogelsang (1998) and are omitted in the interests of brevity. For reference purposes, we detail the form of the limiting distributions of these statistics when the data are $I_{\omega_{i}}(1)$ at the frequency under test in Theorem S.1. 
Theorem S.1. Let the filtered series $x_{i, t}$ be generated according to (3.4) and let Assumption 2.1 hold. Then under the local alternatives given in Definition $S .1$ and as $T \rightarrow \infty$ :

(a) if the data are $I_{\omega_{1}}(1)$ :

$$
\begin{aligned}
& V R_{\omega_{1}, m} \stackrel{d}{\rightarrow} \frac{\int_{0}^{1}\left[\int_{0}^{r} \hat{V}_{c, 1}(s) d s\right]^{2} d r}{\int_{0}^{1} \hat{V}_{c, 1}(r)^{2} d r}+\frac{\int_{0}^{1}\left[\int_{0}^{r} \hat{V}_{c, 3}(s) d s\right]^{2} d r}{\int_{0}^{1} \hat{V}_{c, 3}(r)^{2} d r} \\
&\left|F_{3,4}\right|^{-1} \stackrel{d}{\rightarrow}\left[\frac{\left(\int_{0}^{1} \hat{V}_{c, 1}(r) d \hat{V}_{c, 1}(r)+\int_{0}^{1} \hat{V}_{c, 3}(r) d \hat{V}_{c, 3}(r)\right)^{2}}{2\left(\int_{0}^{1} \hat{V}_{c, 1}(r)^{2} d r+\int_{0}^{1} \hat{V}_{c, 3}(r)^{2} d r\right)}\right. \\
&\left.+\frac{\left(\int_{0}^{1} \hat{V}_{c, 1}(r) d \hat{V}_{c, 3}(r)-\int_{0}^{1} \hat{V}_{c, 3}(r) d \hat{V}_{c, 1}(r)\right)^{2}}{2\left(\int_{0}^{1} \hat{V}_{c, 1}(r)^{2} d r+\int_{0}^{1} \hat{V}_{c, 3}(r)^{2} d r\right)}\right]^{-1} \\
& J_{\omega_{1}} \stackrel{d}{\rightarrow} \frac{\int_{0}^{1} \hat{V}_{c, 1}(r)^{2} d r+\hat{V}_{c, 3}(r)^{2} d r}{\int_{0}^{1} \tilde{V}_{c, 1}(r)^{2} d r+\tilde{V}_{c, 3}(r)^{2} d r}-1
\end{aligned}
$$

(b) if the data are $I_{\omega_{2}}(1)$ :

$$
\begin{gathered}
V R_{\omega_{2}, m} \stackrel{d}{\rightarrow} \frac{\int_{0}^{1}\left[\int_{0}^{r} \hat{V}_{c, 2}(s) d s\right]^{2} d r}{\int_{0}^{1} \hat{V}_{c, 2}(r)^{2} d r},\left|t_{2}\right|^{-1} \stackrel{d}{\rightarrow}\left|\frac{\int_{0}^{1} \hat{V}_{c, 2}(r) d \hat{V}_{c, 2}(r)}{\int_{0}^{1} \hat{V}_{c, 2}(r)^{2} d r}\right|^{-1} \\
J_{\omega_{2}} \stackrel{d}{\rightarrow} \frac{\int_{0}^{1} \hat{V}_{c, 2}(r)^{2} d r}{\int_{0}^{1} \tilde{V}_{c, 2}(r)^{2} d r}-1 .
\end{gathered}
$$

In the expressions above $\hat{V}_{c, k}(r):=V_{c, k}(r)-\int_{0}^{1} V_{c, k}(s) d s, k=1,2,3$, where $V_{c, k}(r):=$ $\int_{0}^{r} e^{-\bar{\alpha}_{i}(r-s)} d W_{k}(s), i=1,2,3$, are (mutually independent) standard Ornstein-Uhlenbeck processes with $W_{k}(r), k=1,2,3$, a set of mutually independent standard Brownian motion processes. Moreover, $\tilde{V}_{c, 2}(r)$ denotes the residuals from the projection of $V_{c, 2}(r)$ onto the space spanned by $\left(1, r, r^{2}, \ldots, r^{9}\right)^{\prime}$ and $\tilde{V}_{c, k}(r), k=1,3$ denotes the residuals from the projection of $V_{c, k}(r)$ onto the space spanned by $\left(1, r, r^{2}, \ldots, r^{8}\right)^{\prime}$.

\section{S.2.3. Limiting Distributions of Annual Frequency Spectral Mean Statistics}

We are now in a position to detail the limiting distributions of the $W_{\omega_{1}, m}(U R)$ statistics. In Theorem S.2 we first present the asymptotic distributions of the $W_{\omega_{1}, m}(U R)$ statistics when the data are $I_{\omega_{1}}(0)$.

TheOREM S.2. Let the conditions of Theorem S.1 hold and, for tests utilising a kernel based long run variance estimator, let Assumption 3.1 additionally hold. Then, as $T \rightarrow$ $\infty$, if the data are $I_{\omega_{1}}(0)$ : 
(a)

$$
\begin{aligned}
W_{\omega_{1}, m, V} & \stackrel{d}{\rightarrow}\left[P_{1,0}^{\delta_{m}}+\Psi_{1}^{\delta_{m}} \kappa_{\omega_{1}, m}\right]^{\prime}\left[\int_{0}^{1} Q_{1,0}^{\delta_{m}}(r) Q_{1,0}^{\delta_{m}}(r)^{\prime} d r\left[\int_{0}^{1} F_{1, m}(r) F_{1, m}(r)^{\prime} d r\right]^{-1}\right]^{-1} \\
& \times\left[P_{1,0}^{\delta_{m}}+\Psi_{1}^{\delta_{m}} \kappa_{\omega_{1}, m}\right]:=W_{\omega_{1}, m, V, I(0)}^{\infty}\left(\kappa_{\omega_{1}, m}\right) \\
W_{\omega_{1}, m, B V} & \stackrel{d}{\rightarrow}\left[P_{1,0}^{\delta_{m}}+\Psi_{1}^{\delta_{m}} \kappa_{\omega_{1}, m}\right]^{\prime}\left[\Phi_{1,0}^{\delta_{m}}\left(b, k^{*}(\cdot)\right)\left[\int_{0}^{1} F_{1, m}(r) F_{1, m}(r)^{\prime} d r\right]^{-1}\right]^{-1} \\
& \times\left[P_{1,0}^{\delta_{m}}+\Psi_{1}^{\delta_{m}} \kappa_{\omega_{1}, m}\right]:=W_{\omega_{1}, m, B V, I(0)}^{\infty}\left(b, k^{*}(\cdot), \kappa_{\omega_{1}, m}\right)
\end{aligned}
$$

(b)

$$
\begin{aligned}
W_{\omega_{1}, m, V}(U R) \stackrel{d}{\rightarrow} W_{\omega_{1}, m, V, I(0)}^{\infty}\left(\kappa_{\omega_{1}, m}\right) \\
W_{\omega_{1}, m, B V}(U R) \stackrel{d}{\rightarrow} W_{\omega_{1}, m, B V, I(0)}^{\infty}\left(b, k^{*}(\cdot), \kappa_{\omega_{1}, m}\right)
\end{aligned}
$$

where $P_{1,0}^{\delta_{m}}, \Psi_{1}^{\delta_{m}}, Q_{1,0}^{\delta_{m}}(r), \Phi_{1,0}^{\delta_{m}}\left(b, k^{*}(\cdot)\right)$ and $F_{1, m}(r)$ are defined in Definition S.4.

In Theorem S.3 we now give the analogue of the results in Theorem S.2 in the case where the data are $I_{\omega_{1}}(1)$.

Theorem S.3. Let the conditions of Theorem S.2 hold. Then as $T \rightarrow \infty$, if the data are $I_{\omega_{1}}(1)$ :

(a)

$$
\begin{aligned}
W_{\omega_{1}, m, V} & \stackrel{d}{\rightarrow}\left[P_{1,1}^{\delta_{m}}+\Psi_{1}^{\delta_{m}} \kappa_{\omega_{1}, m}\right]^{\prime}\left[\int_{0}^{1} Q_{1,1}^{\delta_{m}}(r) Q_{1,1}^{\delta_{m}}(r)^{\prime} d r\left[\int_{0}^{1} F_{1, m}(r) F_{1, m}(r)^{\prime} d r\right]^{-1}\right]^{-1} \\
& \times\left[P_{1,1}^{\delta_{m}}+\Psi_{1}^{\delta_{m}} \kappa_{\omega_{1}, m}\right]:=W_{\omega_{1}, m, V, I(1)}^{\infty}\left(\kappa_{\omega_{1}, m}\right) \\
W_{\omega_{1}, m, B V} & \stackrel{d}{\rightarrow}\left[P_{1,1}^{\delta_{m}}+\Psi_{1}^{\delta_{m}} \kappa_{\omega_{1}, m}\right]^{\prime}\left[\Phi_{1,1}^{\delta_{m}}\left(b, k^{*}(\cdot)\right)\left[\int_{0}^{1} F_{1, m}(r) F_{1, m}(r)^{\prime} d r\right]^{-1}\right]^{-1} \\
& \times\left[P_{1,1}^{\delta_{m}}+\Psi_{1}^{\delta_{m}} \kappa_{\omega_{1}, m}\right]:=W_{\omega_{1}, m, B V, I(1)}^{\infty}\left(b, k^{*}(\cdot), \kappa_{\omega_{1}, m}\right)
\end{aligned}
$$

(b)

$$
\begin{aligned}
& W_{\omega_{1}, m, V}(U R) \stackrel{d}{\rightarrow}\left[W_{\omega_{1}, m, V, I(1)}^{\infty}\left(\kappa_{\omega_{1}, m}\right)\right] \exp \left(-c_{\omega_{1}, m, V}^{U R} U R_{\omega_{1}, m}^{*}\right) \\
& W_{\omega_{1}, m, B V}(U R) \stackrel{d}{\rightarrow}\left[W_{\omega_{1}, m, B V, I(1)}^{\infty}\left(b, k^{*}(\cdot), \kappa_{\omega_{1}, m}\right)\right] \exp \left(-c_{\omega_{1}, m, B V}^{U R} U R_{\omega_{1}, m}^{*}\right.
\end{aligned}
$$

where $P_{1,1}^{\delta_{m}}, \Psi_{1}^{\delta_{m}}, Q_{1,1}^{\delta_{m}}(r), \Phi_{1,1}^{\delta_{m}}\left(b, k^{*}(\cdot)\right)$ and $F_{1, m}(r)$ are defined in Definition $S .4$, and where $U R_{\omega_{1}, m}^{*}$ is used to denote the limiting distribution from Theorem $S .1$ of the seasonal unit root statistic used in the construction of the statistic when the data are $I_{\omega_{1}}(1)$. 
S.2.4. Limiting Distributions of the Nyquist Frequency Spectral Mean Statistics

In this section we detail the asymptotic distribution of the Nyquist frequency $W_{\omega_{2}, m}(U R)$ test statistics. In Theorem S.4 we first present results for the case where the data are $I_{\omega_{2}}(0)$. In Theorem S.5 we then present the corresponding results for the case where the data are $I_{\omega_{2}}(1)$.

Theorem S.4. Let the conditions of Theorem S.2 hold. Then as $T \rightarrow \infty$, if the data are $I_{\omega_{2}}(0)$ :

(a)

$$
\begin{aligned}
W_{\omega_{2}, m, V} & \stackrel{d}{\rightarrow}\left[P_{2,0}^{\delta_{m}}+\Psi_{2}^{\delta_{m}} \kappa_{\omega_{2}, m}\right]^{\prime}\left[\int_{0}^{1} Q_{2,0}^{\delta_{m}}(r) Q_{2,0}^{\delta_{m}}(r)^{\prime} d r\left[\int_{0}^{1} F_{2, m}(r) F_{2, m}(r)^{\prime} d r\right]^{-1}\right]^{-1} \\
& \times\left[P_{2,0}^{\delta_{m}}+\Psi_{2}^{\delta_{m}} \kappa_{\omega_{2}, m}\right]:=W_{\omega_{2}, m, V, I(0)}^{\infty}\left(\kappa_{\omega_{2}, m}\right) \\
W_{\omega_{2}, m, B V} & \stackrel{d}{\rightarrow}\left[P_{2,0}^{\delta_{m}}+\Psi_{2}^{\delta_{m}} \kappa_{\omega_{2}, m}\right]^{\prime}\left[\Phi_{2,0}^{\delta_{m}}\left(b, k^{*}(\cdot)\right)\left[\int_{0}^{1} F_{2, m}(r) F_{2, m}(r)^{\prime} d r\right]^{-1}\right]^{-1} \\
& \times\left[P_{2,0}^{\delta_{m}}+\Psi_{2}^{\delta_{m}} \kappa_{\omega_{2}, m}\right]:=W_{\omega_{2}, m, B V, I(0)}^{\infty}\left(b, k^{*}(\cdot), \kappa_{\omega_{2}, m}\right)
\end{aligned}
$$

(b)

$$
\begin{gathered}
W_{\omega_{2}, m, V}(U R) \stackrel{d}{\rightarrow} W_{\omega_{2}, m, V, I(0)}^{\infty}\left(\kappa_{\omega_{2}, m}\right) \\
W_{\omega_{2}, m, B V}(U R) \stackrel{d}{\rightarrow} W_{\omega_{2}, m, B V, I(0)}^{\infty}\left(b, k^{*}(\cdot), \kappa_{\omega_{2}, m}\right)
\end{gathered}
$$

where $P_{2,0}^{\delta_{m}}, \Psi_{2}^{\delta_{m}}, Q_{2,0}^{\delta_{m}}(r), \Phi_{2,0}^{\delta_{m}}\left(b, k^{*}(\cdot)\right)$ and $F_{2, m}(r)$ are defined in Definition S.4.

Theorem S.5. Let the conditions of Theorem S.2 hold. Then as $T \rightarrow \infty$, if the data are $I_{\omega_{2}}(1)$ :

(a)

$$
\begin{aligned}
& W_{\omega_{2}, m, V} \stackrel{d}{\rightarrow}\left[P_{2,1}^{\delta_{m}}+\Psi_{2}^{\delta_{m}} \kappa_{\omega_{2}, m}\right]^{\prime}\left[\int_{0}^{1} Q_{2,1}^{\delta_{m}}(r) Q_{2,1}^{\delta_{m}}(r)^{\prime} d r\left[\int_{0}^{1} F_{2, m}(r) F_{2, m}(r)^{\prime} d r\right]^{-1}\right]^{-1} \\
& \times\left[P_{2,1}^{\delta_{m}}+\Psi_{2}^{\delta_{m}} \kappa_{\omega_{2}, m}\right]:=W_{\omega_{2}, m, V, I(1)}^{\infty}\left(\kappa_{\omega_{2}, m}\right) \\
& W_{\omega_{2}, m, B V} \stackrel{d}{\rightarrow}\left[P_{2,1}^{\delta_{m}}+\Psi_{2}^{\delta_{m}} \kappa_{\omega_{2}, m}\right]^{\prime}\left[\Phi_{2,1}^{\delta_{m}}\left(b, k^{*}(\cdot)\right)\left[\int_{0}^{1} F_{2, m}(r) F_{2, m}(r)^{\prime} d r\right]^{-1}\right]^{-1} \\
& \times\left[P_{2,1}^{\delta_{m}}+\Psi_{2}^{\delta_{m}} \kappa_{\omega_{2}, m}\right]:=W_{\omega_{2}, m, B V, I(1)}^{\infty}\left(b, k^{*}(\cdot), \kappa_{\omega_{2}, m}\right)
\end{aligned}
$$

(b)

$$
\begin{aligned}
W_{\omega_{2}, m, V}(U R) \stackrel{d}{\rightarrow}\left[W_{\omega_{2}, m, V, I(1)}^{\infty}\left(\kappa_{\omega_{2}, m}\right)\right] \exp \left(-c_{\omega_{2}, m, V}^{U R} U R_{\omega_{2}, m}^{*}\right) \\
W_{\omega_{2}, m, B V}(U R) \stackrel{d}{\rightarrow}\left[W_{\omega_{2}, m, B V, I(1)}^{\infty}\left(b, k^{*}(\cdot), \kappa_{\omega_{2}, m}\right)\right] \exp \left(-c_{\omega_{2}, m, B V}^{U R} U R_{\omega_{2}, m}^{*}\right)
\end{aligned}
$$


where $P_{2,1}^{\delta_{m}}, \Psi_{2}^{\delta_{m}}, Q_{2,1}^{\delta_{m}}(r), \Phi_{2,1}^{\delta_{m}}\left(b, k^{*}(\cdot)\right)$ and $F_{2, m}(r)$ are defined in Definition $S .4$ and where $U R_{\omega_{2}, m}^{*}$ is used to denote the limiting distribution from Theorem $S .1$ of the seasonal unit root test statistic used in the construction of the statistic when the data are $I_{\omega_{2}}(1)$.

\section{S.2.5. Limiting Distributions of Spectral Mean Shift Unit Root Statistics}

Before detailing the limiting distribution of the $S u p W_{\omega_{1}, m s}(V R)$ and $S u p W_{\omega_{2}, m s}(V R)$ statistics for spectral means shifts at the annual and Nyquist frequencies, respectively, we first establish in Theorem S.6 the large sample properties of the $V R_{\omega_{i}, m s}^{*}, i=1,2$, seasonal unit root statistics used in their construction.

THEOREM S.6. Let the filtered series $x_{i, t}$ be generated according to (3.4) and let Assumption 2.1 hold. Then under the local alternatives given in Definition $S .2$ and as $T \rightarrow \infty$ :

(a) (i) If the data are $I_{\omega_{1}}(0)$, then, $V R_{\omega_{1}, m s}^{*} \stackrel{p}{\rightarrow} 0$, (ii) If the data are $I_{\omega_{2}}(0)$, then, $V R_{\omega_{2}, m s}^{*} \stackrel{p}{\rightarrow} 0$;

(b) (i) If the data are $I_{\omega_{1}}(1)$, then $V R_{\omega_{1}, m s}^{*} \stackrel{d}{\rightarrow}$ inf $f_{\lambda \in\left[\lambda^{*}, 1-\lambda^{*}\right]} V R_{\omega_{1}, m s, \infty}^{\kappa_{\omega_{1}, m s}}\left(\lambda, \lambda_{0}\right):=V R_{\omega_{1}, m s}^{*, \infty}$,

(ii) If the data are $I_{\omega_{2}}(1)$, then $V R_{\omega_{2}, m s}^{*} \stackrel{d}{\rightarrow}$ inf $f_{\lambda \in\left[\lambda^{*}, 1-\lambda^{*}\right]} V R_{\omega_{2}, m s, \infty}^{\kappa_{\omega_{2}, m s}}\left(\lambda, \lambda_{0}\right):=V R_{\omega_{2}, m s}^{*, \infty}$, where $V R_{\omega_{1}, m s, \infty}^{\kappa_{\omega_{1}, m s}}\left(\lambda, \lambda_{0}\right)$ and $V R_{\omega_{2}, m s, \infty}^{\kappa_{\omega_{2}, m s}}\left(\lambda, \lambda_{0}\right)$ are defined in Definition S.4.

REMARK S.2. From the definitions of $V R_{\omega_{1}, m s, \infty}^{\kappa_{\omega_{1}, m s}}\left(\lambda, \lambda_{0}\right)$ and $V R_{\omega_{2}, m s, \infty}^{\kappa_{\omega_{1}, m s}}\left(\lambda, \lambda_{0}\right)$ in Definition $S .4$ it can be seen that the limiting distributions of the seasonal variance ratio unit root scaling statistics $V R_{\omega_{1}, m s}^{*}$ and $V R_{\omega_{2}, m s}^{*}$ depend on the seasonal level break magnitude parameters $\kappa_{\omega_{1}, m s}$ and $\kappa_{\omega_{2}, m s}$, respectively, and on the putative level break location, $\lambda_{0}$. However, these break magnitude parameters are zero under the respective null hypotheses relevant for the $S u p W_{\omega_{1}, m s}(V R)$ and $S u p W_{\omega_{2}, m s}(V R)$ statistics, while the terms involving $\lambda_{0}$ drop out of the limiting functionals, and so the large sample conditions that need to hold for the unit root scaling statistics are satisfied.

We can now proceed to establishing the limiting distributions of the $S u p W_{\omega_{i}, m s}(V R)$ $i=1,2$, spectral mean shift test statistics.

\section{S.2.6. Limiting Distributions of Annual Frequency Spectral Mean Shift Statistics}

In Theorem S.7 we first detail the limiting distribution of the annual frequency mean shift statistics when the data are $I_{\omega_{1}}(0)$. Corresponding results for when the data are $I_{\omega_{1}}(1)$ are then given in Theorem S.8.

THEOREM S.7. Let the conditions of Theorem S.6 and, for tests utilising a kernel based long run variance estimator, let Assumption 3.1 additionally hold. Then, as $T \rightarrow \infty$, if the data are $I_{\omega_{1}}(0)$ : 
(a)

$$
\begin{aligned}
W_{\omega_{1}, m s, V} \stackrel{d}{\rightarrow}\left[P_{1,0}^{\delta_{m s}}(\lambda)+\Psi_{1}^{\delta_{m s}}\left(\lambda, \lambda_{0}\right) \kappa_{\omega_{1}, m s}\right]^{\prime} \\
\times \quad\left[\int_{0}^{1} Q_{\kappa_{\omega_{1}, m s}, 1,0}^{\delta_{m s}}\left(r, \lambda, \lambda_{0}, \kappa_{\omega_{1}, m s}\right) Q_{\kappa_{\omega_{1}, m s}, 1,0}^{\delta_{m s}}\left(r, \lambda, \lambda_{0}, \kappa_{\omega_{1}, m s}\right)^{\prime} d r\right. \\
\left.\times \quad\left[\int_{0}^{1} \tilde{F}_{1, m s}(r, \lambda) \tilde{F}_{1, m s}(r, \lambda)^{\prime} d r\right]^{-1}\right]^{-1}\left[P_{1,0}^{\delta_{m s}}(\lambda)+\Psi_{1}^{\delta_{m s}}\left(\lambda, \lambda_{0}\right) \kappa_{\omega_{1}, m s}\right] \\
:=\quad W_{\omega_{1}, m s, V, I(0)}^{\infty}\left(\lambda, \lambda_{0}, \kappa_{\omega_{1}, m s}\right) \\
W_{\omega_{1}, m s, B V} \stackrel{d}{\rightarrow}\left[P_{1,0}^{\delta_{m s}}(\lambda)+\Psi_{1}^{\delta_{m s}}\left(\lambda, \lambda_{0}\right) \kappa_{\omega_{1}, m s}\right]^{\prime} \\
\times \quad\left[\Phi_{\kappa_{\omega_{1}, m s}, 1,0}\left(b, k^{*}(\cdot), \lambda, \lambda_{0}, \kappa_{\omega_{1}, m s}\right)\left[\int_{0}^{1} \tilde{F}_{1, m s}(r, \lambda) \tilde{F}_{1, m s}(r, \lambda)^{\prime} d r\right]^{-1}\right]^{-1} \\
\times \\
\quad\left[P_{1,0}^{\delta_{m s}}(\lambda)+\Psi_{1}^{\delta_{m s}}\left(\lambda, \lambda_{0}\right) \kappa_{\omega_{1}, m s}\right] \\
W_{\omega_{1}, m s, B V, I(0)}^{\infty}\left(\lambda, \lambda_{0}, b, k^{*}(\cdot), \kappa_{\omega_{1}, m s}\right) \\
:=
\end{aligned}
$$

(b)

$$
\begin{aligned}
\operatorname{Sup} W_{\omega_{1}, m s, V}(V R) \stackrel{d}{\rightarrow} \sup _{\lambda \in\left[\lambda^{*}, 1-\lambda^{*}\right]} W_{\omega_{1}, m s, V, I(0)}^{\infty}\left(\lambda, \lambda_{0}, \kappa_{\omega_{1}, m s}\right) \\
\operatorname{Sup}_{\omega_{1}, m s, B V}(V R) \stackrel{d}{\rightarrow} \sup _{\lambda \in\left[\lambda^{*}, 1-\lambda^{*}\right]} W_{\omega_{1}, m s, B V, I(0)}^{\infty}\left(\lambda, \lambda_{0}, b, k^{*}(\cdot), \kappa_{\omega_{1}, m s}\right)
\end{aligned}
$$

where $P_{1,0}^{\delta_{m s}}(\lambda), \Psi_{1}^{\delta_{m s}}\left(\lambda, \lambda_{0}\right), Q_{\kappa_{\omega_{1}, m s}, 1,0}^{\delta_{m s}}\left(r, \lambda, \lambda_{0}, \kappa_{\omega_{1}, m s}\right), \Phi_{\kappa_{\omega_{1}, m s}, 1,0}\left(b, k^{*}(\cdot), \lambda, \lambda_{0}, \kappa_{\omega_{1}, m s}\right)$ and $\tilde{F}_{1, m s}(r, \lambda)$ are defined in Definition $S .4$.

Theorem S.8. Let the conditions of Theorem S.7 hold. Then, as $T \rightarrow \infty$, if the data are $I_{\omega_{1}}(1)$ :

(a)

$$
\begin{aligned}
& W_{\omega_{1}, m s, V} \stackrel{d}{\rightarrow}\left[P_{1,1}^{\delta_{m s}}(\lambda)+\Psi_{1}^{\delta_{m s}}\left(\lambda, \lambda_{0}\right) \kappa_{\omega_{1}, m s}\right]^{\prime} \\
& \times {\left[\int_{0}^{1} Q_{\kappa_{\omega_{1}, m s}, 1,1}^{\delta_{m s}}\left(r, \lambda, \lambda_{0}, \kappa_{\omega_{1}, m s}\right) Q_{\kappa_{\omega_{1}, m s}, 1,1}^{\delta_{m_{s}}}\left(r, \lambda, \lambda_{0}, \kappa_{\omega_{1}, m s}\right)^{\prime} d r\right.} \\
& \times {\left.\left[\int_{0}^{1} \tilde{F}_{1, m s}(r, \lambda) \tilde{F}_{1, m s}(r, \lambda)^{\prime} d r\right]^{-1}\right]^{-1}\left[P_{1,1}^{\delta_{m s}}(\lambda)+\Psi_{1}^{\delta_{m s}}\left(\lambda, \lambda_{0}\right) \kappa_{\omega_{1}, m s}\right] } \\
&:=W_{\omega_{1}, m s, V, I(1)}^{\infty}\left(\lambda, \lambda_{0}, \kappa_{\omega_{1}, m s}\right) \\
& W_{\omega_{1}, m s, B V} \stackrel{d}{\rightarrow}\left[P_{1,1}^{\delta_{m s}}(\lambda)+\Psi_{1}^{\delta_{m s}}\left(\lambda, \lambda_{0}\right) \kappa_{\omega_{1}, m s}\right]^{\prime}\left[\Phi_{\kappa_{\omega_{1}, m s}, 1,1}\left(b, k^{*}(\cdot), \lambda, \lambda_{0}, \kappa_{\omega_{1}, m s}\right)\right. \\
&\left.\times\left[\int_{0}^{1} \tilde{F}_{1, m s}(r, \lambda) \tilde{F}_{1, m s}(r, \lambda)^{\prime} d r\right]^{-1}\right]^{-1} \times\left[P_{1,1}^{\delta_{m s}}(\lambda)+\Psi_{1}^{\delta_{m s}}\left(\lambda, \lambda_{0}\right) \kappa_{\omega_{1}, m s}\right] \\
&:=W_{\omega_{1}, m s, B V, I(1)}^{\infty}\left(\lambda, \lambda_{0}, b, k^{*}(\cdot), \kappa_{\omega_{1}, m s}\right)
\end{aligned}
$$


(b)

$$
\begin{aligned}
& \operatorname{Sup} W_{\omega_{1}, m s, V}(V R) \stackrel{d}{\rightarrow}\left[\sup _{\lambda \in\left[\lambda^{*}, 1-\lambda^{*}\right]} W_{\omega_{1}, m s, V, I(1)}^{\infty}\left(\lambda, \lambda_{0}, \kappa_{\omega_{1}, m s}\right)\right] \\
& \times \exp \left(-c_{\omega_{1}, m s, V}^{V R} V R_{\omega_{1}, m s, \infty}^{*}\right) \\
& \operatorname{Sup} W_{\omega_{1}, m s, B V}(V R) \stackrel{d}{\rightarrow}\left[\sup _{\lambda \in\left[\lambda^{*}, 1-\lambda^{*}\right]} W_{\omega_{1}, m s, B V, I(0)}^{\infty}\left(\lambda, \lambda_{0}, b, k^{*}(\cdot), \kappa_{\omega_{1}, m s}\right)\right] \\
& \times \exp \left(-c_{\omega_{1}, m s, B V}^{V R} V R_{\omega_{1}, m s}^{*}\right)
\end{aligned}
$$

where $P_{1,1}^{\delta_{m s}}(\lambda), \Psi_{1}^{\delta_{m s}}\left(\lambda, \lambda_{0}\right), Q_{\kappa_{\omega_{1}, m s}, 1,1}^{\delta_{m_{s}}}\left(r, \lambda, \lambda_{0}, \kappa_{\omega_{1}, m s}\right), \Phi_{\kappa_{\omega_{1}, m s}, 1,1}\left(b, k^{*}(\cdot), \lambda, \lambda_{0}, \kappa_{\omega_{1}, m s}\right)$ and $\tilde{F}_{1, m s}(r, \lambda)$ are defined in Definition $S .4$.

\section{S.2.7. Limiting Distributions of Nyquist Frequency Spectral Mean Shift Statistics}

In Theorem S.9 we first detail the limiting distribution of the Nyquist frequency mean shift statistics when the data are $I_{\omega_{2}}(0)$. The corresponding results when the data are $I_{\omega_{2}}(1)$ are then given in Theorem S.10.

Theorem S.9. Let the conditions of Theorem S.7 hold. Then, as $T \rightarrow \infty$, if the data are $I_{\omega_{2}}(0)$ :

(a)

$$
\begin{aligned}
W_{\omega_{2}, m s, V} & \stackrel{d}{\rightarrow}\left[P_{2,0}^{\delta_{m s}}(\lambda)+\Psi_{2}^{\delta_{m s}}\left(\lambda, \lambda_{0}\right) \kappa_{\omega_{2}, m s}\right]^{\prime} \\
\times & {\left[\int_{0}^{1} Q_{\kappa_{\omega_{2}, m s}, 2,0}^{\delta_{m s}}\left(r, \lambda, \lambda_{0}, \kappa_{\omega_{2}, m s}\right) Q_{\kappa_{\omega_{2}, m s}, 2,0}^{\delta_{m s}}\left(r, \lambda, \lambda_{0}, \kappa_{\omega_{2}, m s}\right)^{\prime} d r\right.} \\
\times & {\left.\left[\int_{0}^{1} \tilde{F}_{2, m s}(r, \lambda) \tilde{F}_{2, m s}(r, \lambda)^{\prime} d r\right]^{-1}\right]^{-1}\left[P_{2,0}^{\delta_{m s}}(\lambda)+\Psi_{2}^{\delta_{m s}}\left(\lambda, \lambda_{0}\right) \kappa_{\omega_{2}, m s}\right] } \\
:= & W_{\omega_{2}, m s, V, I(0)}^{\infty}\left(\lambda, \lambda_{0}, \kappa_{\omega_{2}, m s}\right) \\
W_{\omega_{2}, m s, B V} & \stackrel{d}{\rightarrow}\left[P_{2,0}^{\delta_{m s}}(\lambda)+\Psi_{2}^{\delta_{m s}}\left(\lambda, \lambda_{0}\right) \kappa_{\omega_{2}, m s}\right]^{\prime}\left[\Phi_{\kappa_{\omega_{2}, m s}, 2,0}\left(b, k^{*}(\cdot), \lambda, \lambda_{0}, \kappa_{\omega_{2}, m s}\right)\right. \\
& \left.\times\left[\int_{0}^{1} \tilde{F}_{2, m s}(r, \lambda) \tilde{F}_{2, m s}(r, \lambda)^{\prime} d r\right]^{-1}\right]^{-1} \\
& \times\left[P_{2,0}^{\delta_{m s}}(\lambda)+\Psi_{2}^{\delta_{m s}}\left(\lambda, \lambda_{0}\right) \kappa_{\omega_{2}, m s}\right] \\
& :=W_{\omega_{2}, m s, B V, I(0)}^{\infty}\left(\lambda, \lambda_{0}, b, k^{*}(\cdot), \kappa_{\omega_{2}, m s}\right)
\end{aligned}
$$

(b)

$$
\begin{aligned}
\operatorname{Sup} W_{\omega_{2}, m s, V}(V R) \stackrel{d}{\rightarrow} \sup _{\lambda \in\left[\lambda^{*}, 1-\lambda^{*}\right]} W_{\omega_{2}, m s, V, I(0)}^{\infty}\left(\lambda, \lambda_{0}, \kappa_{\omega_{2}, m s}\right) \\
\operatorname{Sup}_{\omega_{2}, m s, B V}(V R) \stackrel{d}{\rightarrow} \sup _{\lambda \in\left[\lambda^{*}, 1-\lambda^{*}\right]} W_{\omega_{2}, m s, B V, I(0)}^{\infty}\left(\lambda, \lambda_{0}, b, k^{*}(\cdot), \kappa_{\omega_{2}, m s}\right)
\end{aligned}
$$

where $P_{2,0}^{\delta_{m s}}(\lambda), \Psi_{2}^{\delta_{m s}}\left(\lambda, \lambda_{0}\right), Q_{\kappa_{\omega_{2}, m s}, 2,0}^{\delta_{m s}}\left(r, \lambda, \lambda_{0}, \kappa_{\omega_{2}, m s}\right), \Phi_{\kappa_{\omega_{2}, m s}, 2,0}\left(b, k^{*}(\cdot), \lambda, \lambda_{0}, \kappa_{\omega_{2}, m s}\right)$ and $\tilde{F}_{2, m s}(r, \lambda)$ are defined in Definition $S .4$. 
Theorem S.10. Let the conditions of Theorem S.7 hold. Then, as $T \rightarrow \infty$, if the data are $I_{\omega_{2}}(1)$ :

(a)

$$
\begin{aligned}
W_{\omega_{2}, m s, V} & \stackrel{d}{\rightarrow}\left[P_{2,1}^{\delta_{m s}}(\lambda)+\Psi_{2}^{\delta_{m s}}\left(\lambda, \lambda_{0}\right) \kappa_{\omega_{2}, m s}\right]^{\prime} \\
& \times\left[\int_{0}^{1} Q_{\kappa_{\omega_{2}, m s}, 2,1}^{\delta_{m s}}\left(r, \lambda, \lambda_{0}, \kappa_{\omega_{2}, m s}\right) Q_{\kappa_{\omega_{2}, m s}, 2,1}^{\delta_{m s}}\left(r, \lambda, \lambda_{0}, \kappa_{\omega_{2}, m s}\right)^{\prime} d r\right. \\
& \left.\times\left[\int_{0}^{1} \tilde{F}_{2, m s}(r, \lambda) \tilde{F}_{2, m s}(r, \lambda)^{\prime} d r\right]^{-1}\right]^{-1}\left[P_{2,1}^{\delta_{m s}}(\lambda)+\Psi_{2}^{\delta_{m s}}\left(\lambda, \lambda_{0}\right) \kappa_{\omega_{2}, m s}\right] \\
& :=W_{\omega_{2}, m s, V, I(1)}^{\infty}\left(\lambda, \lambda_{0}, \kappa_{\omega_{2}, m s}\right) \\
W_{\omega_{2}, m s, B V} & \stackrel{d}{\rightarrow}\left[P_{2,1}^{\delta_{m s}}(\lambda)+\Psi_{2}^{\delta_{m s}}\left(\lambda, \lambda_{0}\right) \kappa_{\omega_{2}, m s}\right]^{\prime}\left[\Phi_{\kappa_{\omega_{2}, m s}, 2,1}\left(b, k^{*}(\cdot), \lambda, \lambda_{0}, \kappa_{\omega_{2}, m s}\right)\right. \\
& \left.\times\left[\int_{0}^{1} \tilde{F}_{2, m s}(r, \lambda) \tilde{F}_{2, m s}(r, \lambda)^{\prime} d r\right]^{-1}\right]^{-1} \\
& \times\left[P_{2,1}^{\delta_{m s}}(\lambda)+\Psi_{2}^{\delta_{m s}}\left(\lambda, \lambda_{0}\right) \kappa_{\omega_{2}, m s}\right] \\
& :=W_{\omega_{2}, m s, B V, I(1)}^{\infty}\left(\lambda, \lambda_{0}, b, k^{*}(\cdot), \kappa_{\omega_{2}, m s}\right)
\end{aligned}
$$

(b)

$$
\begin{aligned}
& \operatorname{Sup} W_{\omega_{2}, m s, V}(V R) \stackrel{d}{\rightarrow}\left[\sup _{\lambda \in\left[\lambda^{*}, 1-\lambda^{*}\right]} W_{\omega_{2}, m s, V, I(1)}^{\infty}\left(\lambda, \lambda_{0}, \kappa_{\omega_{2}, m s}\right)\right] \\
& \times \exp \left(-c_{\omega_{2}, m s, V}^{V R} V R_{\omega_{2}, m s}^{*, \infty}\right) \\
& \operatorname{Sup} W_{\omega_{2}, m s, B V}(V R) \stackrel{d}{\rightarrow}\left[\sup _{\lambda \in\left[\lambda^{*}, 1-\lambda^{*}\right]} W_{\omega_{2}, m s, B V, I(0)}^{\infty}\left(\lambda, \lambda_{0}, b, k^{*}(\cdot), \kappa_{\omega_{2}, m s}\right)\right] \\
& \times \exp \left(-c_{\omega_{2}, m s, B V}^{V R} V R_{\omega_{2}, m s}^{*, \infty} *\right)
\end{aligned}
$$

where $P_{2,1}^{\delta_{m s}}(\lambda), \Psi_{2}^{\delta_{m s}}\left(\lambda, \lambda_{0}\right), Q_{\kappa_{\omega_{2}, m s}, 2,1}^{\delta_{m_{s}}}\left(r, \lambda, \lambda_{0}, \kappa_{\omega_{2}, m s}\right), \Phi_{\kappa_{\omega_{2}, m s}, 2,1}\left(b, k^{*}(\cdot), \lambda, \lambda_{0}, \kappa_{\omega_{2}, m s}\right)$ and $\tilde{F}_{2, m s}(r, \lambda)$ are defined in Definition $S .4$.

\section{S.2.8. Joint Frequency Tests}

In this section we outline the limiting null distributions of the joint frequency tests outlined in section 3.5 and discuss the choice of critical values and the scaling constants $\tau_{\omega_{m}}$ and $\tau_{\omega_{m s}}$ required to yield tests robust to the order of integration of the data at all frequencies.

S.2.8.1. Joint Tests for Seasonal Spectral Means Consider first the joint test statistic for seasonal spectral means, $J S_{\delta_{m}}(V R)$, in (3.19). The limiting null distributions of the $W_{\omega_{i}, m, B V}(V R)$ statistics $i=1,2$, when the data are $I_{\omega_{i}}(0)$ are given by (S.2.6) with $\kappa_{\omega_{1}, m}=0$ for $\omega_{i}=1$ and (S.2.14) with $\kappa_{\omega_{2}, m}=0$ for $\omega_{i}=2$. Using the independence of these limiting distributions and the CMT we therefore have that when $x_{t}$ is simultaneously $I_{\omega_{1}}(0)$ and $I_{\omega_{2}}(0)$, then

$$
J S_{\delta_{m}}(V R) \stackrel{d}{\rightarrow} \frac{1}{2} \sum_{i=1}^{2} W_{\omega_{i}, m, B V, I(0)}^{\infty}\left(b, k^{*}(\cdot), 0\right) .
$$


Critical values for the $J S_{\delta_{m}}(V R)$ test are taken from (S.2.35) with $\tau_{\delta_{m}}$ chosen to control the asymptotic size of the test when the data are, potentially, $I_{\omega_{i}}(1) i=1,2$.

S.2.8.2. Joint Tests for Spectral Mean Shifts Consider next the joint test statistic for spectral mean shifts, $J S_{\delta_{m s}}(V R)$, in (3.20). When the data are $I_{\omega_{i}}(0), i=1,2$, the limiting null distributions of the $\operatorname{Sup}_{\omega_{i}, m s, B V}(V R), i=1,2$, statistics are given by (S.2.22) with $\kappa_{\omega_{1}, m s}=0$ and (S.2.30) with $\kappa_{\omega_{2}, m s}=0$, respectively. These limiting distributions are mutually independent, so that when $x_{t}$ is simultaneously $I_{\omega_{1}}(0)$ and $I_{\omega_{2}}(0)$

$$
\begin{aligned}
J S_{\delta_{m s}}(V R) \stackrel{d}{\rightarrow}(1 / 2)\left[\sup _{\lambda \in\left[\lambda^{*}, 1-\lambda^{*}\right]} W_{\omega_{1}, m s, B V, I(0)}^{\infty}\left(\lambda, \lambda_{0}, b, k^{*}(\cdot), 0\right)\right. \\
\\
\left.+\sup _{\lambda \in\left[\lambda^{*}, 1-\lambda^{*}\right]} W_{\omega_{2}, m s, B V, I(0)}^{\infty}\left(\lambda, \lambda_{0}, b, k^{*}(\cdot), 0\right)\right] .
\end{aligned}
$$

Critical values for the $J S_{\delta_{m s}}(V R)$ test are taken from (S.2.36) with $\tau_{\delta_{m}}$ chosen to control the asymptotic size of the test when the data are, potentially, $I_{\omega_{i}}(1) i=1,2$.

\section{S.3. ASYMPTOTIC LOCAL POWER}

We now report results relating to the asymptotic local power of the tests procedures outlined in this paper using direct Monte Carlo simulation of their limiting distributions reported in Section S.2. The simulations were conducted at an asymptotic 5\% level. When reporting asymptotic local power for tests using the kernel-based variance estimate in (3.8) we present results where the bandwidth is set equal to the optimal bandwidth reported in S.1.1. We report results for the case where the data are near-integrated at the frequency under test as we will show in section S.4 that the finite sample power of the tests is reflective of their asymptotic local power in this scenario. Results for the Nyquist frequency mean shift tests outlined in the paper are not reported because the asymptotic local power of these tests is identical to that of the zero frequency mean shift tests reported in Sayginsoy and Vogelsang (2011) by virtue of their asymptotic distributions being identical. Results for tests for a Nyquist frequency mean are reported, however, as tests for a zero frequency intercept are not considered in Bunzel and Vogelsang (2005).

Figure S.1 graphs asymptotic local power functions of the Nyquist frequency mean tests when $\alpha_{2}=1-\bar{\alpha}_{2} / T$ with values of $\bar{\alpha}_{2}=0,5,10,15$; recall that $\bar{\alpha}_{2}=0$ corresponds to the case where the process contains an exact unit root at the Nyquist frequency. We observe from Figure S.1 that, for a given Nyquist frequency unit root test statistic, the $W_{\omega_{2}, m, B V}(U R)$ test has greater asymptotic local power overall than the corresponding $W_{\omega_{2}, m, V}(U R)$ test. Overall, tests based on the HEGY seasonal unit root test deliver the highest asymptotic local power among the tests considered, followed by those based on the $V R$ seasonal unit root test with the tests based on the $J$ seasonal unit root test having the lowest asymptotic local power overall. Note that as $\bar{\alpha}_{2}$ increases, tests based on the HEGY unit root test statistic begin to display a large degree of undersizing, which we will show in section S.4 leads to these tests having poor finite sample power properties when the Nyquist frequency autoregressive root is not close to unity.

Figure S.2 graphs asymptotic local power functions of the annual frequency mean tests when $\alpha_{1}=1-\bar{\alpha}_{1} / T$ with values of $\bar{\alpha}_{1}=0,5,10,15$; again, recall that $\bar{\alpha}_{1}=0$ corresponds to the case where the process contains a complex pair of exact unit roots at the annual frequency. We, again, observe that, for a given unit root statistic, the $W_{\omega_{1}, m, B V}(U R)$ tests have greater overall asymptotic local power than the $W_{\omega_{1}, m, V}(U R)$ tests regardless 
of which annual frequency unit root statistic is used in their construction. Where $\bar{\alpha}_{1}=0$, we see that tests constructed using the $V R$ seasonal unit root test statistic display the best overall power performance among the tests considered. As $\bar{\alpha}_{1}$ increases we see that the power ranking of the tests changes slightly, with tests based on the HEGY and $J$ seasonal unit root statistics showing modest power gains relative to the tests based on the $V R$ annual frequency unit root statistics. We will show in section S.4 that in finite samples this reversal in power rankings is not too drastic, and that the $W_{\omega_{1}, m, B V}(V R)$ test displays the best overall finite sample power properties.

Figure S.3 graphs asymptotic local power functions of the annual frequency mean shift tests when $\alpha_{1}=1-\bar{\alpha}_{1} / T$ with values of $\bar{\alpha}_{1}=0,5,10,15$. Much like in the case of the tests for seasonal means, we observe that the $W_{\omega_{1}, m s, B V}(V R)$ test has greater asymptotic local power overall than the $W_{\omega_{1}, m s, V}(V R)$ test. For $\bar{\alpha}_{1}=0$, the $W_{\omega_{1}, m s, B V}(V R)$ test dominates the $W_{\omega_{1}, m s, V}(V R)$ test for all local alternatives considered. We observe that the $W_{\omega_{1}, m s, B V}(V R)$ test exhibits a modest degree of undersize for $\bar{\alpha}_{1}=10$ and $\bar{\alpha}_{1}=15$. This pattern of undersize can be shown to continue for larger values of $\bar{\alpha}_{1}$, and this will be shown in section S.4 to lead to a shortfall in the power of the $W_{\omega_{1}, m s, B V}(V R)$ relative to the $W_{\omega_{1}, m s, V}(V R)$ test in finite samples when the data are stationary at the annual frequency and the break magnitude is small.

Overall, and consistent with the results in Sayginsoy and Vogelsang (2011) and Bunzel and Vogelsang (2005), we can observe that, for all of the tests we consider, asymptotic local power is, for a given seasonal unit root statistic in their formulation, greater when based on a test statistic which uses the kernel-based variance estimator in (3.8) with the optimal bandwidth rule given in S.1.1, than when using the variance estimator in (3.9). 

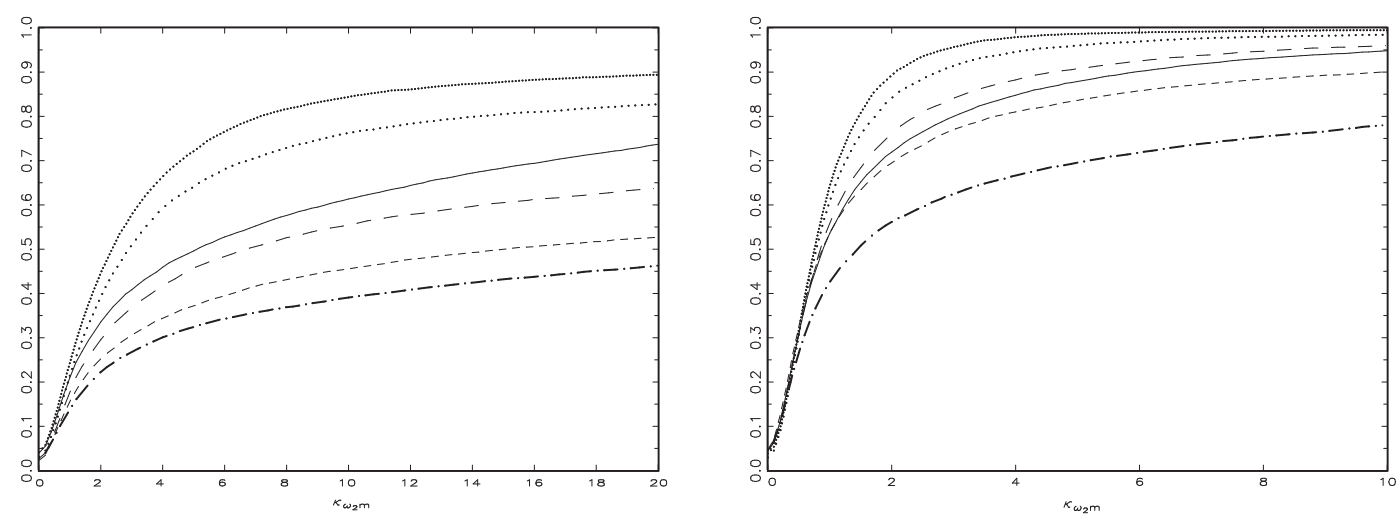

$$
\bar{\alpha}_{2}=0
$$

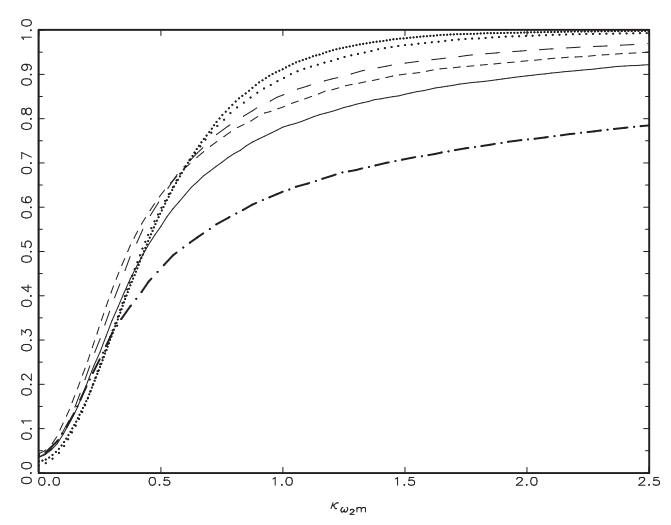

$$
\bar{\alpha}_{2}=5
$$

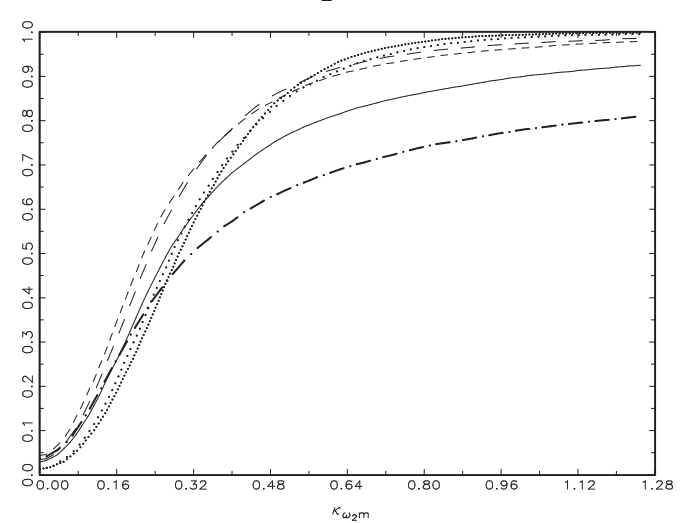

$$
\bar{\alpha}_{2}=10
$$

$\bar{\alpha}_{2}=15$

$W_{\omega_{2}, m, V}(V R):-\cdot-, W_{\omega_{2}, m, V}(H): \cdots \cdots, W_{\omega_{2}, m, V}(J): \cdots \cdots$

$W_{\omega_{2}, m, B V}(V R):-, W_{\omega_{2}, m, B V}(H): \cdots \cdot, W_{\omega_{2}, m, B V}(J):---$

Figure S.1. Asymptotic local power, Nyquist Frequency Mean Tests 

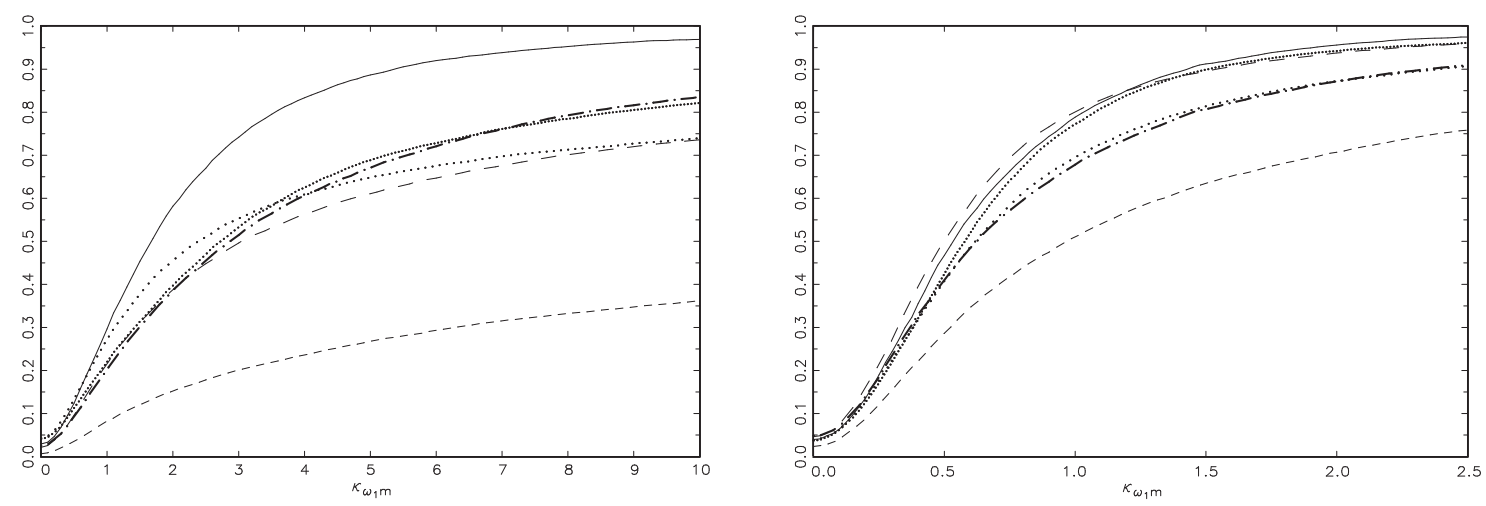

$\bar{\alpha}_{1}=0$

$\bar{\alpha}_{1}=5$
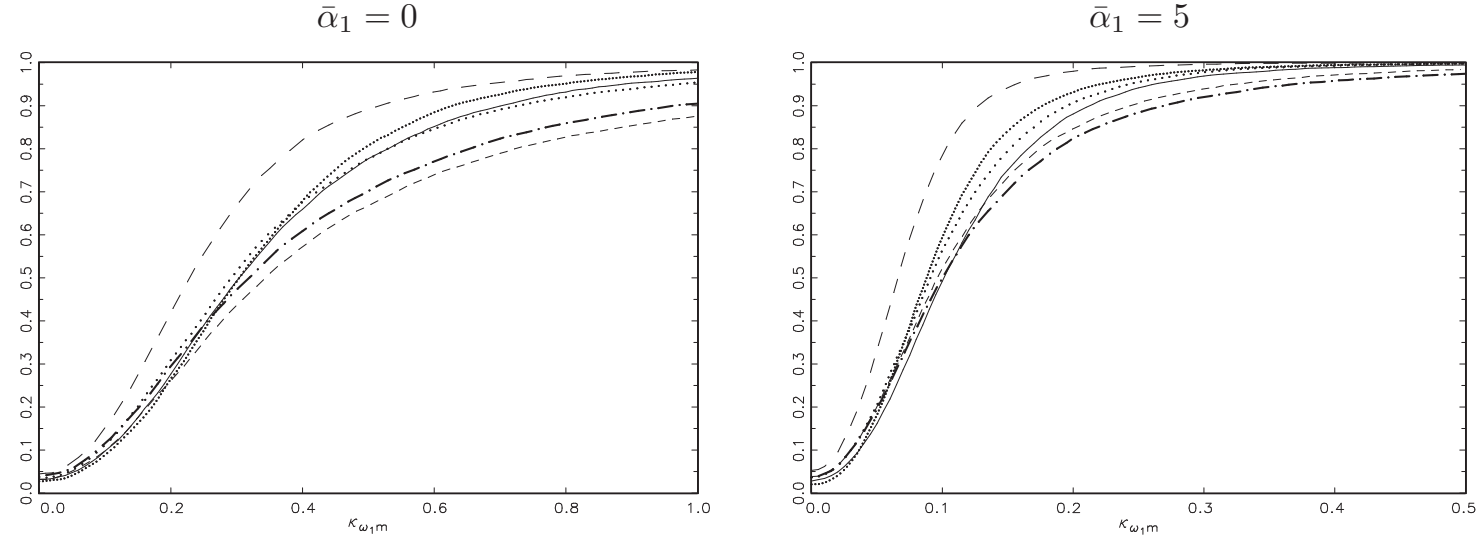

$\bar{\alpha}_{1}=10$

$\bar{\alpha}_{1}=15$

$W_{\omega_{1}, m, V}(V R):-\cdot-, W_{\omega_{1}, m, V}(H): \cdots \cdots, W_{\omega_{1}, m, V}(J):-\cdots$

$W_{\omega_{1}, m, B V}(V R):-, W_{\omega_{1}, m, B V}(H): \cdots \cdots, W_{\omega_{1}, m, B V}(J):---$

Figure S.2. Asymptotic local power, Annual Frequency Mean Tests 

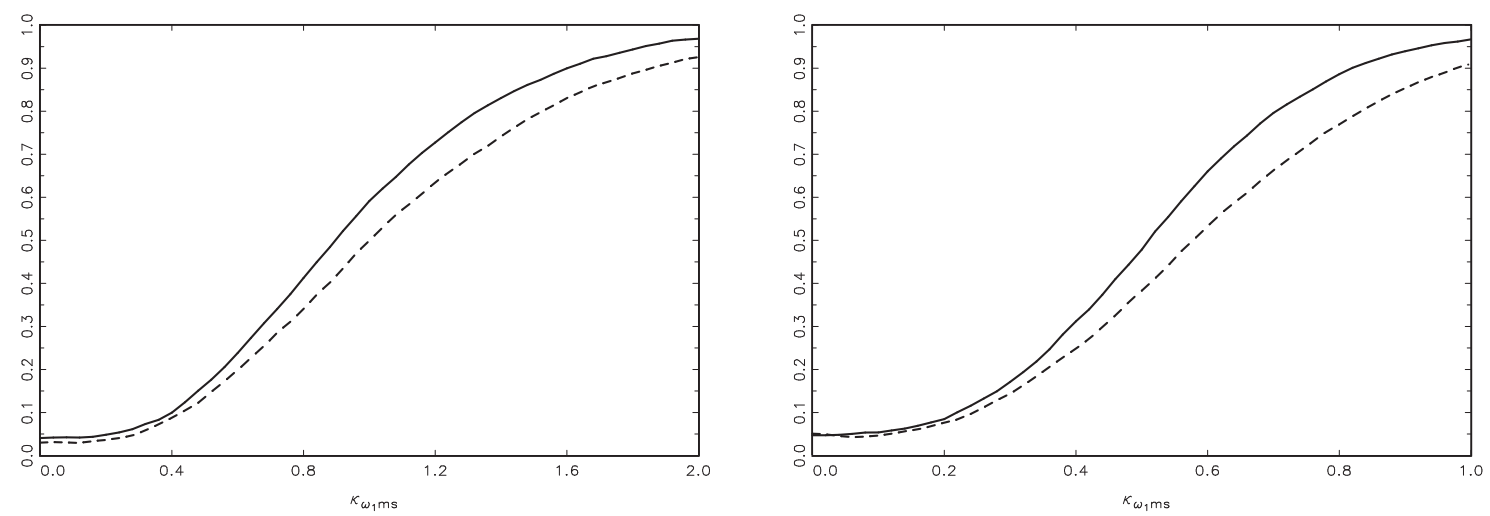

$\bar{\alpha}_{1}=0$

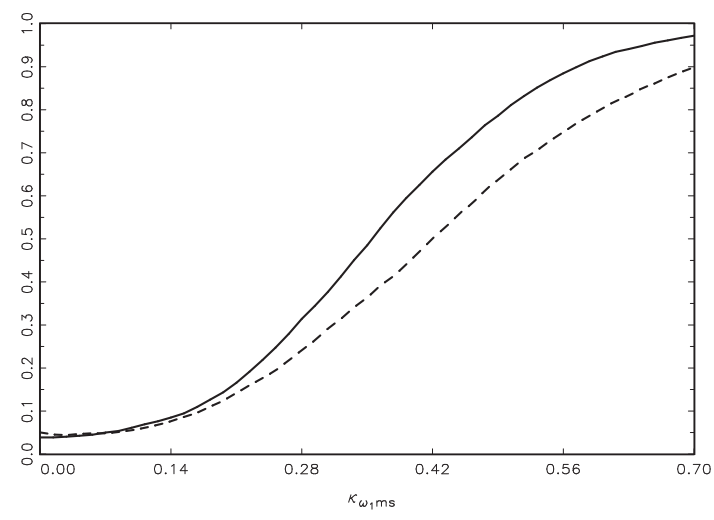

$\bar{\alpha}_{1}=5$

$\bar{\alpha}_{1}=10$

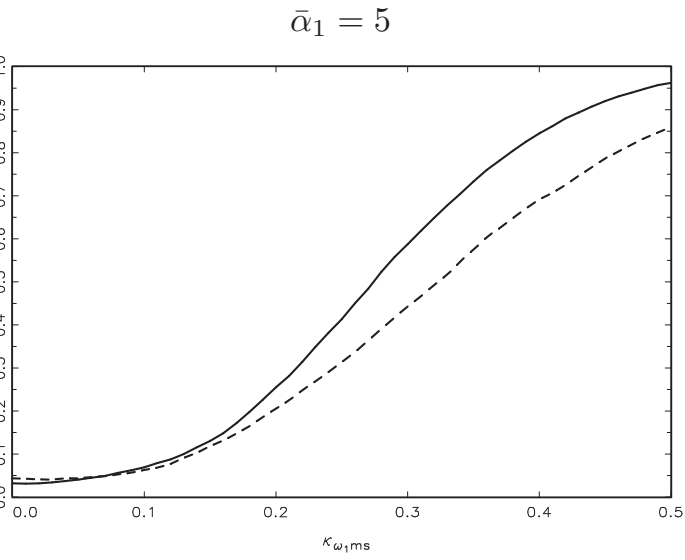

$\operatorname{Sup} W_{\omega_{2}, m s, V}(V R):-\cdots-\operatorname{Sup}_{\omega_{2}, m s, B V}(V R)$ :

$\bar{\alpha}_{1}=15$

Figure S.3. Asymptotic local power, Annual Frequency Mean Shift Tests 


\section{S.4. FINITE SAMPLE SIMULATIONS}

In this section we present results of Monte Carlo simulations performed to investigate the finite sample size and power properties of the test statistics outlined in our paper. All Monte Carlo simulations were performed in Gauss 8.0 using 10,000 replications. All tests performed using the $\tilde{\sigma}_{B V, i}^{2}$ and $\hat{\sigma}_{B V, i}^{2}, i=1,2$, variance estimators are constructed using the Daniell kernel with the bandwidth selected using the method outlined in Section 5.1.

\section{S.4.1. Individual Frequency Mean Tests}

S.4.1.1. Empirical Size We begin by assessing the finite sample empirical size properties of our proposed test statistics using data generated according to the simulation DGP:

$$
\left(1-\alpha_{0} L\right)\left(1+\alpha_{1}^{2} L^{2}\right)\left(1+\alpha_{2} L\right) x_{t}=v_{t}, t=1, \ldots, T,
$$

with $v_{t} \sim N I I D(0,1)$, setting the initial conditions $x_{-3}=\cdots=x_{0}=0$. When performing the HEGY unit root tests we choose $q$ using the familiar Schwarz (1978) information criterion with a maximum lag length $q_{\max }=4$. Results are reported for a range of $\alpha_{0}, \alpha_{1}^{2}, \alpha_{2} \in\{1.00,0.95,0.9,0.7,0.5,0.0\}$ and $T \in\{100,240\}$.

Results for the Nyquist frequency tests are reported in Tables S.7-S.8. Empirical size is close to the nominal level for most tests when $\alpha_{2}=1$, but the tests display a tendency to undersize when the process is stationary at the Nyquist frequency. The tests based on the HEGY unit root test statistic exhibit the poorest overall size control, being both oversized when $\alpha_{2}=1$ and quite severely undersized when $\alpha_{2}<1$. The test with the best overall size control is the $W_{\omega_{2}, m, B V}(V R)$ test.

Results for the annual frequency tests are reported in Tables S.9-S.10. Results here are fairly mixed, with all of the tests showing some moderate size distortions for different values of $\alpha_{1}^{2}$, but with no consistent pattern observed across all tests. A number of tests exhibit oversize for values of $\alpha_{1}^{2}$ close to unity in smaller samples, although these tests are also undersized across other values of $\alpha_{1}^{2}$. 
S. Astill and A.M.R. Taylor

Table S.7. Finite Sample Size - Nyquist Frequency Mean Tests $T=100$

\begin{tabular}{|c|c|c|c|c|c|c|c|}
\hline$\alpha_{2}$ & $\alpha_{0}=\alpha_{1}^{2}$ & $W_{\omega_{2}, m, V}(V R)$ & $W_{\omega_{2}, m, V}(H)$ & $W_{\omega_{2}, m, V}(J)$ & $W_{\omega_{2}, m, B V}(V R)$ & $W_{\omega_{2}, m, B V}(H)$ & $W_{\omega_{2}, m, B V}(J)$ \\
\hline \multirow[t]{6}{*}{1.00} & 1.00 & 0.047 & 0.064 & 0.041 & 0.057 & 0.068 & 0.043 \\
\hline & 0.95 & 0.048 & 0.064 & 0.041 & 0.056 & 0.067 & 0.043 \\
\hline & 0.90 & 0.048 & 0.064 & 0.040 & 0.056 & 0.067 & 0.042 \\
\hline & 0.70 & 0.047 & 0.063 & 0.035 & 0.054 & 0.066 & 0.039 \\
\hline & 0.50 & 0.045 & 0.061 & 0.029 & 0.051 & 0.064 & 0.034 \\
\hline & 0.00 & 0.039 & 0.058 & 0.017 & 0.044 & 0.060 & 0.020 \\
\hline \multirow[t]{6}{*}{0.95} & 1.00 & 0.055 & 0.037 & 0.054 & 0.053 & 0.036 & 0.050 \\
\hline & 0.95 & 0.055 & 0.036 & 0.054 & 0.053 & 0.036 & 0.050 \\
\hline & 0.90 & 0.055 & 0.036 & 0.053 & 0.053 & 0.037 & 0.051 \\
\hline & 0.70 & 0.053 & 0.037 & 0.048 & 0.051 & 0.039 & 0.045 \\
\hline & 0.50 & 0.050 & 0.038 & 0.043 & 0.050 & 0.041 & 0.042 \\
\hline & 0.00 & 0.047 & 0.037 & 0.029 & 0.046 & 0.045 & 0.031 \\
\hline \multirow{6}{*}{0.90} & 1.00 & 0.044 & 0.023 & 0.048 & 0.037 & 0.020 & 0.042 \\
\hline & 0.95 & 0.044 & 0.023 & 0.048 & 0.037 & 0.021 & 0.042 \\
\hline & 0.90 & 0.044 & 0.023 & 0.047 & 0.037 & 0.021 & 0.040 \\
\hline & 0.70 & 0.043 & 0.023 & 0.044 & 0.037 & 0.027 & 0.038 \\
\hline & 0.50 & 0.042 & 0.023 & 0.040 & 0.037 & 0.030 & 0.038 \\
\hline & 0.00 & 0.038 & 0.022 & 0.029 & 0.036 & 0.034 & 0.031 \\
\hline \multirow[t]{6}{*}{0.70} & 1.00 & 0.043 & 0.018 & 0.052 & 0.035 & 0.015 & 0.035 \\
\hline & 0.95 & 0.043 & 0.018 & 0.052 & 0.035 & 0.015 & 0.034 \\
\hline & 0.90 & 0.043 & 0.018 & 0.050 & 0.035 & 0.016 & 0.034 \\
\hline & 0.70 & 0.041 & 0.017 & 0.048 & 0.034 & 0.016 & 0.031 \\
\hline & 0.50 & 0.040 & 0.016 & 0.045 & 0.032 & 0.016 & 0.032 \\
\hline & 0.00 & 0.036 & 0.014 & 0.036 & 0.035 & 0.027 & 0.039 \\
\hline \multirow[t]{6}{*}{0.50} & 1.00 & 0.047 & 0.020 & 0.055 & 0.039 & 0.017 & 0.032 \\
\hline & 0.95 & 0.046 & 0.020 & 0.054 & 0.038 & 0.017 & 0.032 \\
\hline & 0.90 & 0.046 & 0.020 & 0.053 & 0.038 & 0.017 & 0.031 \\
\hline & 0.70 & 0.044 & 0.019 & 0.052 & 0.036 & 0.017 & 0.027 \\
\hline & 0.50 & 0.042 & 0.017 & 0.049 & 0.035 & 0.018 & 0.026 \\
\hline & 0.00 & 0.036 & 0.014 & 0.041 & 0.034 & 0.022 & 0.038 \\
\hline \multirow[t]{6}{*}{0.00} & 1.00 & 0.052 & 0.025 & 0.056 & 0.041 & 0.023 & 0.034 \\
\hline & 0.95 & 0.052 & 0.024 & 0.056 & 0.040 & 0.024 & 0.034 \\
\hline & 0.90 & 0.051 & 0.024 & 0.055 & 0.039 & 0.023 & 0.033 \\
\hline & 0.70 & 0.049 & 0.023 & 0.055 & 0.039 & 0.021 & 0.032 \\
\hline & 0.50 & 0.047 & 0.021 & 0.054 & 0.038 & 0.020 & 0.028 \\
\hline & 0.00 & 0.040 & 0.016 & 0.046 & 0.035 & 0.018 & 0.024 \\
\hline
\end{tabular}


Robust Tests for Deterministic Seasonality and Seasonal Mean Shifts

$\mathrm{S} 23$

Table S.8. Finite Sample Size - Nyquist Frequency Mean Tests $T=240$

\begin{tabular}{|c|c|c|c|c|c|c|c|}
\hline$\alpha_{2}$ & $\alpha_{0}=\alpha_{1}^{2}$ & $W_{\omega_{2}, m, V}(V R)$ & $W_{\omega_{2}, m, V}(H)$ & $W_{\omega_{2}, m, V}(J)$ & $W_{\omega_{2}, m, B V}(V R)$ & $W_{\omega_{2}, m, B V}(H)$ & $W_{\omega_{2}, m, B V}(J)$ \\
\hline \multirow[t]{6}{*}{1.00} & 1.00 & 0.045 & 0.055 & 0.036 & 0.049 & 0.059 & 0.038 \\
\hline & 0.95 & 0.045 & 0.054 & 0.036 & 0.049 & 0.060 & 0.038 \\
\hline & 0.90 & 0.045 & 0.054 & 0.036 & 0.049 & 0.059 & 0.038 \\
\hline & 0.70 & 0.044 & 0.054 & 0.034 & 0.049 & 0.059 & 0.037 \\
\hline & 0.50 & 0.044 & 0.054 & 0.032 & 0.048 & 0.060 & 0.033 \\
\hline & 0.00 & 0.040 & 0.053 & 0.025 & 0.044 & 0.057 & 0.025 \\
\hline \multirow[t]{6}{*}{0.95} & 1.00 & 0.045 & 0.021 & 0.048 & 0.037 & 0.017 & 0.042 \\
\hline & 0.95 & 0.045 & 0.020 & 0.048 & 0.037 & 0.018 & 0.042 \\
\hline & 0.90 & 0.044 & 0.020 & 0.047 & 0.036 & 0.019 & 0.043 \\
\hline & 0.70 & 0.044 & 0.020 & 0.045 & 0.037 & 0.022 & 0.043 \\
\hline & 0.50 & 0.043 & 0.020 & 0.044 & 0.037 & 0.026 & 0.042 \\
\hline & 0.00 & 0.040 & 0.020 & 0.040 & 0.038 & 0.031 & 0.040 \\
\hline \multirow[t]{6}{*}{0.90} & 1.00 & 0.043 & 0.017 & 0.051 & 0.033 & 0.014 & 0.037 \\
\hline & 0.95 & 0.043 & 0.017 & 0.051 & 0.033 & 0.015 & 0.037 \\
\hline & 0.90 & 0.043 & 0.016 & 0.051 & 0.034 & 0.015 & 0.038 \\
\hline & 0.70 & 0.042 & 0.016 & 0.050 & 0.035 & 0.016 & 0.037 \\
\hline & 0.50 & 0.041 & 0.016 & 0.049 & 0.036 & 0.018 & 0.041 \\
\hline & 0.00 & 0.039 & 0.015 & 0.044 & 0.038 & 0.028 & 0.046 \\
\hline \multirow[t]{6}{*}{0.70} & 1.00 & 0.049 & 0.021 & 0.057 & 0.034 & 0.018 & 0.035 \\
\hline & 0.95 & 0.049 & 0.021 & 0.057 & 0.035 & 0.018 & 0.034 \\
\hline & 0.90 & 0.049 & 0.021 & 0.056 & 0.036 & 0.018 & 0.033 \\
\hline & 0.70 & 0.048 & 0.020 & 0.056 & 0.037 & 0.018 & 0.031 \\
\hline & 0.50 & 0.047 & 0.019 & 0.055 & 0.040 & 0.018 & 0.029 \\
\hline & 0.00 & 0.043 & 0.016 & 0.051 & 0.040 & 0.020 & 0.043 \\
\hline \multirow[t]{6}{*}{0.50} & 1.00 & 0.053 & 0.025 & 0.057 & 0.037 & 0.019 & 0.036 \\
\hline & 0.95 & 0.052 & 0.025 & 0.057 & 0.036 & 0.019 & 0.035 \\
\hline & 0.90 & 0.052 & 0.025 & 0.057 & 0.036 & 0.021 & 0.035 \\
\hline & 0.70 & 0.051 & 0.024 & 0.057 & 0.036 & 0.021 & 0.035 \\
\hline & 0.50 & 0.050 & 0.022 & 0.056 & 0.040 & 0.021 & 0.031 \\
\hline & 0.00 & 0.046 & 0.018 & 0.053 & 0.040 & 0.022 & 0.033 \\
\hline \multirow[t]{6}{*}{0.00} & 1.00 & 0.054 & 0.033 & 0.056 & 0.043 & 0.021 & 0.037 \\
\hline & 0.95 & 0.054 & 0.032 & 0.056 & 0.042 & 0.022 & 0.036 \\
\hline & 0.90 & 0.054 & 0.032 & 0.056 & 0.042 & 0.022 & 0.035 \\
\hline & 0.70 & 0.054 & 0.030 & 0.056 & 0.041 & 0.022 & 0.033 \\
\hline & 0.50 & 0.053 & 0.028 & 0.056 & 0.039 & 0.020 & 0.029 \\
\hline & 0.00 & 0.049 & 0.022 & 0.055 & 0.041 & 0.022 & 0.029 \\
\hline
\end{tabular}


S. Astill and A.M.R. Taylor

Table S.9. Finite Sample Size - Annual Frequency Mean Tests $T=100$

\begin{tabular}{|c|c|c|c|c|c|c|c|}
\hline$\alpha_{1}^{2}$ & $\alpha_{0}=\alpha_{2}$ & $W_{\omega_{1}, m, V}(V R)$ & $W_{\omega_{1}, m, V}(H)$ & $W_{\omega_{1}, m, V}(J)$ & $W_{\omega_{1}, m, B V}(V R)$ & $W_{\omega_{1}, m, B V}(H)$ & $W_{\omega_{1}, m, B V}(J)$ \\
\hline \multirow[t]{6}{*}{1.00} & 1.00 & 0.041 & 0.065 & 0.011 & 0.047 & 0.063 & 0.036 \\
\hline & 0.95 & 0.042 & 0.065 & 0.010 & 0.046 & 0.062 & 0.034 \\
\hline & 0.90 & 0.041 & 0.063 & 0.009 & 0.046 & 0.060 & 0.033 \\
\hline & 0.70 & 0.039 & 0.064 & 0.008 & 0.044 & 0.052 & 0.027 \\
\hline & 0.50 & 0.037 & 0.064 & 0.006 & 0.042 & 0.047 & 0.021 \\
\hline & 0.00 & 0.034 & 0.062 & 0.003 & 0.038 & 0.041 & 0.015 \\
\hline \multirow[t]{6}{*}{0.95} & 1.00 & 0.062 & 0.060 & 0.025 & 0.056 & 0.053 & 0.062 \\
\hline & 0.95 & 0.061 & 0.060 & 0.024 & 0.055 & 0.053 & 0.059 \\
\hline & 0.90 & 0.061 & 0.061 & 0.023 & 0.054 & 0.052 & 0.056 \\
\hline & 0.70 & 0.057 & 0.060 & 0.018 & 0.051 & 0.047 & 0.046 \\
\hline & 0.50 & 0.054 & 0.057 & 0.014 & 0.049 & 0.041 & 0.037 \\
\hline & 0.00 & 0.052 & 0.056 & 0.010 & 0.044 & 0.033 & 0.026 \\
\hline \multirow[t]{6}{*}{0.90} & 1.00 & 0.055 & 0.051 & 0.025 & 0.044 & 0.029 & 0.056 \\
\hline & 0.95 & 0.055 & 0.050 & 0.024 & 0.044 & 0.029 & 0.054 \\
\hline & 0.90 & 0.054 & 0.049 & 0.024 & 0.043 & 0.030 & 0.053 \\
\hline & 0.70 & 0.053 & 0.048 & 0.020 & 0.041 & 0.031 & 0.044 \\
\hline & 0.50 & 0.050 & 0.048 & 0.017 & 0.039 & 0.028 & 0.036 \\
\hline & 0.00 & 0.047 & 0.047 & 0.013 & 0.037 & 0.025 & 0.028 \\
\hline \multirow[t]{6}{*}{0.70} & 1.00 & 0.039 & 0.037 & 0.033 & 0.023 & 0.009 & 0.043 \\
\hline & 0.95 & 0.038 & 0.036 & 0.032 & 0.023 & 0.009 & 0.041 \\
\hline & 0.90 & 0.037 & 0.036 & 0.031 & 0.023 & 0.009 & 0.040 \\
\hline & 0.70 & 0.036 & 0.035 & 0.027 & 0.021 & 0.009 & 0.037 \\
\hline & 0.50 & 0.034 & 0.033 & 0.023 & 0.020 & 0.012 & 0.030 \\
\hline & 0.00 & 0.032 & 0.032 & 0.019 & 0.018 & 0.012 & 0.024 \\
\hline \multirow[t]{6}{*}{0.50} & 1.00 & 0.034 & 0.037 & 0.037 & 0.022 & 0.014 & 0.034 \\
\hline & 0.95 & 0.034 & 0.037 & 0.036 & 0.021 & 0.013 & 0.033 \\
\hline & 0.90 & 0.033 & 0.036 & 0.035 & 0.020 & 0.012 & 0.032 \\
\hline & 0.70 & 0.032 & 0.035 & 0.032 & 0.018 & 0.008 & 0.030 \\
\hline & 0.50 & 0.030 & 0.033 & 0.029 & 0.017 & 0.007 & 0.026 \\
\hline & 0.00 & 0.029 & 0.031 & 0.025 & 0.016 & 0.007 & 0.022 \\
\hline \multirow[t]{6}{*}{0.00} & 1.00 & 0.031 & 0.034 & 0.037 & 0.008 & 0.010 & 0.010 \\
\hline & 0.95 & 0.031 & 0.033 & 0.036 & 0.009 & 0.010 & 0.010 \\
\hline & 0.90 & 0.031 & 0.033 & 0.036 & 0.011 & 0.011 & 0.011 \\
\hline & 0.70 & 0.030 & 0.033 & 0.034 & 0.014 & 0.010 & 0.016 \\
\hline & 0.50 & 0.030 & 0.033 & 0.034 & 0.016 & 0.009 & 0.014 \\
\hline & 0.00 & 0.030 & 0.034 & 0.033 & 0.012 & 0.008 & 0.016 \\
\hline
\end{tabular}


Robust Tests for Deterministic Seasonality and Seasonal Mean Shifts

$\mathrm{S} 25$

Table S.10. Finite Sample Size - Annual Frequency Mean Tests $T=240$

\begin{tabular}{|c|c|c|c|c|c|c|c|}
\hline$\alpha_{1}^{2}$ & $\alpha_{0}=\alpha_{2}$ & $W_{\omega_{1}, m, V}(V R)$ & $W_{\omega_{1}, m, V}(H)$ & $W_{\omega_{1}, m, V}(J)$ & $W_{\omega_{1}, m, B V}(V R)$ & $W_{\omega_{1}, m, B V}(H)$ & $W_{\omega_{1}, m, B V}(J)$ \\
\hline \multirow[t]{6}{*}{1.00} & 1.00 & 0.030 & 0.048 & 0.009 & 0.034 & 0.046 & 0.030 \\
\hline & 0.95 & 0.030 & 0.049 & 0.009 & 0.033 & 0.044 & 0.030 \\
\hline & 0.90 & 0.030 & 0.048 & 0.009 & 0.033 & 0.043 & 0.028 \\
\hline & 0.70 & 0.029 & 0.048 & 0.008 & 0.032 & 0.037 & 0.024 \\
\hline & 0.50 & 0.028 & 0.047 & 0.007 & 0.032 & 0.032 & 0.021 \\
\hline & 0.00 & 0.027 & 0.047 & 0.005 & 0.031 & 0.028 & 0.019 \\
\hline \multirow[t]{6}{*}{0.95} & 1.00 & 0.052 & 0.045 & 0.030 & 0.037 & 0.022 & 0.059 \\
\hline & 0.95 & 0.052 & 0.044 & 0.030 & 0.037 & 0.023 & 0.059 \\
\hline & 0.90 & 0.052 & 0.044 & 0.029 & 0.037 & 0.024 & 0.057 \\
\hline & 0.70 & 0.051 & 0.044 & 0.027 & 0.036 & 0.025 & 0.050 \\
\hline & 0.50 & 0.050 & 0.044 & 0.024 & 0.036 & 0.024 & 0.045 \\
\hline & 0.00 & 0.049 & 0.044 & 0.021 & 0.035 & 0.021 & 0.040 \\
\hline \multirow[t]{6}{*}{0.90} & 1.00 & 0.043 & 0.038 & 0.034 & 0.028 & 0.009 & 0.052 \\
\hline & 0.95 & 0.043 & 0.038 & 0.034 & 0.028 & 0.009 & 0.051 \\
\hline & 0.90 & 0.043 & 0.038 & 0.033 & 0.027 & 0.010 & 0.050 \\
\hline & 0.70 & 0.042 & 0.037 & 0.031 & 0.026 & 0.013 & 0.047 \\
\hline & 0.50 & 0.041 & 0.037 & 0.030 & 0.025 & 0.015 & 0.041 \\
\hline & 0.00 & 0.040 & 0.035 & 0.027 & 0.024 & 0.013 & 0.036 \\
\hline \multirow[t]{6}{*}{0.70} & 1.00 & 0.037 & 0.038 & 0.044 & 0.039 & 0.019 & 0.039 \\
\hline & 0.95 & 0.036 & 0.038 & 0.043 & 0.036 & 0.018 & 0.037 \\
\hline & 0.90 & 0.036 & 0.038 & 0.043 & 0.033 & 0.017 & 0.036 \\
\hline & 0.70 & 0.035 & 0.037 & 0.041 & 0.022 & 0.013 & 0.035 \\
\hline & 0.50 & 0.034 & 0.036 & 0.039 & 0.019 & 0.012 & 0.033 \\
\hline & 0.00 & 0.033 & 0.034 & 0.037 & 0.019 & 0.010 & 0.031 \\
\hline \multirow[t]{6}{*}{0.50} & 1.00 & 0.038 & 0.039 & 0.045 & 0.037 & 0.040 & 0.040 \\
\hline & 0.95 & 0.038 & 0.039 & 0.044 & 0.036 & 0.038 & 0.039 \\
\hline & 0.90 & 0.037 & 0.039 & 0.044 & 0.035 & 0.033 & 0.036 \\
\hline & 0.70 & 0.036 & 0.038 & 0.042 & 0.031 & 0.022 & 0.031 \\
\hline & 0.50 & 0.035 & 0.037 & 0.042 & 0.026 & 0.017 & 0.028 \\
\hline & 0.00 & 0.033 & 0.036 & 0.040 & 0.020 & 0.015 & 0.026 \\
\hline \multirow[t]{6}{*}{0.00} & 1.00 & 0.038 & 0.041 & 0.043 & 0.023 & 0.028 & 0.028 \\
\hline & 0.95 & 0.038 & 0.041 & 0.043 & 0.022 & 0.028 & 0.027 \\
\hline & 0.90 & 0.038 & 0.041 & 0.043 & 0.022 & 0.027 & 0.026 \\
\hline & 0.70 & 0.038 & 0.040 & 0.043 & 0.019 & 0.025 & 0.023 \\
\hline & 0.50 & 0.036 & 0.039 & 0.042 & 0.017 & 0.023 & 0.022 \\
\hline & 0.00 & 0.035 & 0.037 & 0.041 & 0.018 & 0.022 & 0.022 \\
\hline
\end{tabular}


S.4.1.2. Empirical Power We now investigate the finite sample empirical power properties of our proposed tests. To that end, data were generated according to the following simulation DGP:

$$
\begin{aligned}
x_{t} & =z_{t}^{\prime} \gamma+u_{t} \\
\left(1-\alpha_{0} L\right)\left(1+\alpha_{1}^{2} L^{2}\right)\left(1+\alpha_{2} L\right) u_{t} & =v_{t}, t=1, \ldots, T,
\end{aligned}
$$

with $v_{t} \sim \operatorname{NIID}(0,1)$, setting the initial conditions $u_{-3}=\cdots=u_{0}=0$ and where $\gamma:=\left(\gamma_{0}, \gamma_{1,1}, \gamma_{1,2}, \gamma_{2}\right)^{\prime}$.

We first examine the power of the Nyquist frequency mean tests. To do so we set $\gamma_{0}=\gamma_{1,1}=\gamma_{1,2}=0$ and examine the empirical rejection frequencies of the tests of $H_{0}: \gamma_{2}=0$ versus $H_{1}: \gamma_{2} \neq 0$ at the nominal $5 \%$ level. We set $\alpha_{0}=\alpha_{1}^{2}=1$ and $\alpha_{2} \in$ $\{0.00,0.50,0.70,0.90,0.95,1.00\}$ with a grid of 50 steps of values for $\gamma_{2}$ from to 0 to $\gamma_{2, \max }$ and with the choices of $\gamma_{2, \max } \in\{0.250,0.500,1.000,6.250,25.0,50.0\}$ corresponding to the six choices of $\alpha_{2}$, respectively. We only report results for a sample size of $T=100$ because the power ranking of all of the seasonal mean tests was found to be broadly similar for $T=240$. The results are reported in Figure S.4. It is seen from the results that no one test dominates all of the others over all of the scenarios considered. Generally, the greater the power of a test when $\alpha_{2}=1$, the more severe is the undersize of that test when $\alpha_{2}<1$, leading to a loss of power in stationary scenarios. This is consistent with the asymptotic local power of the tests detailed in Figure S.1, with for instance, the $W_{\omega_{2}, m, B V}(H)$ test dominating when $\alpha_{2}=1$, but suffering a drop off in power as $\alpha_{2}$ decreases due to the undersize exhibited by the test in stationary scenarios. The general pattern is that tests with the best power performance when the data are integrated at the Nyquist frequency exhibit lower power in DGPs where the data are stationary at the Nyquist frequency and vice versa. In all instances the power performance of the $W_{\omega_{2}, m, B V}(U R)$ test is superior to that of the $W_{\omega_{2}, m, V}(U R)$ test for any given unit root test statistic $U R$. We therefore recommend the use of the $W_{\omega_{2}, m, B V}(V R)$ test in practice because this test would appear to strike the best balance between finite sample size and power across all of the scenarios considered.

We next consider the finite sample power performance of the annual frequency mean tests. To do so we set $\gamma_{0}=\gamma_{2}=0$ and examine the empirical rejection frequencies of the tests of $H_{0}: \gamma_{1}=0$ against $H_{1}: \gamma_{1} \neq 0$ at the nominal $5 \%$ level, again for a sample size of $T=100$ now with $\alpha_{0}=\alpha_{2}=1$ and $\alpha_{1}^{2} \in\{0.00,0.50,0.70,0.90,0.95,1.00\}$. We set $\gamma_{1,1}=\gamma_{1,2}$ and consider a grid of 50 steps of values for $\gamma_{1,1}$ from to 0 to $\gamma_{1,1 \text { max }}$ with the choices of $\gamma_{1,1 \mathrm{max}} \in\{1.0,2.0,5.0,10.0,10.0,50.0\}$ corresponding to the six choices of $\alpha_{1}^{2}$, respectively. The results are reported in Figure S.5. As with the corresponding Nyquist frequency tests in Figure S.4, no one test is seen to dominate across all of the scenarios considered, with tests that perform best when the data are integrated at the annual frequency performing relatively poorly when the data are stationary at the annual frequency. Once again, tests constructed using the $\tilde{\sigma}_{B V}^{2}$ variance estimator perform better overall than those constructed using the $\tilde{\sigma}_{V}^{2}$ variance estimator. These results again tally with the asymptotic local power results given in Figure S.2, with the $W_{\omega_{1}, m, B V}(V R)$ test displaying the best power performance when $\alpha_{1}^{2}=1$, but suffering from a small shortfall in power relative to the $W_{\omega_{1}, m, B V}(J)$ test in more stationary scenarios. Given the size properties and relative power of the tests across all scenarios, we therefore recommend the use of the $W_{\omega_{1}, m, B V}(V R)$ test in practice as this test has the best overall finite sample size and power properties. 

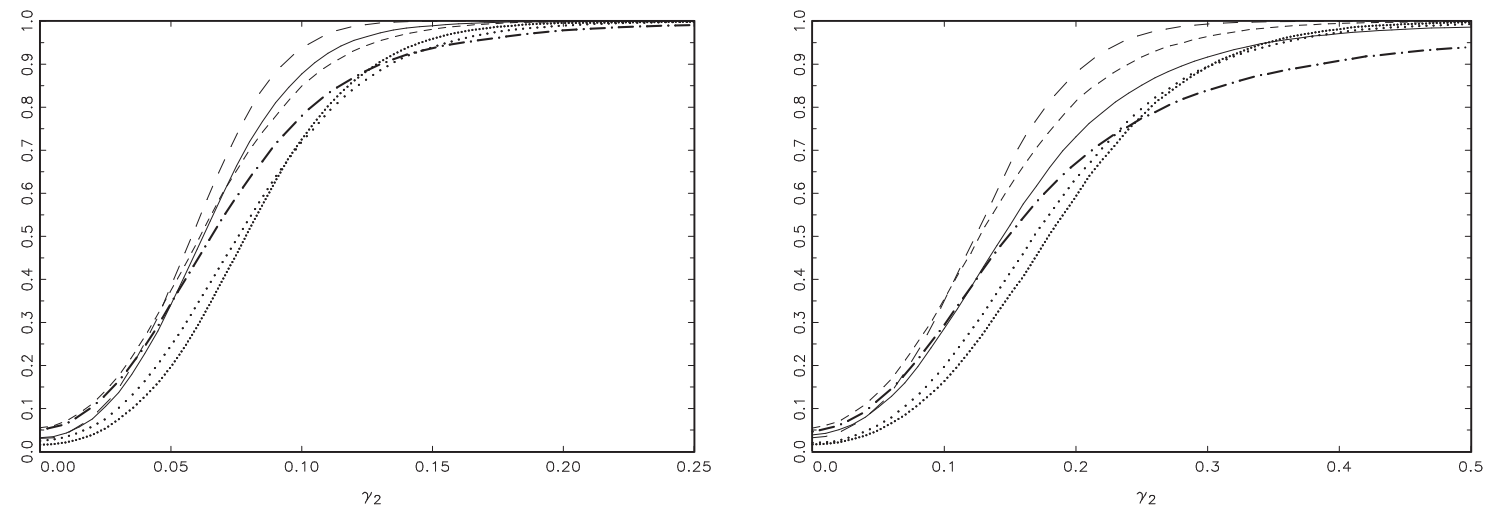

$\alpha_{2}=0.00$

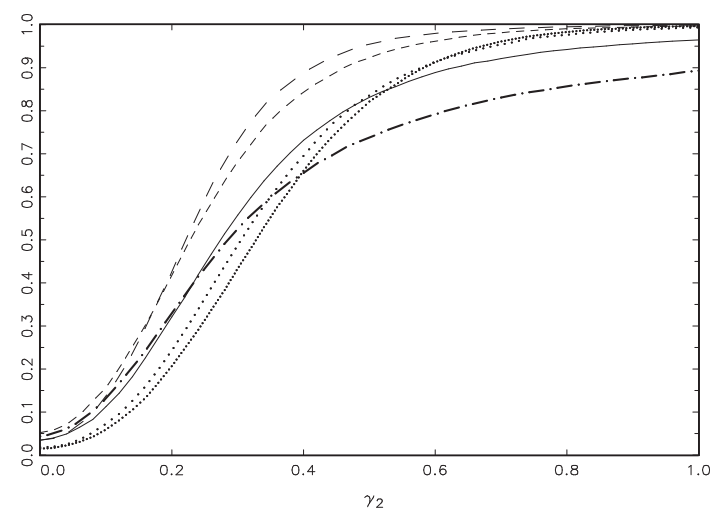

$\alpha_{2}=0.50$

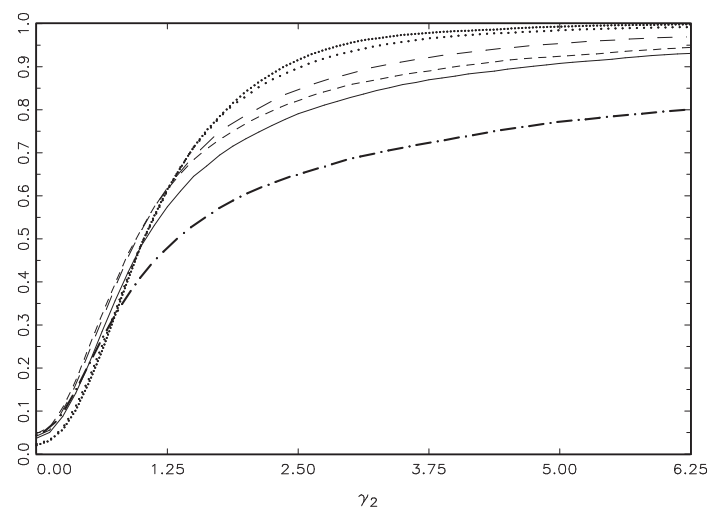

$\alpha_{2}=0.70$

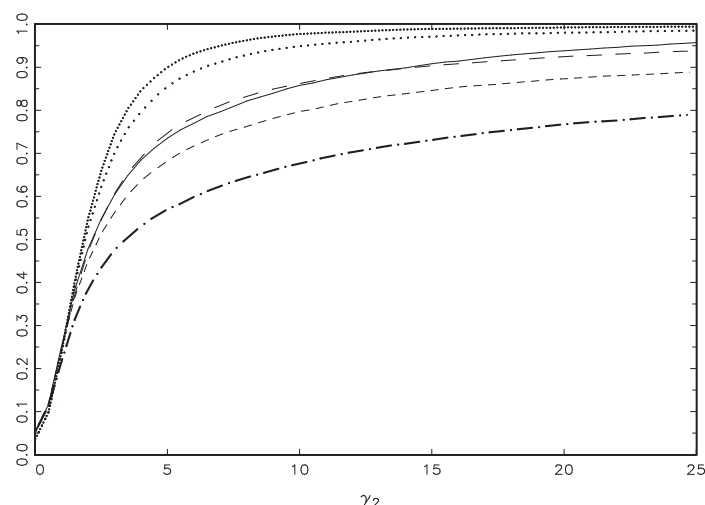

$\alpha_{2}=0.90$

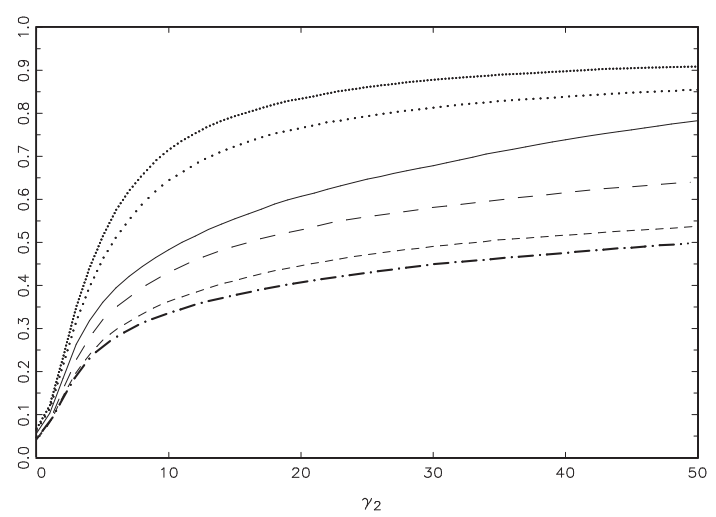

$\alpha_{2}=0.95$

$\alpha_{2}=1.00$

$W_{\omega_{2}, m, V}(V R):-\cdot-, W_{\omega_{2}, m, V}(H): \cdots \cdots, W_{\omega_{2}, m, V}(J): \cdots \cdots$

$W_{\omega_{2}, m, B V}(V R):-, W_{\omega_{2}, m, B V}(H): \cdots \cdots, W_{\omega_{2}, m, B V}(J):---$

Figure S.4. Finite Sample Power, Nyquist Frequency Mean Tests, $\alpha_{0}=\alpha_{1}^{2}=1$ 

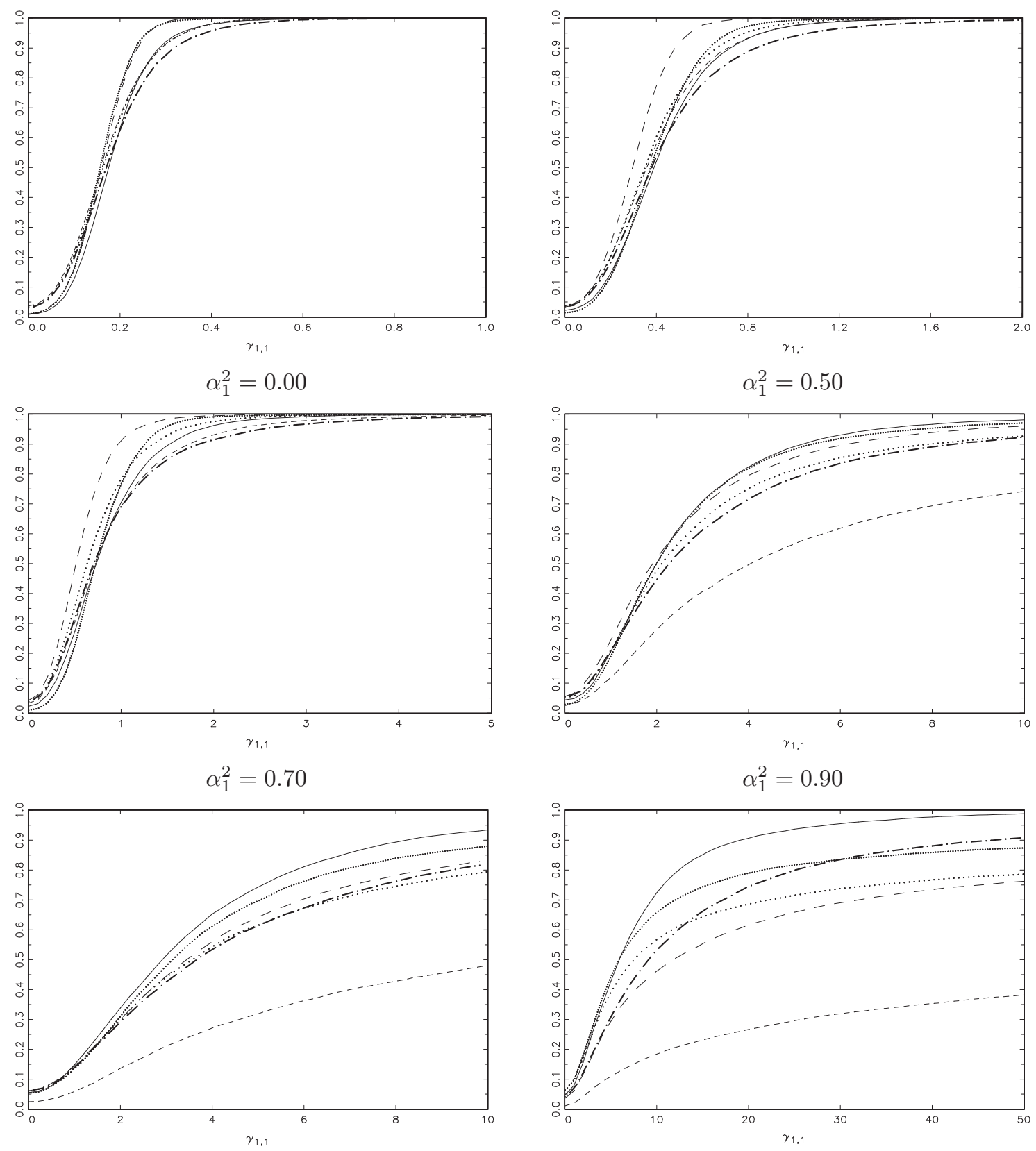

$$
\begin{gathered}
\alpha_{1}^{2}=0.95 \\
\alpha_{1}^{2}=1.00 \\
W_{\omega_{1}, m, V}(V R):-\cdot-, W_{\omega_{1}, m, V}(H): \cdots \cdots, W_{\omega_{1}, m, V}(J):-\cdots \\
W_{\omega_{1}, m, B V}(V R):- \\
\hline
\end{gathered}
$$

Figure S.5. Finite Sample Power, Annual Frequency Mean Tests, $\alpha_{0}=\alpha_{2}=1$ 


\section{S.4.2. Joint Frequency Seasonal Mean Tests}

We next present the results of a Monte Carlo simulation study to explore the finite sample size and power properties of the $J S_{\delta_{m}}(V R)$ test.

S.4.2.1. Empirical Size We begin by examining the empirical size performance of the joint-frequency $J S_{\delta_{m}}(V R)$ test under the null of no deterministic seasonality. To that end, data were simulated according to the following simulation DGP:

$$
\left(1-\alpha_{0} L\right)\left(1+\alpha_{1}^{2} L^{2}\right)\left(1+\alpha_{2} L\right) x_{t}=v_{t}, t=1, \ldots, T,
$$

with $v_{t} \sim N I I D(0,1)$ setting the initial conditions $x_{-3}=\cdots=x_{0}=0$. The $J S_{\delta_{m}}(V R)$ test was performed at an asymptotic level of $5 \%$ and the results are reported in Table S.11 for a range of $\alpha_{2} \in\{1.00,0.95,0.9,0.7,0.5,0.0\}, \alpha_{1}^{2} \in\{1.00,0.95,0.9,0.7,0.5,0.0\}$ setting $\alpha_{0}=1$. Results are reported for sample sizes of $T=\{100,240\}$. It is seen from the results that the $J S_{\delta_{m}}(V R)$ test has empirical size comparable to the individual $W_{\omega_{1}, m, B V}(V R)$ and $W_{\omega_{2}, m, B V}(V R)$ tests used in its construction, with the size of the $J S_{\delta_{m}}(V R)$ test lying close to the nominal level when the data are near-integrated at the seasonal frequencies but exhibiting some modest undersize when the data are stationary at the seasonal frequencies. Importantly, the finite sample size of the test does not exceed the nominal level across the range of scenarios considered, highlighting the robustness of the test to the order of integration of the data at both seasonal frequencies.

Table S.11. Finite Sample Size $J S_{\delta_{m}}(V R)$ Test $\alpha_{0}=1$

\begin{tabular}{rcccccc}
\multicolumn{7}{c}{$T=100$} \\
\hline$\alpha_{2}$ & 1.00 & 0.95 & 0.90 & 0.70 & 0.50 & 0.00 \\
\hline 1.00 & 0.042 & 0.048 & 0.041 & 0.022 & 0.034 & 0.029 \\
0.95 & 0.039 & 0.044 & 0.036 & 0.018 & 0.029 & 0.028 \\
0.90 & 0.038 & 0.042 & 0.035 & 0.016 & 0.022 & 0.019 \\
0.70 & 0.039 & 0.044 & 0.035 & 0.015 & 0.020 & 0.015 \\
0.50 & 0.037 & 0.044 & 0.036 & 0.015 & 0.019 & 0.014 \\
0.00 & 0.037 & 0.044 & 0.034 & 0.015 & 0.013 & 0.004 \\
\hline
\end{tabular}

\begin{tabular}{rcccccc}
\multicolumn{7}{c}{$T=240$} \\
\hline$\alpha_{2}$ & 1.00 & 0.95 & 0.90 & 0.70 & 0.50 & 0.00 \\
\hline 1.00 & 0.029 & 0.035 & 0.024 & 0.037 & 0.038 & 0.033 \\
0.95 & 0.027 & 0.033 & 0.020 & 0.031 & 0.030 & 0.025 \\
0.90 & 0.027 & 0.032 & 0.019 & 0.029 & 0.029 & 0.022 \\
0.70 & 0.028 & 0.033 & 0.020 & 0.025 & 0.027 & 0.019 \\
0.50 & 0.027 & 0.033 & 0.021 & 0.022 & 0.025 & 0.013 \\
0.00 & 0.027 & 0.031 & 0.019 & 0.029 & 0.021 & 0.002 \\
\hline
\end{tabular}

S.4.2.2. Empirical Power We next report results for the finite sample power of the $J S_{\delta_{m}}(V R)$ test against the alternative that both a Nyquist and an annual frequency mean are present in the data. We would therefore expect the empirical rejection rate of the 
$J S_{\delta_{m}}(V R)$ test to be greater than either individual $W_{\omega_{1}, m, B V}(V R)$ and $W_{\omega_{2}, m, B V}(V R)$ tests in this scenario.

Data were generated according to the following DGP:

$$
\begin{aligned}
x_{t} & =z_{t}^{\prime} \gamma+u_{t} \\
\left(1-\alpha_{0} L\right)\left(1+\alpha_{1}^{2} L^{2}\right)\left(1+\alpha_{2} L\right) u_{t} & =v_{t}, t=1, \ldots, 100,
\end{aligned}
$$

with $v_{t} \sim \operatorname{NIID}(0,1)$ setting the initial conditions $u_{-3}=\cdots=u_{0}=0$. We set $\gamma=\left(0, \lambda_{A}^{-1} g^{*}, \lambda_{A}^{-1} g *, \lambda_{N}^{-1} g^{*}\right)^{\prime}$ for a range of values of $g^{*}$ from 1 to 50 . Figure S.6 presents power curves for the $J S_{\delta_{m}}(V R), W_{\omega_{1}, m, B V}(V R)$ and $W_{\omega_{2}, m, B V}(V R)$ tests for $\alpha_{0}=1.00$ and $\alpha_{1}^{2}=\alpha_{2}=\{0.00,0.50,0.70,0.90,0.95,1.00\}$ with the values $\lambda_{A}=$ $\{50.0 .25 .0,10.0,4.0,4.0,1.0\} \quad \lambda_{N}=\{100.0,75.0,40.0,6.5,1.5,0.5\}$ corresponding to the six scenarios considered. The values of $\lambda_{A}$ and $\lambda_{N}$ are chosen such that the grid of alternatives at each frequency closely mirrors those used when examining each individual frequency. On examining the power curves, we see that across all of the scenarios considered the empirical power of the $J S_{\delta_{m}}(V R)$ test is greater overall than each of the individual tests, as anticipated. 

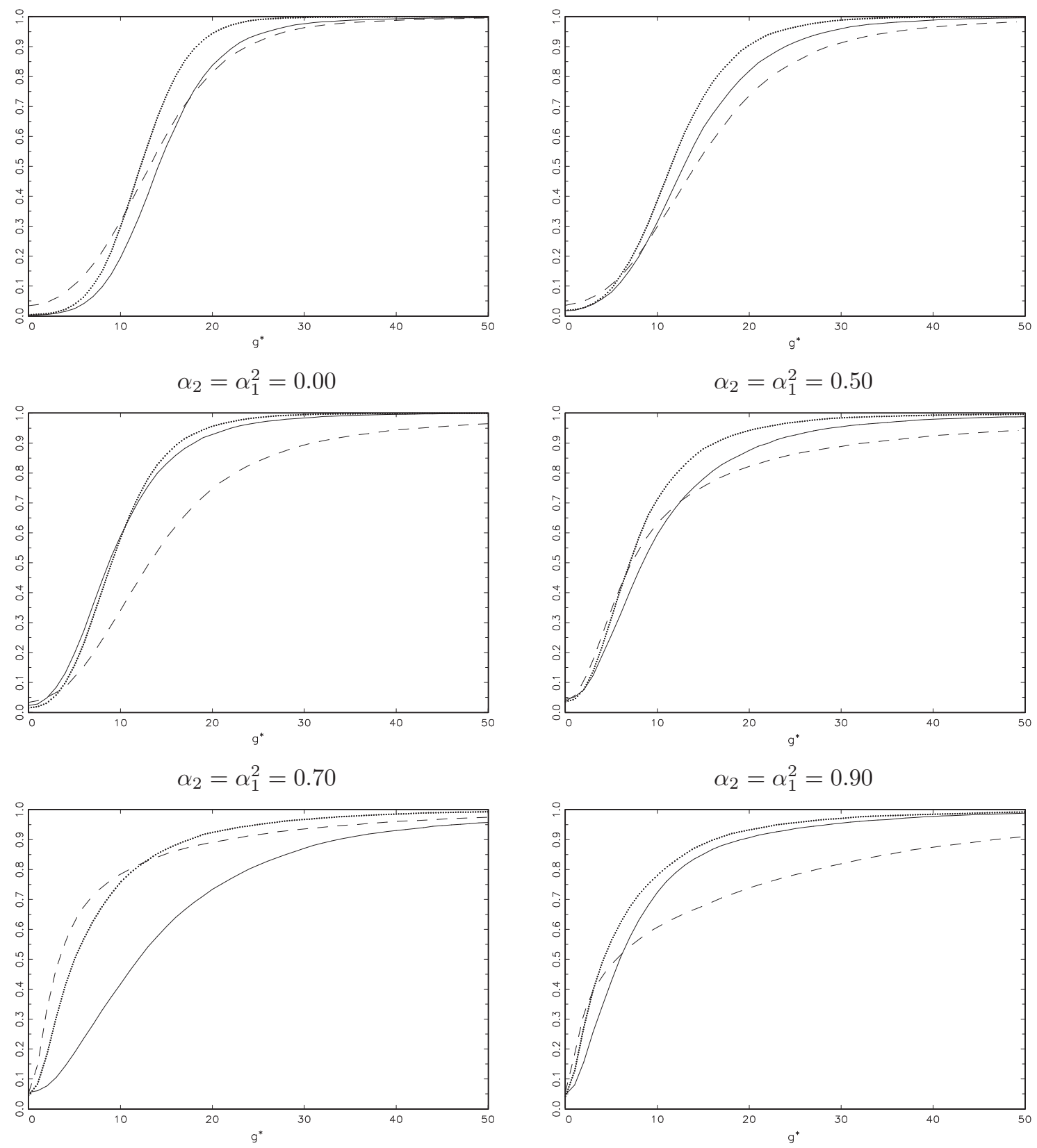

$\alpha_{2}=\alpha_{1}^{2}=0.95$

$\alpha_{2}=\alpha_{1}^{2}=1.00$

$W_{\omega_{1}, m, B V}(V R):---, W_{\omega_{2}, m, B V}(V R):$

$J S_{\delta_{m}}(V R)$

Figure S.6. Finite Sample Power, Seasonal Means, $\alpha_{0}=1$ 


\section{S.4.3. Individual Frequency Mean Shift Tests}

S.4.3.1. Empirical Size We begin by assessing the finite sample size properties of the seasonal mean shift tests, using data generated according to the following simulation DGP:

$$
\left(1-\alpha_{0} L\right)\left(1+\alpha_{1}^{2} L^{2}\right)\left(1+\alpha_{2} L\right) x_{t}=v_{t}, t=1, \ldots, T,
$$

where $v_{t} \sim \operatorname{NIID}(0,1)$ setting the initial conditions $x_{-3}=\cdots=x_{0}=0$. Results are, again, reported for a range of $\alpha_{i} \in\{1.00,0.95,0.9,0.7,0.5,0.0\}, i=0,2, \alpha_{1}^{2} \in$ $\{1.00,0.95,0.9,0.7,0.5,0.0\}$ and $T=\{100,240\}$.

Results relating to the Nyquist frequency mean shift tests are reported in Table S.12. Empirical size is seen to be closest to the nominal level for both tests when the data are near-integrated at the Nyquist frequency, with both tests again displaying some oversizing when the process if near-integrated at the Nyquist frequency and some undersizing when the process is stationary at the Nyquist frequency. The degree of undersizing is observed to be notably worse for the $S u p W_{\omega_{2}, m s, B V}(V R)$ test than for $S u p W_{\omega_{2}, m s, V}(V R)$ test.

Corresponding results for the annual frequency mean shift tests are reported in Table S.13. Empirical size results for these tests are broadly similar to the Nyquist frequency mean shift tests, with both tests displaying some oversizing when the data are nearintegrated at the annual frequency but exhibiting some undersize when the data are stationary at the annual frequency. The $S u p W_{\omega_{1}, m s, B V}(V R)$ is seen to display superior size control overall to the $S u p W_{\omega_{1}, m s, V}(V R)$ test, with the latter being quite severely oversized for $T=100$. While some modest oversize persists for the $\operatorname{Sup} W_{\omega_{1}, m s, B V}(V R)$ test when $T=240$, unreported simulations show that this diminishes for larger sample sizes, these being more reflective of the asymptotic critical values to which the test statistic is compared. 
Robust Tests for Deterministic Seasonality and Seasonal Mean Shifts

Table S.12. Finite Sample Size

Nyquist Frequency Mean Shift Tests

\begin{tabular}{|c|c|c|c|c|c|}
\hline & & \multicolumn{2}{|c|}{$T=100$} & \multicolumn{2}{|c|}{$T=240$} \\
\hline$\alpha_{2}$ & $\alpha_{0}=\alpha_{1}^{2}$ & $S u p W_{\omega_{2}, m s, V}(V R)$ & $S u p W_{\omega_{2}, m s, B V}(V R)$ & $S u p W_{\omega_{2}, m s, V}(V R)$ & $S u p W_{\omega_{2}, m s, B V}(V R)$ \\
\hline \multirow[t]{6}{*}{1.00} & 1.00 & 0.079 & 0.089 & 0.057 & 0.073 \\
\hline & 0.95 & 0.079 & 0.088 & 0.057 & 0.072 \\
\hline & 0.90 & 0.078 & 0.087 & 0.057 & 0.071 \\
\hline & 0.70 & 0.075 & 0.082 & 0.055 & 0.071 \\
\hline & 0.50 & 0.072 & 0.077 & 0.053 & 0.070 \\
\hline & 0.00 & 0.061 & 0.064 & 0.049 & 0.064 \\
\hline \multirow[t]{6}{*}{0.95} & 1.00 & 0.069 & 0.063 & 0.047 & 0.031 \\
\hline & 0.95 & 0.068 & 0.062 & 0.047 & 0.031 \\
\hline & 0.90 & 0.067 & 0.061 & 0.046 & 0.031 \\
\hline & 0.70 & 0.064 & 0.055 & 0.044 & 0.030 \\
\hline & 0.50 & 0.061 & 0.049 & 0.043 & 0.028 \\
\hline & 0.00 & 0.052 & 0.042 & 0.039 & 0.023 \\
\hline \multirow[t]{6}{*}{0.90} & 1.00 & 0.056 & 0.040 & 0.039 & 0.019 \\
\hline & 0.95 & 0.056 & 0.039 & 0.039 & 0.019 \\
\hline & 0.90 & 0.055 & 0.039 & 0.039 & 0.018 \\
\hline & 0.70 & 0.052 & 0.035 & 0.037 & 0.017 \\
\hline & 0.50 & 0.050 & 0.031 & 0.035 & 0.016 \\
\hline & 0.00 & 0.043 & 0.026 & 0.030 & 0.014 \\
\hline \multirow[t]{6}{*}{0.70} & 1.00 & 0.041 & 0.015 & 0.039 & 0.025 \\
\hline & 0.95 & 0.040 & 0.015 & 0.039 & 0.023 \\
\hline & 0.90 & 0.039 & 0.014 & 0.039 & 0.021 \\
\hline & 0.70 & 0.037 & 0.013 & 0.037 & 0.014 \\
\hline & 0.50 & 0.034 & 0.011 & 0.034 & 0.012 \\
\hline & 0.00 & 0.027 & 0.009 & 0.029 & 0.010 \\
\hline \multirow[t]{6}{*}{0.50} & 1.00 & 0.042 & 0.013 & 0.041 & 0.017 \\
\hline & 0.95 & 0.041 & 0.013 & 0.041 & 0.017 \\
\hline & 0.90 & 0.040 & 0.012 & 0.041 & 0.016 \\
\hline & 0.70 & 0.037 & 0.011 & 0.039 & 0.015 \\
\hline & 0.50 & 0.034 & 0.010 & 0.037 & 0.012 \\
\hline & 0.00 & 0.028 & 0.007 & 0.032 & 0.013 \\
\hline \multirow[t]{6}{*}{0.00} & 1.00 & 0.044 & 0.012 & 0.045 & 0.020 \\
\hline & 0.95 & 0.044 & 0.011 & 0.044 & 0.019 \\
\hline & 0.90 & 0.044 & 0.012 & 0.044 & 0.018 \\
\hline & 0.70 & 0.045 & 0.013 & 0.044 & 0.015 \\
\hline & 0.50 & 0.043 & 0.013 & 0.043 & 0.012 \\
\hline & 0.00 & 0.032 & 0.008 & 0.035 & 0.009 \\
\hline
\end{tabular}


Table S.13. Finite Sample Size

Annual Frequency Mean Shift Tests

\begin{tabular}{|c|c|c|c|c|c|}
\hline \multirow[b]{2}{*}{$\alpha_{1}^{2}$} & \multirow[b]{2}{*}{$\alpha_{0}=\alpha_{2}$} & \multicolumn{2}{|c|}{$T=100$} & \multicolumn{2}{|c|}{$\bar{T}=240$} \\
\hline & & $\operatorname{Sup} W_{\omega_{1}, m s, V}(V R)$ & $S u p W_{\omega_{1}, m s, B V}(V R)$ & $S u p W_{\omega_{1}, m s, V}(V R)$ & $S u p W_{\omega_{1}, m s, B V}(V R)$ \\
\hline \multirow[t]{6}{*}{1.00} & 1.00 & 0.091 & 0.079 & 0.053 & 0.053 \\
\hline & 0.95 & 0.091 & 0.079 & 0.053 & 0.053 \\
\hline & 0.90 & 0.089 & 0.079 & 0.053 & 0.053 \\
\hline & 0.70 & 0.084 & 0.077 & 0.051 & 0.053 \\
\hline & 0.50 & 0.080 & 0.072 & 0.048 & 0.051 \\
\hline & 0.00 & 0.074 & 0.068 & 0.046 & 0.048 \\
\hline \multirow[t]{6}{*}{0.95} & 1.00 & 0.101 & 0.086 & 0.066 & 0.070 \\
\hline & 0.95 & 0.100 & 0.087 & 0.065 & 0.069 \\
\hline & 0.90 & 0.099 & 0.087 & 0.065 & 0.068 \\
\hline & 0.70 & 0.094 & 0.084 & 0.062 & 0.063 \\
\hline & 0.50 & 0.089 & 0.079 & 0.058 & 0.059 \\
\hline & 0.00 & 0.082 & 0.073 & 0.055 & 0.053 \\
\hline \multirow[t]{6}{*}{0.90} & 1.00 & 0.102 & 0.080 & 0.057 & 0.074 \\
\hline & 0.95 & 0.101 & 0.080 & 0.057 & 0.072 \\
\hline & 0.90 & 0.099 & 0.080 & 0.056 & 0.070 \\
\hline & 0.70 & 0.093 & 0.076 & 0.054 & 0.061 \\
\hline & 0.50 & 0.088 & 0.070 & 0.052 & 0.050 \\
\hline & 0.00 & 0.082 & 0.065 & 0.049 & 0.041 \\
\hline \multirow[t]{6}{*}{0.70} & 1.00 & 0.068 & 0.053 & 0.048 & 0.061 \\
\hline & 0.95 & 0.067 & 0.052 & 0.048 & 0.060 \\
\hline & 0.90 & 0.066 & 0.053 & 0.047 & 0.058 \\
\hline & 0.70 & 0.063 & 0.050 & 0.044 & 0.052 \\
\hline & 0.50 & 0.059 & 0.044 & 0.042 & 0.047 \\
\hline & 0.00 & 0.056 & 0.036 & 0.040 & 0.044 \\
\hline \multirow[t]{6}{*}{0.50} & 1.00 & 0.055 & 0.035 & 0.049 & 0.036 \\
\hline & 0.95 & 0.054 & 0.035 & 0.048 & 0.035 \\
\hline & 0.90 & 0.053 & 0.036 & 0.047 & 0.033 \\
\hline & 0.70 & 0.050 & 0.037 & 0.045 & 0.029 \\
\hline & 0.50 & 0.048 & 0.039 & 0.043 & 0.027 \\
\hline & 0.00 & 0.045 & 0.038 & 0.040 & 0.027 \\
\hline \multirow[t]{6}{*}{0.00} & 1.00 & 0.043 & 0.004 & 0.044 & 0.010 \\
\hline & 0.95 & 0.043 & 0.005 & 0.044 & 0.010 \\
\hline & 0.90 & 0.042 & 0.005 & 0.044 & 0.010 \\
\hline & 0.70 & 0.041 & 0.010 & 0.044 & 0.008 \\
\hline & 0.50 & 0.041 & 0.015 & 0.042 & 0.008 \\
\hline & 0.00 & 0.042 & 0.021 & 0.041 & 0.010 \\
\hline
\end{tabular}


S.4.3.2. Empirical Power We next investigate the finite sample power properties of the individual frequency mean shift tests. To do so, data were generated according to the following simulation DGP:

$$
\begin{aligned}
x_{t} & =D U_{t, b 0} z_{t}^{\prime} \gamma_{b}+u_{t} \\
\left(1-\alpha_{0} L\right)\left(1+\alpha_{1}^{2} L^{2}\right)\left(1+\alpha_{2} L\right) u_{t} & =v_{t}, t=1, \ldots, T,
\end{aligned}
$$

where $v_{t} \sim \operatorname{NIID}(0,1)$ setting the initial conditions $u_{-3}=\cdots=u_{0}=0$. We set $T=240$ in order to present a fair comparison between the tests, given the severe oversize of the $\operatorname{Sup} W_{\omega_{1}, m s, V}(V R)$ test when $T=100$. We set $T_{b 0}:=0.5 T$ and $\gamma_{b}:=$ $\left(\gamma_{0, b}, \gamma_{1,1 b}, \gamma_{1,2 b}, \gamma_{2, b}\right)^{\prime}$.

Consider first the Nyquist frequency mean shift tests. Here we set $\gamma_{0, b}=\gamma_{1,1 b}=\gamma_{1,2 b}=$ 0 and examine the empirical rejection frequencies of the tests of $H_{0}: \gamma_{2, b}=0$ against $H_{1}: \gamma_{2, b} \neq 0$, at the nominal $5 \%$ level. We set $\alpha_{0}=\alpha_{1}^{2}=1$ and consider values of $\alpha_{2} \in\{0.00,0.50,0.70,0.90,0.95,1.00\}$ for a grid of 50 steps of values for $\gamma_{2, b}$ from to 0 to $\gamma_{2, b, \max }$ with the choices of $\gamma_{2, b, \max } \in\{0.20,0.50,1.00,6.00,8.00,15.00\}$ corresponding to the six choices of $\alpha_{2}$, respectively. The results are reported in Figure S.7. The best overall power performance is seen to be given by the $S u p W_{\omega_{2}, m s, B V}(V R)$ test, which displays almost uniformly greater power than the $\operatorname{Sup}_{\omega_{2}, m s, V}(V R)$ test when the data are near-integrated at the Nyquist frequency. While the $S u p W_{\omega_{2}, m s, B V}(V R)$ test has lower power than the $S u p W_{\omega_{2}, m s, V}(V R)$ test for low values of $\gamma_{2, b}$ when the data are stationary at the Nyquist frequency (due to the undersizing in the former noted above in these scenarios), the power of $S u p W_{\omega_{2}, m s, B V}(V R)$ quickly recovers as $\gamma_{2, b}$ is increased and is greater than the power of the $\operatorname{Sup} W_{\omega_{2}, m s, V}(V R)$ for larger values of $\gamma_{2, b}$.

We next report results relating to the finite sample power performance of the annual frequency mean shift tests. To do so we set $\gamma_{0, b}=\gamma_{2, b}=0$ and examine the empirical rejection frequencies of the tests of $H_{0}: \gamma_{1, b}=0$ against $H_{1}: \gamma_{1, b} \neq 0$ at the nominal $5 \%$ level, for a sample size of $T=240$, with $\alpha_{0}=\alpha_{2}=1$ and $\alpha_{1}^{2} \in\{0.00,0.50,0.70,0.90,0.95,1.00\}$. We set $\gamma_{1,1 b}=\gamma_{1,2 b}$ and consider a grid of 50 steps of values for $\gamma_{1,1 b}$ from to 0 to $\gamma_{1,1 b \text {,max }}$ with the choices of $\gamma_{1,1 b, \max } \in\{0.50,1.00,2.00,7.00,8.00,15.00\}$ corresponding to the four choices of $\alpha_{1}^{2}$, respectively. The results are reported in Figure S.8. Once again, no one test dominates across all scenarios considered, although the $\operatorname{Sup} W_{\omega_{1}, m s, B V}(V R)$ has the best overall power properties. The only scenario in which the $\operatorname{Sup} W_{\omega_{1}, m s, V}(V R)$ test displays significant power gains relative to the $\operatorname{Sup}_{\omega_{1}, m s, B V}(V R)$ test is when $\alpha_{1}^{2}=0.00$ or 0.5 and for lower values of $\gamma_{1,1 b}$; this can be attributed to the severe undersize of the $S u p W_{\omega_{1}, m s, B V}(V R)$ test in this scenario although, once again, the power of this test recovers for larger break magnitudes and is greater than the power of the $S u p W_{\omega_{1}, m s, V}(V R)$ test for larger values of $\gamma_{1, b}$. These finite sample results are consistent with the asymptotic local power results reported in Figure S.3, where it can be see that the $S u p W_{\omega_{1}, m s, B V}(V R)$ has far greater power than the $S u p W_{\omega_{1}, m s, V}(V R)$ test when the data is near-integrated at the annual frequency, with the undersize the $\operatorname{Sup} W_{\omega_{1}, m s, B V}(V R)$ exhibits as $\overline{\alpha_{1}}$ increases having a detrimental impact on the power of the test for breaks of small magnitude in more stationary scenarios. 

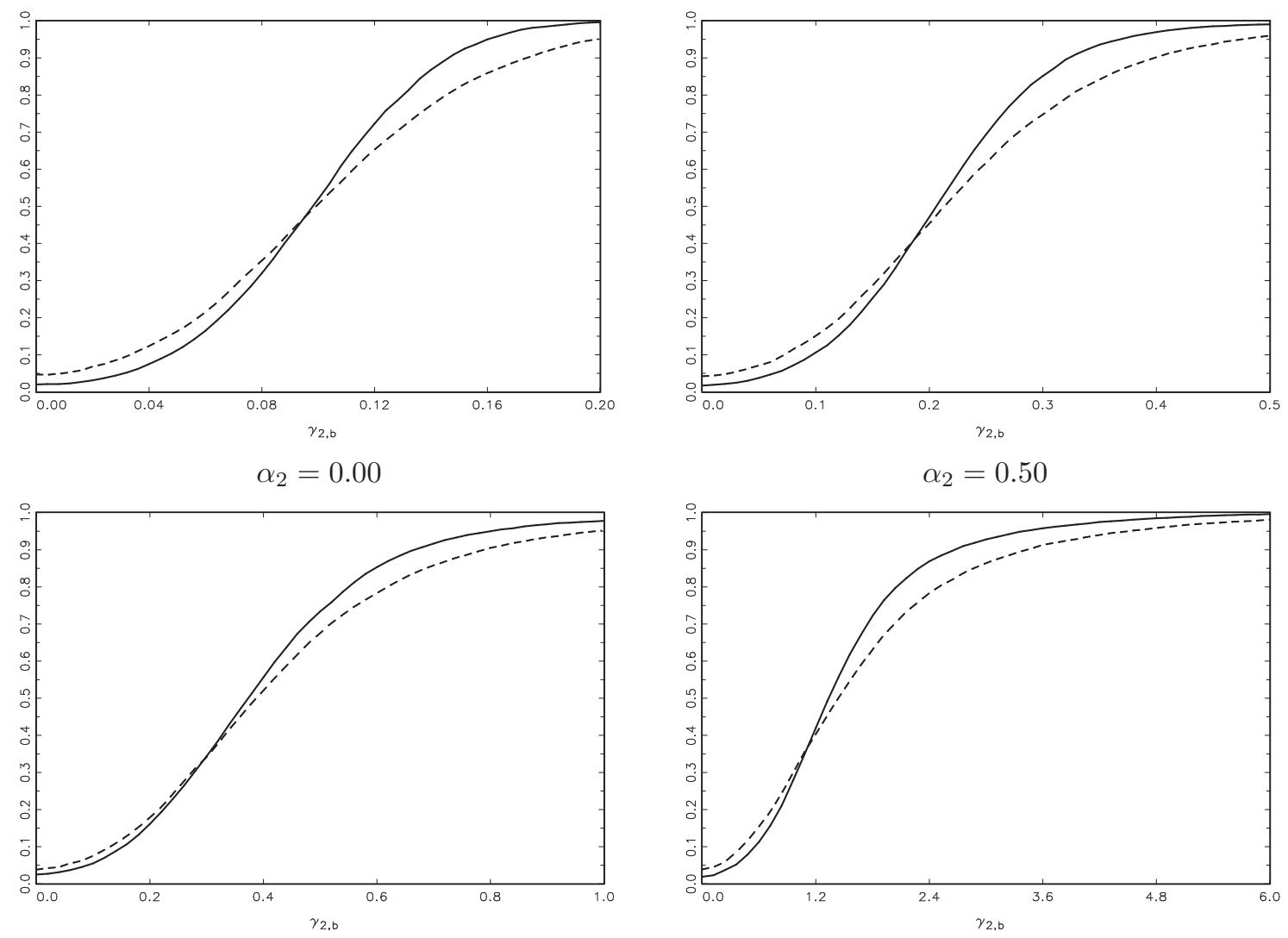

$\alpha_{2}=0.70$

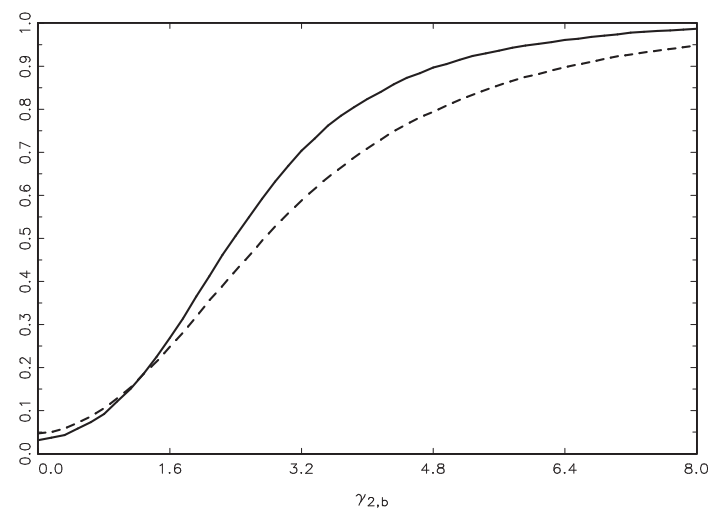

$\alpha_{2}=0.90$

$$
\alpha_{2}=0.95
$$

$\operatorname{Sup} W_{\omega_{2}, m s, V}(V R):-\cdots-$ - $S u p W_{\omega_{2}, m s, B V}(V R)$ :

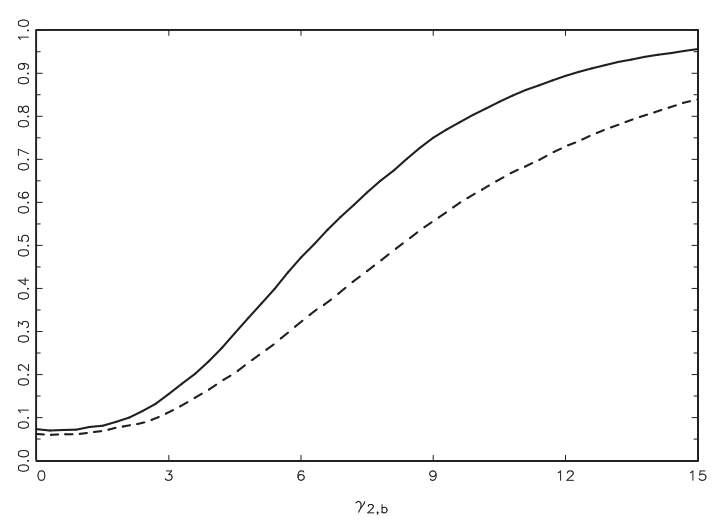

$\alpha_{2}=1.00$

Figure S.7. Finite Sample Power, Nyquist Frequency Mean Shift Tests, $\alpha_{0}=\alpha_{1}^{2}=1$ 

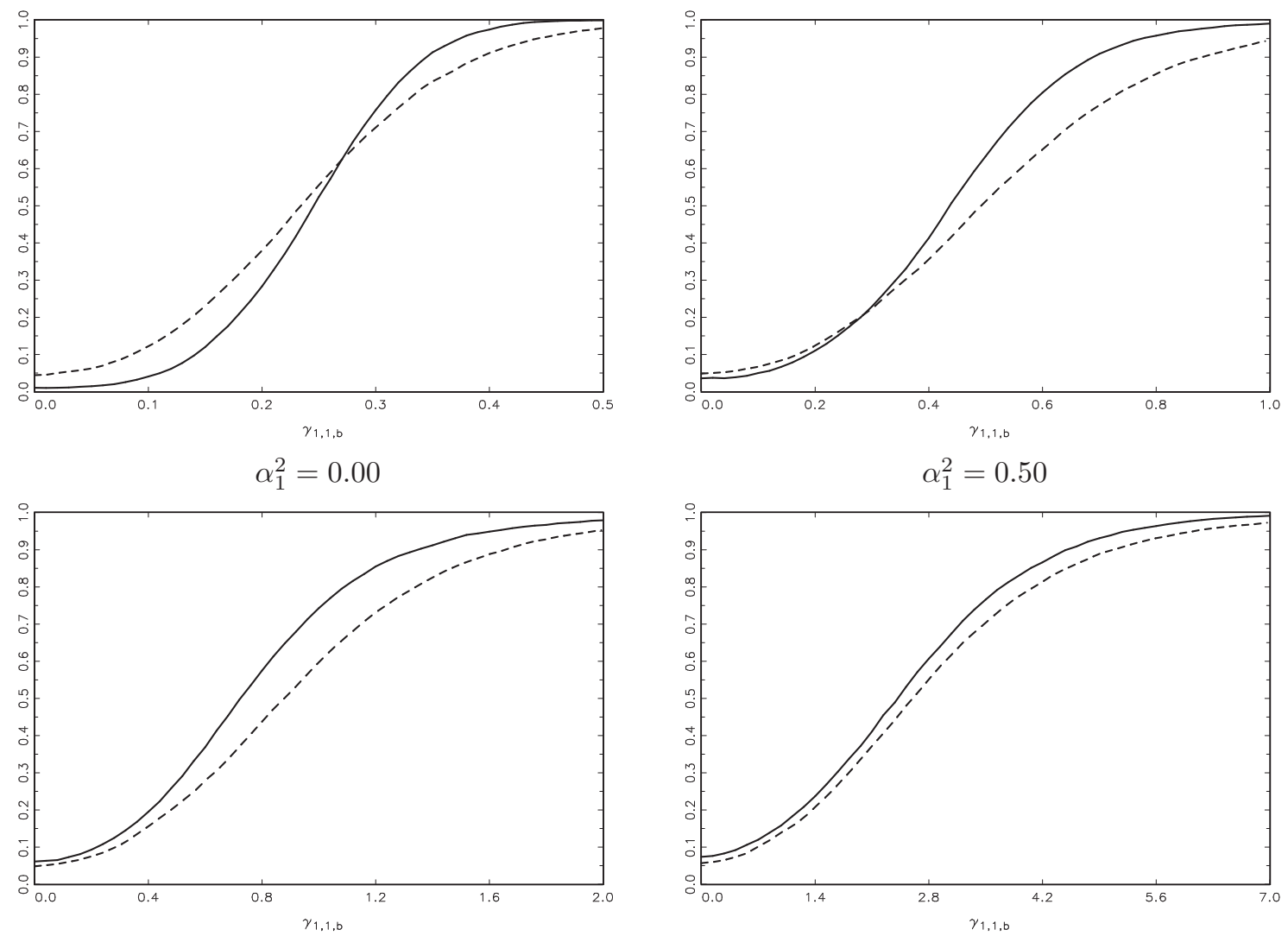

$\alpha_{1}^{2}=0.70$

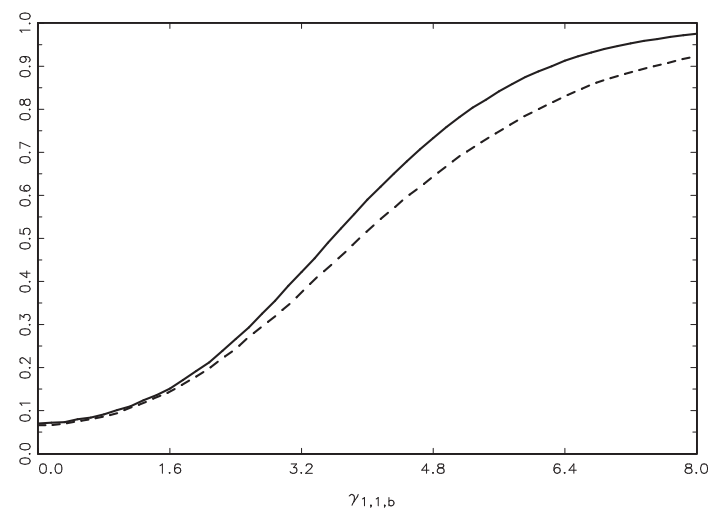

$\alpha_{1}^{2}=0.90$

$$
\alpha_{1}^{2}=0.95
$$

$\operatorname{Sup} W_{\omega_{1}, m s, V}(V R):-\cdots-\operatorname{Sup}_{\omega_{1}, m s, B V}(V R)$ :

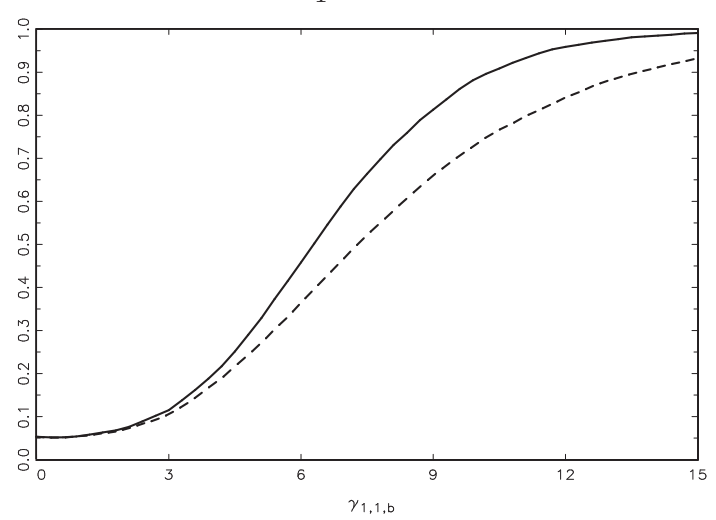

$\alpha_{1}^{2}=1.00$

Figure S.8. Finite Sample Power, Annual Frequency Mean Shift Tests, $\alpha_{0}=\alpha_{2}=1$ 


\section{S.4.4. Joint Frequency Seasonal Mean Shift Tests}

We next examine the finite sample size and power properties of the $J S_{\delta_{m s}}(V R)$ test.

S.4.4.1. Empirical Size We begin by examining the size performance of the jointfrequency $J S_{\delta_{m s}}(V R)$ test under the null of no shifts in deterministic seasonality. To that end, data were simulated according to the following simulation DGP:

$$
\left(1-\alpha_{0} L\right)\left(1+\alpha_{1}^{2} L^{2}\right)\left(1+\alpha_{2} L\right) x_{t}=v_{t}, t=1, \ldots, T,
$$

with $v_{t} \sim N I I D(0,1)$ setting the initial conditions $x_{-3}=\cdots=x_{0}=0$. The $J S_{\delta_{m s}}(V R)$ test was performed at the nominal asymptotic $5 \%$ level and results are reported in Table S.14 for a range of $\alpha_{2} \in\{1.00,0.95,0.9,0.7,0.5,0.0\}, \alpha_{1}^{2} \in\{1.00,0.95,0.9,0.7,0.5,0.0\}$ setting $\alpha_{0}=1$. Results are reported for sample sizes of $T=\{100,240\}$. The results suggest that the proposed test procedure has decent overall finite sample size control, although it exhibits some oversizing when the data are near-integrated as both seasonal frequencies and some undersizing when the data are stationary at both seasonal frequencies and some oversizing when the data are near-integrated at both seasonal frequencies. In each case this is as a consequence of the size distortions exhibited by the tests used in its construction in these scenarios discussed above. For example, the undersize exhibited by the $J S_{\delta_{m s}}(V R)$ test when the data are stationary at both frequencies mirrors the undersize exhibited by the $\operatorname{Sup} W_{\omega_{i}, m s, B V}(V R)$ tests when the data are stationary at frequency $\omega_{i}, i=1,2$.

Table S.14. Finite Sample Size $J S_{\delta_{m s}}(V R)$ Test $\alpha_{0}=1$

\begin{tabular}{rcccccc}
\multicolumn{7}{c}{$T=100$} \\
\hline$\alpha_{2}$ & 1.00 & 0.95 & 0.90 & 0.70 & 0.50 & 0.00 \\
\hline 1.00 & 0.100 & 0.089 & 0.081 & 0.074 & 0.072 & 0.065 \\
0.95 & 0.101 & 0.088 & 0.081 & 0.077 & 0.074 & 0.069 \\
0.90 & 0.091 & 0.079 & 0.073 & 0.068 & 0.065 & 0.062 \\
0.70 & 0.060 & 0.049 & 0.042 & 0.038 & 0.035 & 0.033 \\
0.50 & 0.046 & 0.031 & 0.024 & 0.020 & 0.020 & 0.015 \\
0.00 & 0.023 & 0.014 & 0.006 & 0.002 & 0.001 & 0.001 \\
\hline
\end{tabular}

\begin{tabular}{rcccccc}
\multicolumn{7}{c}{$T=240$} \\
\hline$\alpha_{2}$ & 1.00 & 0.95 & 0.90 & 0.70 & 0.50 & 0.00 \\
\hline 1.00 & 0.063 & 0.053 & 0.051 & 0.047 & 0.046 & 0.047 \\
0.95 & 0.075 & 0.065 & 0.062 & 0.055 & 0.054 & 0.059 \\
0.90 & 0.071 & 0.060 & 0.058 & 0.049 & 0.047 & 0.054 \\
0.70 & 0.052 & 0.033 & 0.030 & 0.029 & 0.029 & 0.028 \\
0.50 & 0.037 & 0.016 & 0.014 & 0.012 & 0.013 & 0.008 \\
0.00 & 0.024 & 0.006 & 0.003 & 0.002 & 0.001 & 0.000 \\
\hline
\end{tabular}

S.4.4.2. Empirical Power Finally, we examine the finite sample power of the $J S_{\delta_{m s}}(V R)$ test to detect shifts in deterministic seasonality under the alternative that both a Nyquist 
and annual frequency mean shift are present in the data. We expect the rejection rate of the $J S_{\delta_{m s}}(V R)$ test to be greater than either of the individual $S u p W_{\omega_{1}, m s, B V}(V R)$ and $\operatorname{Sup}_{\omega_{2}, m s, B V}(V R)$ tests in this setting.

To this end, data were generated according to the following simulation DGP:

$$
\begin{aligned}
x_{t} & =D U_{t, b 0} z_{t}^{\prime} \gamma_{b}+u_{t} \\
\left(1-\alpha_{0} L\right)\left(1+\alpha_{1}^{2} L^{2}\right)\left(1+\alpha_{2} L\right) u_{t} & =v_{t}, t=1, \ldots, T,
\end{aligned}
$$

where $v_{t} \sim \operatorname{NIID}(0,1)$ setting the initial conditions $u_{-3}=\cdots=u_{0}=0$ We set $\gamma_{b}=\left(0, \lambda_{A}^{-1} g^{*}, \lambda_{A}^{-1} g *, \lambda_{N}^{-1} g^{*}\right)^{\prime}$ for a range of values of $g^{*}$ from 1 to 50 Figure S.9 graphs power curves for the $J S_{\delta_{m s}}(V R), \operatorname{Sup} W_{\omega_{1}, m s, B V}(V R)$ and $S u p W_{\omega_{2}, m s, B V}(V R)$ tests for $\alpha_{0}=1.00$ and $\alpha_{1}^{2}=\alpha_{2}=\{0.00,0.50,0.70,0.90,0.95,1.00\}$ with the values $\lambda_{A}=\{0.75,1.00,2.00,7.00,8.00,15.00\}$ and $\lambda_{N}=\{0.50,0.50,1.00,6.00,8.00,15.00\}$ corresponding to the six scenarios considered. The values of $\lambda_{A}$ and $\lambda_{N}$ are, again, chosen such that the grid of alternatives at each frequency closely mirrors those used for when examining each individual frequency test. It is seen from these power curves that across a majority of scenarios the $J S_{\delta_{m s}}(V R)$ test has greater overall power than either of the constituent tests used in its construction. The one exception is when $\alpha_{1}^{2}=\alpha_{2}=0.00$, with the severe undersize exhibited by the $S u p W_{\omega_{1}, m s, B V}(V R)$ test correspondingly negatively impacting on the power of the $J S_{\delta_{m s}}(V R)$ test. The power of the $J S_{\delta_{m s}}(V R)$ test recovers fairly rapidly as the magnitude of the breaks increase, however. 

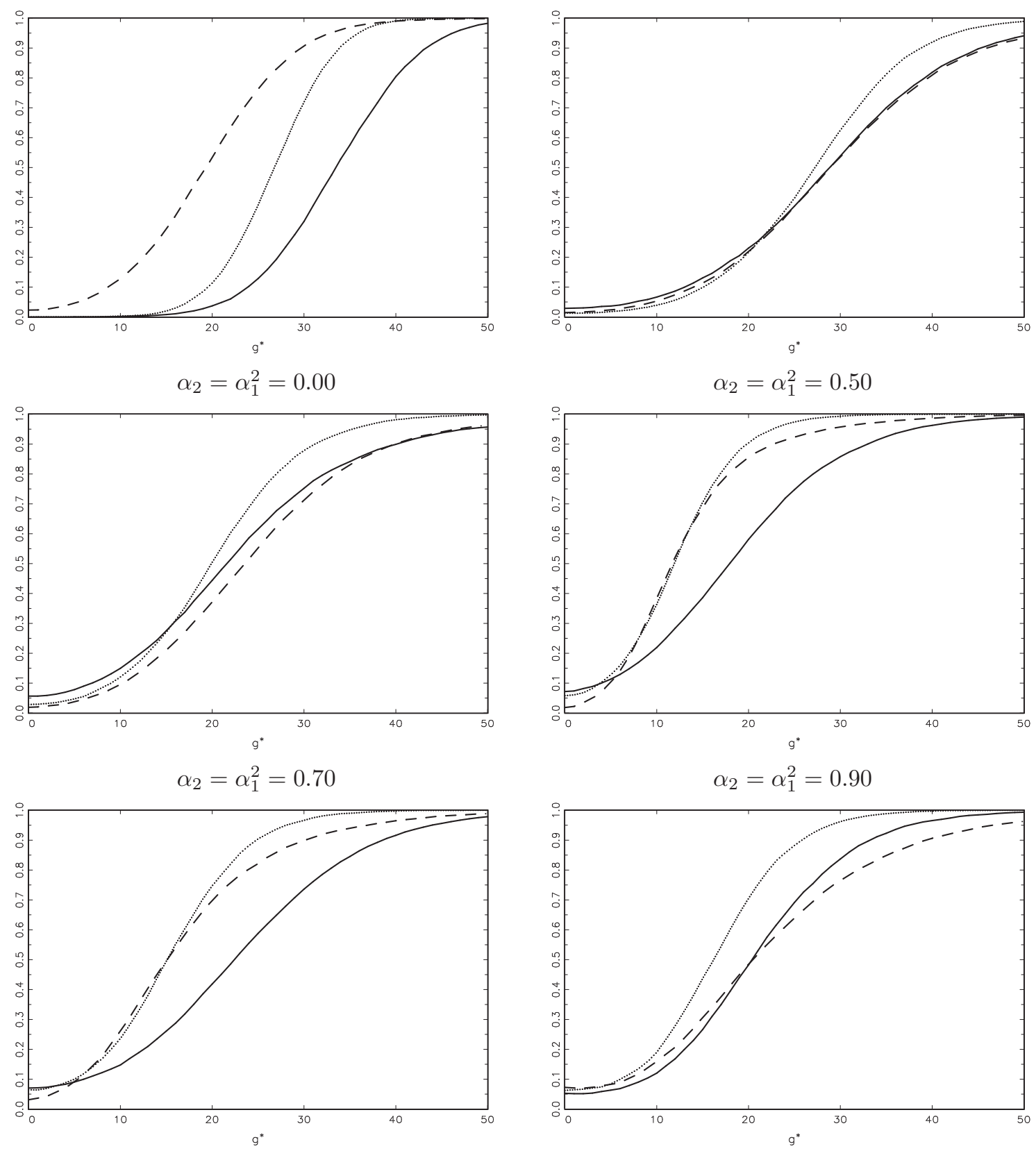

$\alpha_{2}=\alpha_{1}^{2}=0.95$

$$
\alpha_{2}=\alpha_{1}^{2}=1.00
$$

$\operatorname{Sup} W_{\omega_{2}, m s, B V}(V R):---, \operatorname{Sup} W_{\omega_{1}, m s, B V}(V R):-, J S_{\delta_{m s}}(V R)$ :

Figure S.9. Finite Sample Power, Seasonal Mean Shift, $\alpha_{0}=1$ 


\section{S.5. PROOF OF THEOREMS S.2 - S.5}

In order to prove the limiting distributions of the test statistics we will need to make use of the following lemmas.

Lemma S.2. Let the conditions of Theorem S.2 hold. Then as $T \rightarrow \infty$ if the data are $I_{\omega_{i}}(0)$,

and if the data are $I_{\omega_{i}}(1)$

$$
T^{1 / 2} \tilde{\delta}_{\omega_{i}, m} \stackrel{d}{\rightarrow} \Omega_{i, 0}^{1 / 2}\left[P_{i, 0}^{\delta_{m}}+\Psi_{i}^{\delta_{m}} \kappa_{\omega_{i}, m}\right],
$$

$$
T^{-1 / 2} \tilde{\delta}_{\omega_{i}, m} \stackrel{d}{\rightarrow} \Omega_{i, 1}^{1 / 2}\left[P_{i, 1}^{\delta_{m}}+\Psi_{i}^{\delta_{m}} \kappa_{\omega_{i}, m}\right] .
$$

Proof. The OLS estimate of $\delta_{\omega_{i}, m}$ under the the local alternative given in Definition 4.1 is given by

$$
\begin{aligned}
\tilde{\delta}_{\omega_{i}, m} & =\left[\sum_{t=1}^{T} F_{i, m}(t) F_{i, m}(t)^{\prime}\right]^{-1}\left[\sum_{t=1}^{T} F_{i, m}(t)\right] \delta_{\omega_{i}, m} \\
& +\left[\sum_{t=1}^{T} F_{i, m}(t) F_{i, m}(t)^{\prime}\right]^{-1} \sum_{t=1}^{T} F_{i, m}(t) u_{i, t} .
\end{aligned}
$$

When $u_{i, t}$ is $I_{\omega_{i}}(0)$ we have

$$
\begin{aligned}
T^{1 / 2} \tilde{\delta}_{\omega_{i}, m} & =\left[T^{-1} \sum_{t=1}^{T} F_{i, m}(t) F_{i, m}(t)^{\prime}\right]^{-1}\left[T^{-1} \sum_{t=1}^{T} F_{i, m}(t)\right] T^{1 / 2} \delta_{\omega_{i}, m} \\
& +\left[T^{-1} \sum_{t=1}^{T} F_{i, m}(t) F_{i, m}(t)^{\prime}\right]^{-1} T^{-1 / 2} \sum_{t=1}^{T} F_{i, m}(t) u_{i, t} .
\end{aligned}
$$

The first term is given by

$$
\left[T^{-1} \sum_{t=1}^{T} F_{i, m}(t) F_{i, m}(t)^{\prime}\right]^{-1}\left[T^{-1} \sum_{t=1}^{T} F_{i, m}(t)\right] T^{1 / 2} \delta_{\omega_{i}, m} \stackrel{d}{\rightarrow} \Omega_{i, 0}^{1 / 2} \Psi_{i}^{\delta_{m}} \kappa_{\omega_{i}, m},
$$

and the second term is given by

$$
\left[T^{-1} \sum_{t=1}^{T} F_{i, m}(t) F_{i, m}(t)^{\prime}\right]^{-1} T^{-1 / 2} \sum_{t=1}^{T} F_{i, m}(t) u_{i, t} .
$$

From Lemma S.1 we have that

$$
\begin{aligned}
{\left[T^{-1} \sum_{t=1}^{T} F_{i, m}(t) F_{i, m}(t)^{\prime}\right]^{-1} T^{-1 / 2} \sum_{t=1}^{T} F_{i, m}(t) u_{i, t} \stackrel{d}{\rightarrow}\left[\int_{0}^{1} F_{i, m}(r) F_{i, m}(r)^{\prime} d r\right]^{-1} B_{i, 0}^{\delta_{m}} } \\
=: \Omega_{i, 0}^{1 / 2} P_{i, 0}^{\delta_{m}}
\end{aligned}
$$

so that

$$
T^{1 / 2} \tilde{\delta}_{\omega_{i}, m} \stackrel{d}{\rightarrow} \Omega_{i, 0}^{1 / 2}\left[P_{i, 0}^{\delta_{m}}+\Psi_{i}^{\delta_{m}} \kappa_{\omega_{i}, m}\right] .
$$

When the data are $I_{\omega_{i}}(1)$ we have that 


$$
\begin{aligned}
T^{-1 / 2} \tilde{\delta}_{\omega_{i}, m} & =\left[T^{-1} \sum_{t=1}^{T} F_{i, m}(t) F_{i, m}(t)^{\prime}\right]^{-1}\left[T^{-1} \sum_{t=1}^{T} F_{i, m}(t)\right] T^{-1 / 2} \delta_{\omega_{i}, m} \\
& +\left[T^{-1} \sum_{t=1}^{T} F_{i, m}(t) F_{i, m}(t)^{\prime}\right]^{-1} T^{-1} \sum_{t=1}^{T} T^{-1 / 2} F_{i, m}(t) u_{i, t} .
\end{aligned}
$$

The first term is given by

$$
\left[T^{-1} \sum_{t=1}^{T} F_{i, m}(t) F_{i, m}(t)^{\prime}\right]^{-1}\left[T^{-1} \sum_{t=1}^{T} F_{i, m}(t)\right] T^{-1 / 2} \delta_{\omega_{i}, m} \stackrel{d}{\rightarrow} \Omega_{i, 1}^{1 / 2} \Psi_{i}^{\delta_{m}} \kappa_{\omega_{i}, m},
$$

and the second term is given by

$$
\left[T^{-1} \sum_{t=1}^{T} F_{i, m}(t) F_{i, m}(t)^{\prime}\right]^{-1} T^{-1} \sum_{t=1}^{T} T^{-1 / 2} F_{i, m}(t) u_{i, t} .
$$

Using the result from Lemma S.1 we, thus, have that

$$
\begin{aligned}
{\left[T^{-1} \sum_{t=1}^{T} F_{i, m}(t) F_{i, m}(t)^{\prime}\right]^{-1} T^{-1} \sum_{t=1}^{T} T^{-1 / 2} F_{i, m}(t) u_{i, t} \stackrel{d}{\rightarrow}\left[\int_{0}^{1} F_{i, m}(r) F_{i, m}(r)^{\prime} d r\right]^{-1} B_{i, 1}^{\delta_{m}} } \\
=: \Omega_{i, 1}^{1 / 2} P_{i, 1}^{\delta_{m}},
\end{aligned}
$$

so that

$$
T^{-1 / 2} \tilde{\delta}_{\omega_{i}, m} \stackrel{d}{\rightarrow} \Omega_{i, 1}^{1 / 2}\left[P_{i, 1}^{\delta_{m}}+\Psi_{i}^{\delta_{m}} \kappa_{\omega_{i}, m}\right]
$$

Lemma S.3. Let the conditions of Theorem S.2 hold. Defining $\tilde{S}_{i, t}=\sum_{s=1}^{t} z_{i, s} \tilde{u}_{i, s}$, then:

(a) if the data are $I_{\omega_{i}}(0)$ then as $T \rightarrow \infty$

(i)

(ii)

$$
T^{-1 / 2} \tilde{S}_{i,\lfloor r T\rfloor} \stackrel{d}{\rightarrow} \Omega_{i, 0}^{1 / 2}\left[W^{i}(r)-\left[\int_{0}^{r} \breve{F}_{i, m}(s) d s\right] P_{i, 0}^{\delta_{m}}\right]=: \Omega_{i, 0}^{1 / 2} Q_{i, 0}^{\delta_{m}}(r)
$$

$$
\begin{gathered}
\tilde{\sigma}_{V, \omega_{i}}^{2} \stackrel{d}{\rightarrow} \Omega_{i, 0} \int_{0}^{1} Q_{i, 0}^{\delta_{m}}(r) Q_{i, 0}^{\delta_{m}}(r)^{\prime} d r \\
\tilde{\sigma}_{B V, \omega_{i}}^{2} \stackrel{d}{\rightarrow} \Omega_{i, 0} \Phi_{i, 0}\left(b, k^{*}(\cdot)\right)
\end{gathered}
$$

(b) if the data are $I_{\omega_{i}}(1)$ then as $T \rightarrow \infty$

(i)

$$
T^{-3 / 2} \tilde{S}_{i,\lfloor r T\rfloor} \stackrel{d}{\rightarrow} \Omega_{i, 1}^{1 / 2}\left[\int_{0}^{r} V^{i}(s) d s-\left[\int_{0}^{r} \breve{F}_{i, m}(s) d s\right] P_{i, 1}^{\delta_{m}}\right]=: \Omega_{i, 1}^{1 / 2} Q_{i, 1}^{\delta_{m}}(r, \lambda)
$$

(ii)

$$
\begin{aligned}
T^{-2} \tilde{\sigma}_{V, \omega_{i}}^{2} \stackrel{d}{\rightarrow} \Omega_{i, 1} \int_{0}^{1} Q_{i, 1}^{\delta_{m}}(r) Q_{i, 1}^{\delta_{m}}(r)^{\prime} d r \\
T^{-2} \tilde{\sigma}_{B V, \omega_{i}}^{2} \stackrel{d}{\rightarrow} \Omega_{i, 1} \Phi_{i, 1}\left(b, k^{*}(\cdot)\right)
\end{aligned}
$$


Proof. We can write the residuals from the OLS estimation of (3.5) under both the null and alternative hypothesis as $\tilde{u}_{i, t}=u_{i, t}-F_{i, m}(t)^{\prime} \tilde{\delta}_{\omega_{i}, m}$ so that $z_{i, t} \tilde{u}_{i, t}=z_{i, t} u_{i, t}-$ $\breve{F}_{i, m}(t)^{\prime} \tilde{\delta}_{\omega_{i}, m}$. Summing yields

$$
\tilde{S}_{i,\lfloor r T\rfloor}=\sum_{t=1}^{\lfloor r T\rfloor} z_{i, t} u_{i, t}-\sum_{t=1}^{\lfloor r T\rfloor} \breve{F}_{i, m}(t)^{\prime} \tilde{\delta}_{\omega_{i}, m} .
$$

When the date are $I_{\omega_{i}}(0)$ we have

$$
\begin{aligned}
T^{-1 / 2} \tilde{S}_{i,\lfloor r T\rfloor} & =T^{-1 / 2} \sum_{t=1}^{\lfloor r T\rfloor} z_{i, t} u_{i, t}-T^{-1} \sum_{t=1}^{\lfloor r T\rfloor} \breve{F}_{i, m}(t)^{\prime} T^{1 / 2} \tilde{\delta}_{\omega_{i}, m} \\
& \stackrel{d}{\rightarrow} \Omega_{i, 0}^{1 / 2}\left[W^{i}(r)-\left[\int_{0}^{r} \breve{F}_{i, m}(s)^{\prime} d s\right] P_{i, 0}^{\delta_{m}}\right]
\end{aligned}
$$

from Lemma S.1 and Lemma S.2 and the CMT, proving part (a)(i) of this Lemma. The proof of (a)(ii) follows directly from part (a)(i), the CMT and Theorem 1 of Bunzel and Vogelsang (2005).

When the date are $I_{\omega_{i}}(1)$ we have

$$
\begin{aligned}
T^{3 / 2} \tilde{S}_{i,\lfloor r T\rfloor} & =T^{-1} \sum_{t=1}^{\lfloor r T\rfloor} T^{-1 / 2} z_{i, t} u_{i, t}-T^{-1} \sum_{t=1}^{\lfloor r T\rfloor} \breve{F}_{i, m}(t)^{\prime} T^{-1 / 2} \tilde{\delta}_{\omega_{i}, m} \\
& \stackrel{d}{\rightarrow} \Omega_{i, 1}^{1 / 2}\left[\int_{0}^{r} V^{i}(r)-\left[\int_{0}^{r} \breve{F}_{i, m}(s)^{\prime} d s\right] P_{i, 1}^{\delta}\right],
\end{aligned}
$$

from Lemma S.1 and Lemma S.2 and the CMT, proving part (b)(i) of this Lemma. The proof of (b)(ii) follows directly from part (b)(i), the CMT and Theorem 1 of Bunzel and Vogelsang (2005).

\section{Proof of Theorem S.2:}

(a) When the data are $I_{\omega_{1}}(0)$ we have that

$$
\begin{aligned}
W_{\omega_{1}, m, V} & =\left(T^{1 / 2} \tilde{\delta}_{\omega_{1}, m}\right)^{\prime}\left[\tilde{\sigma}_{V, \omega_{1}}^{2}\left[T^{-1} \sum_{t=1}^{T} F_{1, m}(t) F_{1, m}(t)^{\prime}\right]^{-1}\right]^{-1}\left(T^{1 / 2} \tilde{\delta}_{\omega_{i}, m}\right) \\
& \stackrel{d}{\rightarrow}\left[P_{1,0}^{\delta_{m}}+\Psi_{1}^{\delta_{m}} \kappa_{\omega_{1}, m}\right]^{\prime}\left[\int_{0}^{1} Q_{1,1}^{\delta_{m}}(r) Q_{1,1}^{\delta_{m}}(r)^{\prime} d r\left[\int_{0}^{1} F_{1, m}(r) F_{1, m}(r)^{\prime} d r\right]^{-1}\right]^{-1} \\
& \times\left[P_{1,0}^{\delta_{m}}+\Psi_{1}^{\delta_{m}} \kappa_{\omega_{1}, m}\right]
\end{aligned}
$$

by Lemmas S.2 and S.3 and the CMT. Moreover,

$$
\begin{aligned}
W_{\omega_{1}, m, B V} & =\left(T^{1 / 2} \tilde{\delta}_{\omega_{1}, m}\right)^{\prime}\left[\tilde{\sigma}_{B V, \omega_{1}}^{2}\left[T^{-1} \sum_{t=1}^{T} F_{1, m}(t) F_{1, m}(t)^{\prime}\right]^{-1}\right]^{-1}\left(T^{1 / 2} \tilde{\delta}_{\omega_{1}, m}\right) \\
& \stackrel{d}{\rightarrow}\left[P_{1,0}^{\delta_{m}}+\Psi_{1}^{\delta_{m}} \kappa_{\omega_{1}, m}\right]^{\prime}\left[\Phi_{1,0}\left(b, k^{*}(\cdot)\right)\left[\int_{0}^{1} F_{1, m}(r) F_{1, m}(r)^{\prime} d r\right]^{-1}\right]^{-1} \\
& \times\left[P_{1,0}^{\delta_{m}}+\Psi_{1}^{\delta_{m}} \kappa_{\omega_{1}, m}\right]
\end{aligned}
$$


by Lemmas S.2 and S.3 and the CMT.

The proof of part (b) of this theorem follows from part (a) and the CMT.

\section{Proof of Theorem S.3:}

(a) If the data are $I_{\omega_{1}}(1)$

$$
\begin{aligned}
W_{\omega_{1}, m, V} & =\left(T^{-1 / 2} \tilde{\delta}_{\omega_{1}, m}\right)^{\prime}\left[T^{-2} \tilde{\sigma}_{V, \omega_{1}}^{2}\left[T^{-1} \sum_{t=1}^{T} F_{1, m}(t) F_{1, m}(t)^{\prime}\right]^{-1}\left(T^{-1 / 2} \tilde{\delta}_{\omega_{1}, m}\right)\right. \\
& \stackrel{d}{\rightarrow}\left[P_{1,1}^{\delta_{m}}+\Psi_{1}^{\delta_{m}} \kappa_{\omega_{1}, m}\right]^{\prime}\left[\int_{0}^{1} Q_{1,1}^{\delta_{m}}(r)^{\prime} Q_{1,1}^{\delta_{m}}(r) d r\left[\int_{0}^{1} F_{1, m}(r) F_{1, m}(r)^{\prime} d r\right]^{-1}\right]^{-1} \\
& \times\left[P_{1,1}^{\delta_{m}}+\Psi_{1}^{\delta_{m}} \kappa_{\omega_{1}, m}\right]
\end{aligned}
$$

by Lemmas S.2 and S.3 and the CMT. Moreover,

$$
\begin{aligned}
W_{\omega_{1}, m, B V} & =\left(T^{-1 / 2} \tilde{\delta}_{\omega_{1}, m}\right)^{\prime}\left[T^{-2} \tilde{\sigma}_{B V, \omega_{1}}^{2}\left[T^{-1} \sum_{t=1}^{T} F_{1, m}(t) F_{1, m}(t)^{\prime}\right]^{-1}\right]^{-1}\left(T^{-1 / 2} \tilde{\delta}_{\omega_{1}, m}\right) \\
& \stackrel{d}{\rightarrow}\left[P_{1,1}^{\delta_{m}}+\Psi_{1}^{\delta_{m}} \kappa_{\omega_{1}, m}\right]^{\prime}\left[\Phi_{1,1}\left(b, k^{*}(\cdot)\right)\left[\int_{0}^{1} F_{1, m}(r) F_{1, m}(r)^{\prime} d r\right]^{-1}\right]^{-1} \\
& \times\left[P_{1,1}^{\delta_{m}}+\Psi_{1}^{\delta_{m}} \kappa_{\omega_{1}, m}\right]
\end{aligned}
$$

by Lemmas S.2 and S.3 and the CMT.

The proof of part (b) of this theorem follows from part (a) and the CMT.

The proof of Theorems S.4 and S.5 follow in a similar manner to the proofs of Theorems S.2 and S.3, respectively.

\section{S.6. PROOF OF THEOREMS S.6 - S.10}

In order to prove the limiting distributions of the test statistics we will need to make use of the following lemmas.

LEMmA S.4. Let the filtered series $x_{i, t}$ be generated according to (3.4) and let Assumption 2.1 hold. Then as $T \rightarrow \infty$, if the data are $I_{\omega_{i}}(0)$

$$
T^{-1 / 2} \sum_{t=1}^{T} F_{i}(t, \lambda) u_{i, t} \stackrel{d}{\rightarrow}\left[\begin{array}{c}
\Omega_{i, 0}^{1 / 2} B_{i, 0}^{\delta_{m}} \\
\Omega_{i, 0}^{1 / 2} B_{i, 0}^{\delta_{m s}}
\end{array}\right]=: B_{i, 0}
$$

where

$$
\begin{aligned}
& B_{1,0}^{\delta_{m}}=\left[W_{1}(1), W_{3}(1)\right]^{\prime}, B_{2,0}^{\delta_{m}}=\left[W_{2}(1)\right], \\
& B_{1,0}^{\delta_{m s}}=\left[W_{1}(1)-W_{1}(\lambda), W_{3}(1)-W_{3}(\lambda)\right]^{\prime}, B_{2,0}^{\delta_{m s}}=\left[W_{2}(1)-W_{2}(\lambda)\right],
\end{aligned}
$$

while if the data are $I_{\omega_{i}}(1)$ then

$$
T^{-3 / 2} \sum_{t=1}^{T} F_{i}(t, \lambda) u_{i, t} \stackrel{d}{\rightarrow}\left[\begin{array}{c}
\Omega_{i, 1}^{1 / 2} B_{i, 1}^{\delta_{m}} \\
\Omega_{i, 1}^{1 / 2} B_{i, 1}^{\delta_{m s}}
\end{array}\right]=: B_{i, 1}
$$


where

$$
\begin{aligned}
B_{1,1}^{\delta_{m}}:=(1 / 2)\left[\int_{0}^{1} V_{\bar{\alpha}_{1,1}} d r, \int_{0}^{1} V_{\bar{\alpha}_{1,3}} d r\right]^{\prime} \\
B_{2,1}^{\delta_{m}}:=\left[\int_{0}^{1} V_{\bar{\alpha}_{2}} d r\right] \\
B_{1,1}^{\delta_{m s}}:=(1 / 2)\left[\int_{0}^{1} V_{\bar{\alpha}_{1,1}} d r-\int_{0}^{\lambda} V_{\bar{\alpha}_{1,1}} d r, \int_{0}^{1} V_{\bar{\alpha}_{1,3}} d r-\int_{0}^{\lambda} V_{\bar{\alpha}_{1,3}} d r\right]^{\prime} \\
B_{2,1}^{\delta_{m s}}:=\left[\int_{0}^{1} V_{\bar{\alpha}_{2}} d r-\int_{0}^{\lambda} V_{\bar{\alpha}_{2}} d r\right] .
\end{aligned}
$$

The proof of this Lemma follows directly from Lemma S.1.

Lemma S.5. Let the conditions of Theorem S.7 hold. Suppose that regression (3.6) is estimated by using $T_{b} \in \Lambda^{*}$ and $T_{b 0}$ is the true break date. Then as $T \rightarrow \infty$, if the data are $I_{\omega_{i}}(0)$

$$
T^{1 / 2} \hat{\delta}_{\omega_{i}, m s} \stackrel{d}{\rightarrow} \Omega_{i, 0}^{1 / 2}\left[P_{i, 0}^{\delta_{m s}}(\lambda)+\Psi_{i}^{\delta_{m s}}\left(\lambda, \lambda_{0}\right) \kappa_{\omega_{i}, m s}\right]
$$

while if the data are $I_{\omega_{i}}(1)$

$$
T^{-1 / 2} \hat{\delta}_{\omega_{i}, m s} \stackrel{d}{\rightarrow} \Omega_{i, 1}^{1 / 2}\left[P_{i, 1}^{\delta_{m s}}(\lambda)+\Psi_{i}^{\delta_{m s}}\left(\lambda, \lambda_{0}\right) \kappa_{\omega_{i}, m s}\right]
$$

Proof. Using the familiar Frisch-Waugh Theorem, the OLS estimate of $\delta_{\omega_{i}, m s}$ under the alternative hypothesis is given by

$$
\begin{aligned}
T^{1 / 2} \hat{\delta}_{\omega_{i}, m s} & =\left[\sum_{t=1}^{T} \tilde{F}_{i, m s}(t, \lambda) \tilde{F}_{i, m s}(t, \lambda)^{\prime}\right]^{-1}\left[\sum_{t=1}^{T} \tilde{F}_{i, m s}(t, \lambda) \tilde{F}_{i, m s}\left(t, \lambda_{0}\right)^{\prime}\right] \delta_{\omega_{i}, m s} \\
& +\left[\sum_{t=1}^{T} \tilde{F}_{i, m s}(t, \lambda) \tilde{F}_{i, m s}(t, \lambda)^{\prime}\right]^{-1} \sum_{t=1}^{T} \tilde{F}_{i, m s}(t, \lambda) u_{i, t} .
\end{aligned}
$$

When $u_{i, t}$ is $I_{\omega_{i}}(0)$ we have

$$
\begin{aligned}
T^{1 / 2} \hat{\delta}_{\omega_{i}, m s} & =\left[T^{-1} \sum_{t=1}^{T} \tilde{F}_{i, m s}(t, \lambda) \tilde{F}_{i, m s}(t, \lambda)^{\prime}\right]^{-1}\left[T^{-1} \sum_{t=1}^{T} \tilde{F}_{i, m s}(t, \lambda) \tilde{F}_{i, m s}\left(t, \lambda_{0}\right)^{\prime}\right] T^{1 / 2} \delta_{\omega_{i}, m s} \\
& +\left[T^{-1} \sum_{t=1}^{T} \tilde{F}_{i, m s}(t, \lambda) \tilde{F}_{i, m s}(t, \lambda)^{\prime}\right]^{-1} T^{-1 / 2} \sum_{t=1}^{T} \tilde{F}_{i, m s}(t, \lambda) u_{i, t} .
\end{aligned}
$$

The first term is given by

$$
\begin{aligned}
& {\left[T^{-1} \sum_{t=1}^{T} \tilde{F}_{i, m s}(t, \lambda) \tilde{F}_{i, m s}(t, \lambda)^{\prime}\right]^{-1}\left[T^{-1} \sum_{t=1}^{T} \tilde{F}_{i, m s}(t, \lambda) \tilde{F}_{i, m s}\left(t, \lambda_{0}\right)^{\prime}\right] T^{1 / 2} \delta_{\omega_{i}, m s} } \\
& \stackrel{d}{\rightarrow} \Omega_{i, 0}^{1 / 2} \Psi_{i}^{\delta_{m s}}\left(\lambda, \lambda_{0}\right) \kappa_{\omega_{i}, m s}
\end{aligned}
$$


and the second term is asymptotically equivalent to

$$
R_{i}\left[T^{-1} \sum_{t=1}^{T} F_{i}(t, \lambda) F_{i}(t, \lambda)^{\prime}\right]^{-1} T^{-1 / 2} \sum_{t=1}^{T} F_{i}(t, \lambda) u_{i, t} .
$$

Using Lemma S.4 we have that

$$
\begin{aligned}
R_{i}\left[T^{-1} \sum_{t=1}^{T} F_{i}(t, \lambda) F_{i}(t, \lambda)^{\prime}\right]^{-1} T^{-1 / 2} \sum_{t=1}^{T} F_{i}(t, \lambda) u_{i, t} \stackrel{d}{\rightarrow} R_{i}\left[\int_{0}^{1} F_{i}(r, \lambda) F_{i}(r, \lambda)^{\prime} d r\right]^{-1} B_{i, 0} \\
=: \Omega_{i, 0}^{1 / 2} P_{i, 0}^{\delta_{m s}}(\lambda) .
\end{aligned}
$$

Combining these results gives us $T^{1 / 2} \hat{\delta}_{\omega_{i}, m s} \stackrel{d}{\rightarrow} \Omega_{i, 0}^{1 / 2}\left[P_{i, 0}^{\delta_{m s}}(\lambda)+\Psi_{i}^{\delta_{m s}}\left(\lambda, \lambda_{0}\right) \kappa_{\omega_{i}, m s}\right]$

When the data are $I_{\omega_{i}}(1)$ we have that

$$
\begin{aligned}
T^{-1 / 2} \hat{\delta}_{\omega_{i}, m s} & =\left[T^{-1} \sum_{t=1}^{T} \tilde{F}_{i, m s}(t, \lambda) \tilde{F}_{i, m s}(t, \lambda)^{\prime}\right]^{-1}\left[T^{-1} \sum_{t=1}^{T} \tilde{F}_{i, m s}(t, \lambda) \tilde{F}_{i, m s}\left(t, \lambda_{0}\right)\right] T^{-1 / 2} \delta_{\omega_{i}, m s} \\
& +\left[T^{-1} \sum_{t=1}^{T} \tilde{F}_{i, m s}(t, \lambda) \tilde{F}_{i, m s}(t, \lambda)^{\prime}\right]^{-1} T^{-1} \sum_{t=1}^{T} T^{-1 / 2} \tilde{F}_{i, m s}(t, \lambda) u_{i, t} .
\end{aligned}
$$

The first term is given by

$$
\begin{aligned}
& {\left[T^{-1} \sum_{t=1}^{T} \tilde{F}_{i, m s}(t, \lambda) \tilde{F}_{i, m s}(t, \lambda)^{\prime}\right]^{-1}\left[T^{-1} \sum_{t=1}^{T} \tilde{F}_{i, m s}(t, \lambda) \tilde{F}_{i, m s}\left(t, \lambda_{0}\right)^{\prime}\right] T^{-1 / 2} \delta_{\omega_{i}, m s} } \\
\stackrel{d}{\rightarrow} & \Omega_{i, 1}^{1 / 2} \Psi_{i}^{\delta_{m s}}\left(\lambda, \lambda_{0}\right) \kappa_{\omega_{i}, m s}
\end{aligned}
$$

and the second term is asymptotically equivalent to

$$
R_{i}\left[T^{-1} \sum_{t=1}^{T} F_{i}(t, \lambda) F_{i}(t, \lambda)^{\prime}\right]^{-1} T^{-1} \sum_{t=1}^{T} T^{-1 / 2} F_{i}(t, \lambda) u_{i, t} .
$$

Using the result from Lemma S.4 we, thus, have that

$$
\begin{aligned}
R_{i}\left[T^{-1} \sum_{t=1}^{T} F_{i}(t, \lambda) F_{i}(t, \lambda)^{\prime}\right]^{-1} T^{-1} \sum_{t=1}^{T} T^{-1 / 2} F_{i}(t, \lambda) u_{i, t} & \stackrel{d}{\rightarrow} R_{i}\left[\int_{0}^{1} F_{i}(r, \lambda) F_{i}(r, \lambda)^{\prime} d r\right]^{-1} B_{i, 1} \\
& =: \Omega_{i, 1}^{1 / 2} P_{i, 1}^{\delta_{m s}}(\lambda) .
\end{aligned}
$$

Combining these results gives us $T^{-1 / 2} \hat{\delta}_{\omega_{i}, m s} \stackrel{d}{\rightarrow} \Omega_{i, 1}^{1 / 2}\left[P_{i, 1}^{\delta_{m s}}(\lambda)+\Psi_{i}^{\delta_{m s}}\left(\lambda, \lambda_{0}\right) \kappa_{\omega_{i}, m s}\right]$

Lemma S.6. Let the conditions of Theorem S.7 hold. Suppose that regression (3.6) is estimated by using $T_{b} \in \Lambda^{*}$ and $T_{b 0}$ is the true break date. Then as $T \rightarrow \infty$, if the data are $I_{\omega_{i}}(0)$

$T^{-1 / 2} \hat{S}_{i,\lfloor r T\rfloor} \stackrel{d}{\rightarrow} \Omega_{i, 0}^{1 / 2}\left[Q_{i, 0}^{\delta_{m s}}(r, \lambda)-\Delta_{i}\left(r, \lambda, \lambda_{0}, \kappa_{\omega_{i}, m s}\right)\right]:=\Omega_{i, 0}^{1 / 2} Q_{\kappa_{\omega_{i}, m s}, i, 0}^{\delta_{m s}}\left(r, \lambda, \lambda_{0}, \kappa_{\omega_{i}, m s}\right)$

while if the data are $I_{\omega_{i}}(1)$ then

$T^{-3 / 2} \hat{S}_{i,\lfloor r T\rfloor} \stackrel{d}{\rightarrow} \Omega_{i, 1}^{1 / 2}\left[Q_{i, 1}^{\delta_{m s}}(r, \lambda)-\Delta_{i}\left(r, \lambda, \lambda_{0}, \kappa_{\omega_{i}, m s}\right)\right]:=\Omega_{i, 1}^{1 / 2} Q_{\kappa_{\omega_{i}, m s}, i, 1}^{\delta_{i_{s}}}\left(r, \lambda, \lambda_{0}, \kappa_{\omega_{i}, m s}\right)$ 
Proof. Under the alternative hypothesis we have that $\hat{u}_{i, t}=u_{i, t}-F_{i, m s}(t, \lambda)^{\prime} \hat{\delta}_{\omega_{i}, m s}+$ $F_{i, m s}\left(t, \lambda_{0}\right)^{\prime} \delta_{\omega_{i}, m s}$, so that $z_{i, t} \hat{u}_{i, t}=z_{i, t} u_{i, t}-\breve{F}_{i, m s}(t, \lambda)^{\prime} \hat{\delta}_{\omega_{i}, m s}+\breve{F}_{i, m s}\left(t, \lambda_{0}\right)^{\prime} \delta_{\omega_{i}, m s}$. Summing yields

$$
\hat{S}_{i,\lfloor r T\rfloor}=\sum_{t=1}^{\lfloor r T\rfloor} z_{i, t} u_{i, t}-\sum_{t=1}^{\lfloor r T\rfloor} \breve{F}_{i, m s}(t, \lambda)^{\prime} \hat{\delta}_{\omega_{i}, m s}+\sum_{t=1}^{\lfloor r T\rfloor} \breve{F}_{i, m s}\left(t, \lambda_{0}\right)^{\prime} \delta_{\omega_{i}, m s} .
$$

When the date are $I_{\omega_{i}}(0)$ we have

$$
\begin{aligned}
& T^{-1 / 2} \hat{S}_{i,\lfloor r T\rfloor} \\
= & T^{-1 / 2} \sum_{t=1}^{\lfloor r T\rfloor} z_{i, t} u_{i, t}-T^{-1} \sum_{t=1}^{\lfloor r T\rfloor} \breve{F}_{i, m s}(t, \lambda)^{\prime} T^{1 / 2} \hat{\delta}_{\omega_{i}, m s}+T^{-1} \sum_{t=1}^{\lfloor r T\rfloor} \breve{F}_{i, m s}\left(t, \lambda_{0}\right)^{\prime} T^{1 / 2} \delta_{\omega_{i}, m s} \\
\stackrel{d}{\rightarrow} & \Omega_{i, 0}^{1 / 2}\left[W^{i}(r)-\left[\int_{0}^{r} \breve{F}_{i, m s}(s, \lambda)^{\prime} d s\right]\left[P_{i, 0}^{\delta_{m s}}(\lambda)+\Psi_{i}^{\delta_{m s}}\left(\lambda, \lambda_{0}\right) \kappa_{\omega_{i}, m s}\right]+\left[\int_{0}^{r} \breve{F}_{i, m s}\left(s, \lambda_{0}\right)^{\prime} d s\right] \kappa_{\omega_{i}, m s}\right] \\
= & \Omega_{i, 0}^{1 / 2}\left[\left(W^{i}(r)-\left[\int_{0}^{r} \breve{F}_{i, m s}(s, \lambda)^{\prime} d s\right] P_{i, 0}^{\delta_{m s}}(\lambda)\right)\right. \\
- & \left.\left(\left\{\left[\int_{0}^{r} \breve{F}_{i, m s}(s, \lambda)^{\prime} d s\right] \Psi_{i}^{\delta_{m s}}\left(\lambda, \lambda_{0}\right)-\left[\int_{0}^{r} \breve{F}_{i, m s}\left(s, \lambda_{0}\right)^{\prime} d s\right]\right\} \kappa_{\omega_{i}, m s}\right)\right] \\
= & \Omega_{i, 0}^{1 / 2}\left[Q_{i, 0}^{\delta_{m s}}(r, \lambda)-\Delta_{i}\left(r, \lambda, \lambda_{0}, \kappa_{\omega_{i}, m s}\right)\right]=: \Omega_{i, 0}^{1 / 2} Q_{\kappa_{\omega_{i}, m s}, i, 0}^{\delta_{m s}}\left(r, \lambda, \lambda_{0}, \kappa_{\omega_{i}, m s}\right)
\end{aligned}
$$

from Lemmas S.1 and S.5.

When the data are $I_{\omega_{i}}(1)$ we have

$$
\begin{aligned}
& T^{-3 / 2} \hat{S}_{i,\lfloor r T\rfloor} \\
= & T^{-1} \sum_{t=1}^{\lfloor r T\rfloor} T^{-1 / 2} z_{i, t} u_{i, t}-T^{-1} \sum_{t=1}^{\lfloor r T\rfloor} \breve{F}_{i, m s}(t, \lambda)^{\prime} T^{-1 / 2} \hat{\delta}_{\omega_{i}, m s}+T^{-1} \sum_{t=1}^{\lfloor r T\rfloor} \breve{F}_{i, m s}\left(t, \lambda_{0}\right)^{\prime} T^{-1 / 2} \delta_{\omega_{i}, m s} \\
\stackrel{d}{\rightarrow} & \Omega_{i, 1}^{1 / 2}\left[V^{i}(r)-\left[\int_{0}^{r} \breve{F}_{i, m s}(s, \lambda)^{\prime} d s\right]\left[P_{i, 1}^{\delta_{m s}}(\lambda)+\Psi_{i}^{\delta_{m s}}\left(\lambda, \lambda_{0}\right) \kappa_{\omega_{i}, m s}\right]+\left[\int_{0}^{r} \breve{F}_{i, m s}\left(s, \lambda_{0}\right)^{\prime} d s\right] \kappa_{\omega_{i}, m s}\right] \\
= & \Omega_{i, 1}^{1 / 2}\left[\left(V^{i}(r)-\left[\int_{0}^{r} \breve{F}_{i, m s}(s, \lambda)^{\prime} d s\right] P_{i, 1}^{\delta_{m s}}(\lambda)\right)\right. \\
- & \left.\left(\left\{\left[\int_{0}^{r} \breve{F}_{i, m s}(s, \lambda)^{\prime} d s\right] \Psi_{i}^{\delta_{m s}}\left(\lambda, \lambda_{0}\right)-\left[\int_{0}^{r} \breve{F}_{i, m s}\left(s, \lambda_{0}\right)^{\prime} d s\right]\right\} \kappa_{\omega_{i}, m s}\right)\right] \\
= & \Omega_{i, 1}^{1 / 2}\left[Q_{i, 1}^{\delta_{m s}}(r, \lambda)-\Delta_{i}\left(r, \lambda, \lambda_{0}, \kappa_{\omega_{i}, m s}\right)\right]=: \Omega_{i, 1}^{1 / 2} Q_{\kappa_{\omega_{i}, m s}, i, 1}^{\delta_{m s}}\left(r, \lambda, \lambda_{0}, \kappa_{\omega_{i}, m s}\right)
\end{aligned}
$$

from Lemmas S.1 and S.5.

Lemma S.7. Let the conditions of Theorem S.7 hold. Suppose that regression (3.6) is estimated by using $T_{b} \in \Lambda^{*}$ and $T_{b 0}$ is the true break date. Then as $T \rightarrow \infty$ if the data are $I_{\omega_{i}}(1)$

$$
T^{-1 / 2} z_{i,\lfloor r T\rfloor} \hat{u}_{i,\lfloor r T\rfloor} \stackrel{d}{\rightarrow} \Omega_{i, 1}^{1 / 2}\left(\hat{V}_{\bar{\alpha}_{i}}^{i}(r, \lambda)+\breve{F}_{i, m s}(r, \lambda)^{\prime}\left[I-\Psi_{i}^{\delta_{m s}}\left(\lambda, \lambda_{0}\right)\right] \kappa_{\omega_{i}, m s}\right) .
$$


Proof. Under the alternative hypothesis when we have that

$$
\hat{u}_{i, t}=u_{i, t}-F_{i, m s}(t, \lambda)^{\prime} \hat{\delta}_{\omega_{i}, m s}+F_{i, m s}\left(t, \lambda_{0}\right)^{\prime} \delta_{\omega_{i}, m s},
$$

so that when the data are $I_{\omega_{i}}(1)$

$$
\begin{aligned}
T^{-1 / 2} & z_{i,\lfloor r T\rfloor} \hat{u}_{i,\lfloor r T\rfloor}=T^{-1 / 2} z_{i,\lfloor r T\rfloor} u_{i,\lfloor r T\rfloor}-\breve{F}_{i, m s}(\lfloor r T\rfloor, \lambda)^{\prime} T^{-1 / 2} \hat{\delta}_{\omega_{i}, m s}+\breve{F}_{i, m s}\left(\lfloor r T\rfloor, \lambda_{0}\right)^{\prime} T^{-1 / 2} \delta_{\omega_{i}, m s} \\
\stackrel{d}{\rightarrow} & \Omega_{i, 1}^{1 / 2}\left[V^{i}(r)-\breve{F}_{i, m s}(r, \lambda)^{\prime}\left[P_{i, 1}^{\delta_{m s}}(\lambda)+\Psi_{i}^{\delta_{m s}}\left(\lambda, \lambda_{0}\right) \kappa_{\omega_{i}, m s}\right]+\breve{F}_{i, m s}\left(r, \lambda_{0}\right)^{\prime} \kappa_{\omega_{i}, m s}\right] \\
= & \Omega_{i, 1}^{1 / 2}\left[\left(V^{i}(r)-\breve{F}_{i, m s}(r, \lambda)^{\prime} P_{i, 1}^{\delta_{m s}}(\lambda)\right)-\breve{F}_{i, m s}(r, \lambda)^{\prime} \Psi_{i}^{\delta_{m s}}\left(\lambda, \lambda_{0}\right)_{i, 2}+\breve{F}_{i, m s}\left(r, \lambda_{0}\right)^{\prime} \kappa_{\omega_{i}, m s}\right] \\
=: & \Omega_{i, 1}^{1 / 2}\left[\hat{V}^{i}(r, \lambda)+\breve{F}_{i, m s}(r, \lambda)^{\prime}\left[I-\Psi_{i}^{\delta_{m s}}\left(\lambda, \lambda_{0}\right)\right] \kappa_{\omega_{i}, m s}\right]
\end{aligned}
$$

from Lemmas S.1 and S.5.

Lemma S.8. Let the conditions of Theorem S.7 hold. Suppose that regression (3.6) is estimated by using $T_{b} \in \Lambda^{*}$ and $T_{b 0}$ is the true break date. Then letting the bandwidth parameter be a fixed proportion of the sample size, $M=\lfloor b T\rfloor, b \in(0,1]$, then as $T \rightarrow \infty$ if the data are $I_{\omega_{i}}(0)$

$$
\begin{aligned}
\hat{\sigma}_{V, \omega_{i}}^{2} \stackrel{d}{\rightarrow} \Omega_{i, 0} \int_{0}^{1} Q_{\kappa_{\omega_{i}, m s}, i, 0}^{\delta_{m s}}\left(r, \lambda, \lambda_{0}, \kappa_{\omega_{i}, m s}\right) Q_{\kappa_{\omega_{i}, m s}, i, 0}^{\delta_{m s}}\left(r, \lambda, \lambda_{0}, \kappa_{\omega_{i}, m s}\right)^{\prime} d r \\
\hat{\sigma}_{B V, \omega_{i}}^{2} \stackrel{d}{\rightarrow} \Omega_{i, 0} \Phi_{\kappa_{\omega_{i}, m s}, i, 0}\left(b, k^{*}(\cdot), \lambda, \lambda_{0}, \kappa_{\omega_{i}, m s}\right)
\end{aligned}
$$

and if the data are $I_{\omega_{i}}(1)$ then

$$
\begin{aligned}
T^{-2} \hat{\sigma}_{V, \omega_{i}}^{2} \stackrel{d}{\rightarrow} \Omega_{i, 1} \int_{0}^{1} Q_{\kappa_{\omega_{i}, m s}, i, 1}^{\delta_{m s}}\left(r, \lambda, \lambda_{0}, \kappa_{\omega_{i}, m s}\right) Q_{\kappa_{\omega_{i}, m s}, i, 1}^{\delta_{m s}}\left(r, \lambda, \lambda_{0}, \kappa_{\omega_{i}, m s}\right)^{\prime} d r \\
T^{-2} \hat{\sigma}_{B V, \omega_{i}}^{2} \stackrel{d}{\rightarrow} \Omega_{i, 1} \Phi_{\kappa_{\omega_{i}, m s}, i, 1}\left(b, k^{*}(\cdot), \lambda, \lambda_{0}, \kappa_{\omega_{i}, m s}\right)
\end{aligned}
$$

Proof. The proof of this Lemma follows directly from the CMT using Lemma S.6 and Theorem 1 of Bunzel and Vogelsang (2005).

\section{Proof of Theorem S.6:}

The proof of part (a) of this Theorem when the data are $I_{\omega_{1}}(0)$ can be obtained with trivial modification to the part (b) of the theorem. When the data are $I_{\omega_{1}}(1)$ we have for any $T_{b} \in \Lambda^{*}$

$$
V R_{\delta_{\omega_{1}, m s}}\left(T_{b}\right)=T^{-3} \operatorname{trace}\left[\hat{\Gamma}_{1}^{-1} \sum_{t=3}^{T}\left(\sum_{j=1}^{t} z_{1, j} \hat{u}_{1, j}\right)\left(\sum_{j=1}^{t} z_{1, j} \hat{u}_{1, j}\right)^{\prime}\right]
$$


where $\hat{\Gamma}_{1}:=T^{-1} \sum_{t=f_{1}+1}^{T}\left(\hat{u}_{1, t} z_{1, t}\right)\left(\hat{u}_{1, t} z_{1, t}\right)^{\prime}$. Defining $\hat{S}_{1, t}:=\sum_{i=1}^{t} z_{1, s} \hat{u}_{1, s}$ we have that

$$
\begin{aligned}
V R_{\delta_{\omega_{1}, m s}}\left(T_{b}\right) & =\operatorname{trace}\left[T^{-2} \sum_{t=1}^{T}\left(\hat{u}_{1, t} z_{1, t}\right)\left(\hat{u}_{1, t} z_{1, t}\right)^{\prime}\right]^{-1} \times\left[T^{-4} \sum_{t=1}^{T} \hat{S}_{1, t} \hat{S}_{1, t}^{\prime}\right] \\
& =\operatorname{trace}\left[T^{-1} \sum_{t=1}^{T} T^{-1 / 2}\left(\hat{u}_{1, t} z_{1, t}\right) T^{-1 / 2}\left(\hat{u}_{1, t} z_{1, t}\right)^{\prime}\right]^{-1} \times\left[T^{-1} \sum_{t=1}^{T} T^{-3 / 2} \hat{S}_{1, t} T^{-3 / 2} \hat{S}_{1, t}^{\prime}\right] \\
& \stackrel{d}{\rightarrow} \operatorname{trace}\left[\int_{0}^{1}\left(\hat{V}^{1}(r, \lambda)+\check{F}_{1,2}(r, \lambda)^{\prime}\left[I-\Psi_{1}^{\delta_{m s}}\left(\lambda, \lambda_{0}\right)\right] \kappa_{\omega_{1}, m s}\right)\right. \\
& \left.\times\left(\hat{V}^{1}(r, \lambda)+\check{F}_{1,2}(r, \lambda)^{\prime}\left[I-\Psi_{1}^{\delta_{m s}}\left(\lambda, \lambda_{0}\right)\right] \kappa_{\omega_{1}, m s}\right)^{\prime} d r\right]^{-1} \\
& \times \int_{0}^{1} Q_{\kappa_{\omega_{1}, m s}, 1,1}^{\delta_{m s}}\left(r, \lambda, \lambda_{0}, \kappa_{\omega_{1}, m s}\right) Q_{\kappa_{\omega_{1}, m s}, 1,1}^{\delta_{m s}}\left(r, \lambda, \lambda_{0}, \kappa_{\omega_{1}, m s}\right)^{\prime} d r
\end{aligned}
$$

by Lemma S.6 and Lemma S.7 and the CMT. The proof of the Theorem then follows directly from this result and the CMT. The proof for the Nyquist frequency unit root test statistic follows in a similar manner to that of the annual frequency unit root test statistic.

\section{Proof of Theorem S.7:}

(a) When the data are $I_{\omega_{1}}(0)$ we have that

$$
\begin{aligned}
W_{\omega_{1}, m s, V}\left(T_{b}\right)= & \left(T^{1 / 2} \hat{\delta}_{\omega_{1}, m s}\right)^{\prime}\left[\hat{\sigma}_{V, \omega_{1}}^{2}\left[T^{-1} \sum_{t=1}^{T} \tilde{F}_{1,2}(t, \lambda) \tilde{F}_{1,2}(t, \lambda)^{\prime}\right]^{-1}\right]^{-1}\left(T^{1 / 2} \hat{\delta}_{\omega_{1}, m s}\right) \\
\stackrel{d}{\rightarrow}\left[P_{1,0}^{\delta_{m s}}(\lambda)+\Psi_{1}^{\delta_{m s}}\left(\lambda, \lambda_{0}\right) \kappa_{\omega_{1}, m s}\right]^{\prime} \times & {\left[\int_{0}^{1} Q_{\kappa_{\omega_{1}, m s}, 1,1}^{\delta_{m s}}\left(r, \lambda, \lambda_{0}, \kappa_{\omega_{1}, m s}\right) Q_{\kappa_{\omega_{1}, m s}, 1,1}^{\delta_{m_{1}}}\left(r, \lambda, \lambda_{0}, \kappa_{\omega_{1}, m s}\right)^{\prime} d r\right.} \\
\times & {\left.\left[\int_{0}^{1} \tilde{F}_{1,2}(r, \lambda) \tilde{F}_{1,2}(r, \lambda)^{\prime} d r\right]^{-1}\right]^{-1} } \\
& \times\left[P_{1,0}^{\delta_{m s}}(\lambda)+\Psi_{1}^{\delta_{m s}}\left(\lambda, \lambda_{0}\right) \kappa_{\omega_{1}, m s}\right]
\end{aligned}
$$

by Lemmas S.8 and S.5 and the CMT. 


$$
\begin{aligned}
W_{\omega_{1}, m s, B V}\left(T_{b}\right)= & \left(T^{1 / 2} \hat{\delta}_{\omega_{1}, m s}\right)^{\prime}\left[\hat{\sigma}_{B V, \omega_{1}}^{2}\left[T^{-1} \sum_{t=1}^{T} \tilde{F}_{1,2}(t, \lambda) \tilde{F}_{1,2}(t, \lambda)^{\prime}\right]^{-1}\right]^{-1}\left(T^{1 / 2} \hat{\delta}_{\omega_{1}, m s}\right) \\
\stackrel{d}{\rightarrow} & {\left[P_{1,0}^{\delta_{m s}}(\lambda)+\Psi_{1}^{\delta_{m s}}\left(\lambda, \lambda_{0}\right) \kappa_{\omega_{1}, m s}\right]^{\prime}\left[\Phi_{\kappa_{\omega_{1}, m s}, 1,0}\left(b, k^{*}(\cdot), \lambda, \lambda_{0}, \kappa_{\omega_{1}, m s}\right)\right.} \\
& \left.\times\left[\int_{0}^{1} \tilde{F}_{1,2}(r, \lambda) \tilde{F}_{1,2}(r, \lambda)^{\prime} d r\right]^{-1}\right]^{-1} \\
& \times\left[P_{1,0}^{\delta_{m s}}(\lambda)+\Psi_{1}^{\delta_{m s}}\left(\lambda, \lambda_{0}\right) \kappa_{\omega_{1}, m s}\right]
\end{aligned}
$$

by Lemmas S.8 and S.5 and the CMT. The proof of part (b) of this theorem follows from part (a) and the CMT

\section{Proof of Theorem S.8:}

(a) If the data are $I_{\omega_{1}}(1)$

$$
\begin{aligned}
W_{\omega_{1}, m s, V}\left(T_{b}\right) & =\left(T^{-1 / 2} \hat{\delta}_{\omega_{1}, m s}\right)^{\prime}\left[T^{-2} \hat{\sigma}_{V, \omega_{1}}^{2}\left[T^{-1} \sum_{t=1}^{T} \tilde{F}_{1,2}(t, \lambda) \tilde{F}_{1,2}(t, \lambda)^{\prime}\right]^{-1}\right]^{-1}\left(T^{-1 / 2} \hat{\delta}_{\omega_{1}, m s}\right) \\
& \stackrel{d}{\rightarrow}\left[P_{1,1}^{\delta_{m s}}(\lambda)+\Psi_{1}^{\delta_{m s}}\left(\lambda, \lambda_{0}\right) \kappa_{\omega_{1}, m s}\right]^{\prime} \\
& \times\left[\int_{0}^{1} Q_{\kappa_{\omega_{1}, m s}, 1,1}^{\delta_{m s}}\left(r, \lambda, \lambda_{0}, \kappa_{\omega_{1}, m s}\right) Q_{\kappa_{\omega_{1}, m s}, i, 1}^{\delta_{m s}}\left(r, \lambda, \lambda_{0}, \kappa_{\omega_{1}, m s}\right)^{\prime} d r\right. \\
& \left.\times\left[\int_{0}^{1} \tilde{F}_{1,2}(r, \lambda) \tilde{F}_{1,2}(r, \lambda)^{\prime} d r\right]^{-1}\right]^{-1} \\
& \times\left[P_{1,1}^{\delta_{m s}}(\lambda)+\Psi_{1}^{\delta_{m s}}\left(\lambda, \lambda_{0}\right) \kappa_{\omega_{1}, m s}\right]
\end{aligned}
$$

by Lemmas S.8 and S.5 and the CMT.

$$
\begin{aligned}
W_{\omega_{1}, m s, B V}\left(T_{b}\right) & =\left(T^{-1 / 2} \hat{\delta}_{\omega_{1}, m s}\right)^{\prime}\left[T^{-2} \hat{\sigma}_{B V, \omega_{1}}^{2}\left[T^{-1} \sum_{t=1}^{T} \tilde{F}_{1,2}(t, \lambda) \tilde{F}_{1,2}(t, \lambda)^{\prime}\right]^{-1}\right]^{-1}\left(T^{-1 / 2} \hat{\delta}_{\omega_{1}, m s}\right) \\
& \stackrel{d}{\rightarrow}\left[P_{1,1}^{\delta_{m s}}(\lambda)+\Psi_{1}^{\delta_{m s}}\left(\lambda, \lambda_{0}\right) \kappa_{\omega_{1}, m s}\right]^{\prime}\left[\Phi_{\kappa_{\omega_{1}, m s}, i, 1}\left(b, k^{*}(\cdot), \lambda, \lambda_{0}, \kappa_{\omega_{1}, m s}\right)\right. \\
& \left.\times\left[\int_{0}^{1} \tilde{F}_{1,2}(r, \lambda) \tilde{F}_{1,2}(r, \lambda)^{\prime} d r\right]^{-1}\right]^{-1} \\
& \times\left[P_{1,1}^{\delta_{m s}}(\lambda)+\Psi_{1}^{\delta_{m s}}\left(\lambda, \lambda_{0}\right) \kappa_{\omega_{1}, m s}\right]
\end{aligned}
$$

by Lemmas S.8 and S.5 and the CMT. The proof of part (b) of this theorem follows from part (a) and the CMT. The proof of Theorems S.9 and S.10 follow directly from the proofs of Theorems S.7 and S.8, respectively. 


\section{ADDITIONAL REFERENCES}

These are the additional references cited in this supplement and not listed in the main paper.

del Barrio Castro, T., Osborn, D.R., and Taylor A.M.R. (2012). On Augmented HEGY Tests for Seasonal Unit Roots. Econometric Theory 28, 1121-1143.

Schwarz, G. (1978). Estimating the dimension of a model. Annals of Statistics 6, 461464. 